\title{
RADIO OBSERVATIONS OF THE HUBBLE DEEP FIELD-SOUTH REGION. IV. OPTICAL PROPERTIES OF THE FAINT RADIO POPULATION
}

\author{
Minh T. Huynh ${ }^{1}$, Carole A. JaCkson ${ }^{2}$, Ray P. Norris ${ }^{2}$, and Alberto Fernandez-Soto ${ }^{3}$ \\ ${ }^{1}$ Spitzer Science Center, MS220-6, California Institute of Technology, Pasadena, CA 91125, USA; mhuynh@ipac.caltech.edu \\ ${ }^{2}$ Australia Telescope National Facility, CSIRO Radiophysics Laboratory, P.O. Box 76, Epping, NSW 2121, Australia \\ ${ }^{3}$ Grupo de Radioastronomía, Dept. Astronomia, Universitat de Valencia. Av. Dr. Moliner 50, Burjassot, 46100-Valencia, Spain \\ Received 2007 October 19; accepted 2008 March 21; published 2008 May 15
}

\begin{abstract}
The Australia Telescope Hubble Deep Field-South (ATHDF-S) survey of the Hubble Deep Field-South (HDF-S) reaches sensitivities of $\sim 10 \mu \mathrm{Jy}$ at $1.4,2.5,5.2$, and $8.7 \mathrm{GHz}$, making the ATHDF-S one of the deepest surveys ever performed with the Australia Telescope Compact Array (ATCA). Here, we present the optical identifications of the ATHDF-S radio sources using data from the literature. We find that $\sim 66 \%$ of the radio sources have optical counterparts to $I=23.5 \mathrm{mag}$. Deep Hubble Space Telescope (HST) imaging of the area identifies a further $12 \%$ of radio sources. We present new spectroscopic observations for 98 of the radio sources and supplement these spectroscopic redshifts with photometric ones calculated from five-band optical imaging. The host galaxy colors and radio-to-optical ratios indicate that low-luminosity (or "radio-quiet") active galactic nuclei make up a significant proportion of the sub-mJy radio population, a result which is in accordance with a number of other deep radio studies. The radio-to-optical ratios of the bright $\left(S_{1.4 \mathrm{GHz}}>1 \mathrm{mJy}\right)$ sources are consistent with a bimodal distribution.
\end{abstract}

Key words: radio continuum: galaxies - surveys

Online-only material: color figures, machine-readable tables

\section{INTRODUCTION}

Many massive galaxies in the local universe harbor a supermassive black hole, and this implies that most, if not all, galaxies hosted an active galactic nucleus (AGN) at some point in time (Kormendy \& Richstone 1995). It is now thought that star formation and AGNs are linked by some mechanism collectively known as "AGN-driven feedback" (e.g. Croton et al. 2006). Radio emission can be produced by both AGN and star-forming processes, and thus radio surveys provide a unique window to study the cosmic evolution of both these important processes.

Early radio surveys $\left(S_{1.4 \mathrm{GHz}} \gtrsim 100 \mathrm{mJy}\right.$ ) found objects with powerful radio emitting jets, which are generally termed "radioloud" AGNs. Recent deep radio surveys have found that the Euclidean-normalized radio source counts flatten below about $1 \mathrm{mJy}$ and this cannot be explained by a population of radioloud AGNs. A nonevolving population of local $(z<0.1)$ lowluminosity radio galaxies (Wall et al. 1986), strongly evolving normal spirals (Condon 1984, 1989), and starburst galaxies (Windhorst et al. 1985; Rowan-Robinson et al. 1993) have all been suggested to explain this new population. The faint radio source counts have been successfully modeled by star-forming galaxies (Seymour et al. 2004; Huynh et al. 2005). However, it has also been shown that low-luminosity radio-quiet AGNs can also contribute to the flattening of the source count at sub-mJy levels (Jarvis \& Rawlings 2004).

It is now clear that the sub-mJy population is made up of both star-forming galaxies and low-luminosity AGNs, but surprisingly little is known about the exact mix. This is because the optical counterparts to the radio sources are faint and hence spectroscopic follow-up is difficult and/or requires massive amounts of telescope time. Pioneering work in the Hubble Deep FieldNorth (HDF-N) by Richards et al. (1999) found that roughly $60 \%$ of sub-mJy $\left(S_{1.4 \mathrm{GHz}}>0.04 \mathrm{mJy}\right)$ sources are associated with bright $(I \sim 22)$ disk galaxies, $20 \%$ are low-luminosity AGNs, and the remaining $20 \%$ have no counterpart brighter than $I=25$. Deep Advanced Camera for Surveys (ACS) imaging $\left(i_{775}<27\right)$ of the Great Observatories Origins Deep Survey (GOODS) South field identified optical counterparts to $90 \%$ of the faint $\left(S_{1.4 \mathrm{GHz}}>0.06 \mathrm{mJy}\right)$ radio sources, and about $60 \%$ of the GOODS radio sources with spectroscopic information have a spectrum consistent with star-formation processes (Afonso et al. 2006). Simpson et al. (2006) find that $90 \%$ of $S_{1.4 \mathrm{GHz}}>$ $0.1 \mathrm{mJy}$ radio sources are identified to $R \sim 27$ in the Subaru/ $X M M$-Newton Deep Field and show that faint and bright radio sources have similar optical colors. This suggests that many of the faint radio sources are passively evolving ellipticals. Using a highly spectroscopically complete (70\%) sample of faint radio sources in the HDF-N, Barger et al. (2007) confirmed that the optical hosts do not vary much with redshift or radio flux density. The optical host properties, coupled with X-ray information, provide evidence for a significant proportion of low-luminosity or obscured AGNs in the faint radio source population (Simpson et al. 2006; Barger et al. 2007). Recent work using radio-based properties, such as morphology, the near-IRto-radio flux density ratio, the mid-IR-to-radio flux density ratio, and radio luminosity, has found that AGNs still contribute a third of the total $1.4 \mathrm{GHz}$ source counts at the faintest flux densities ( $50 \mu$ Jy) (Seymour et al. 2004).

The Hubble Deep Field-South (HDF-S) is an ideal field for studying radio sources because of the wealth of publicly available data. This field has been studied in wavelengths from the radio to UV optical, and spectroscopic and photometric redshifts are available for thousands of galaxies in the HDF$\mathrm{S}$ and surrounding regions (Teplitz et al. 2001; Rudnick et al. 2001; Labbé et al. 2003).

Radio observations of the HDF-S were made between 1998 and 2001 with the Australia Telescope Compact Array (ATCA) using all four available frequency bands. Between 100 and $300 \mathrm{~h}$ of observing at each band yielded images at 1.4, 2.5, 5.2, and $8.7 \mathrm{GHz}$ with maximum sensitivities of $\sim 10 \mu \mathrm{Jy}$ root mean square (rms). A detailed description of the observations, data 
reduction, and initial results was given by Norris et al. (2005) (hereafter Paper I). The full $1.4 \mathrm{GHz}$ catalog and radio source counts were presented in Huynh et al. 2005 (hereafter Paper II). The 2.5, 5.2, and $8.7 \mathrm{GHz}$ catalogs were presented in Huynh et al. (2007a) (hereafter Paper III).

In this paper, we present the optical properties of the Australia Telescope Hubble Deep Field-South (ATHDF-S) radio sources. This paper is organized as follows. In Section 2, we summarize the existing radio and optical images and present the optical counterparts to the ATHDF-S radio sources. Spectroscopic observations by our team and others are presented in Section 3. In Section 4, we present the photometric redshifts derived from the optical imaging. We discuss the photometric properties of the faint radio population in Section 5 .

We assume a Hubble constant of $71 \mathrm{~km} \mathrm{~s}^{-1} \mathrm{Mpc}^{-1}, \Omega_{\mathrm{M}}=$ 0.27 , and $\Omega_{\Lambda}=0.73$ throughout this paper.

\section{IMAGING DATA}

\subsection{Radio}

The radio observations and data reduction are detailed in Papers I-III, but here we provide a brief summary. The observations consist of single pointings centered on right ascension (R.A.) = 2233 25.96, declination (decl.) $=-603809.0$ $(\mathrm{J} 2000.0)(1.4$ and $2.5 \mathrm{GHz})$, and R.A. = 2232 56.22, decl. $=$ -60 3302.7 (J2000.0) (5.2 and 8.7 GHz). The 5.2 and 8.7 GHz observations are centered on the Hubble Space Telescope (HST) Wide Field Planetary Camera (WFPC) field, while the 1.4 and $2.5 \mathrm{GHz}$ observations were pointed halfway between the WFPC field and a bright confusing source to allow the bright source to be well cleaned from the 1.4 and $2.5 \mathrm{GHz}$ images.

We used a wide variety of ATCA configurations to maximize the $u-v$ coverage. The correlator was set to continuum mode $(2 \times 128 \mathrm{MHz}$ bandwidth), with each $128 \mathrm{MHz}$ bandwidth divided into $32 \times 4 \mathrm{MHz}$ channels. The primary flux density calibrator is PKS B1934-638, while secondary gain and phase calibrations were taken throughout our observations using both PKS B2205-636 and PKS B2333-528.

The final images have a maximum sensitivity of $11.0,10.4$, 7.8 , and $11.0 \mu \mathrm{Jy}$ rms at $1.4,2.5,5.2$, and $8.7 \mathrm{GHz}$, respectively. The size of the images varies because the primary beam of the ATCA is smaller at high frequencies. We cataloged $1.4 \mathrm{GHz}$ sources to 20 arcmin distance from the image center, while for 8.7 GHz we reach 3.5 armin distance. Sources were cataloged to a local signal-to-noise ratio $(\mathrm{S} / \mathrm{N})$ of $5 \sigma$ at $1.4,5.2$, and $8.7 \mathrm{GHz}$, and $5.5 \sigma$ at $2.5 \mathrm{GHz}$. A consolidated catalog with the radio sources matched across all four bands was presented in Paper III, comprising 473 individual sources.

\subsection{CTIO Wide Field Images}

Palunas et al. (2000) observed the HDF-S region using the Big Throughput Camera (BTC) on the Cerro Tololo Inter-American Observatory (CTIO) 4 m during 1998 September. Images were taken in the Sloan Digital Sky Survey (SDSS) $u$, Johnson $B$ and $V$, and Cousins $R$ and $I$ filters. The BTC has a pixel size of 0.43 arcsec and covers a total area of $34.8 \times 34.8$ arcmin. Individual exposures were dithered during the observations to fill in the gaps between the BTC CCDs. This resulted in a contiguous field of approximately $44 \times 44$ arcmin, centered on the main HDF-S.

Catalogs of individual sources were compiled by Palunas et al. (2000) using Source Extractor (SExtractor) (Bertin \& Arnouts 1996). Palunas et al. (2000) fix their photometric system

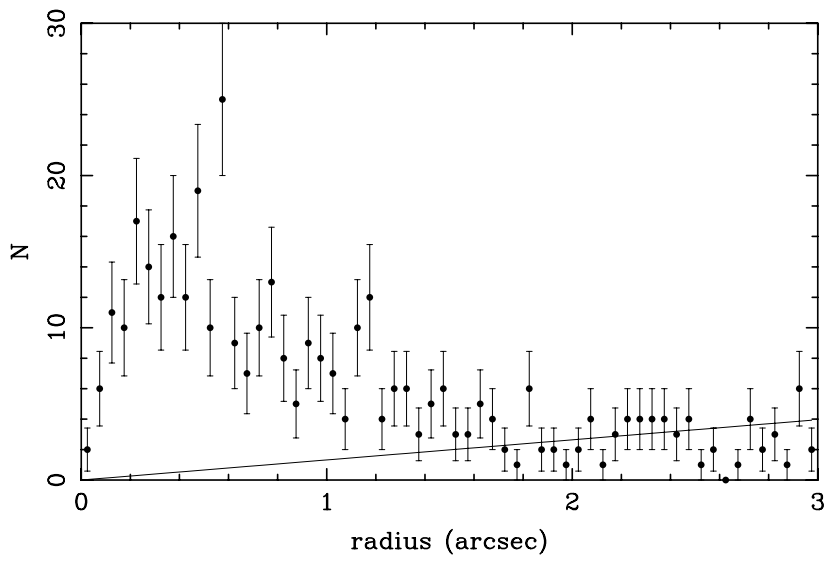

Figure 1. Number of candidate CTIO counterparts of ATHDF-S sources as a function of radio-to-optical offset. The solid line shows the number of chance coincidences expected from the CTIO source density of 70409 objects in a 0.588 square degree region.

to the Johnson $U B V$ and Cousins RI system (Landolt 1992). The sensitivity of the images differ, with the $5 \sigma$ detection limits for point-like objects in each band range quoted as $24<u<$ $25,25.6<B<26.6,25<V<26,25<R<25.8$, and $23.5<$ $I<24.4$. About $50 \%$ of the area of each image has a sensitivity limit at the bright end of the range.

We cross-matched the combined ATHDF-S catalog (Paper III) against the CTIO wide field images. To aid in choosing a matching radius we plot the number of candidate matches and the number of chance coincidences against radio-optical offset in Figure 1. Here the number of chance coincidences is determined from the CTIO source density $(70,409$ cataloged sources over $0.588 \mathrm{deg}^{2}$ ). Figure 1 shows that the number of chance coincidences matches the number of candidates at a radius of 1.8 arcsec. We therefore choose this distance to be our matching radius.

For the worst-case scenario of $\mathrm{S} / \mathrm{N}=5$ and seeing of $2 \operatorname{arcsec}$ FWHM, we estimate the $1 \sigma$ CTIO positional uncertainty to be $\sim 0.4^{\prime \prime}$ in each coordinate. This is similar in scale to the CTIO pixel size. In the ATHDF-S, at $1.4 \mathrm{GHz}$, the average uncertainty is $0.36^{\prime \prime}$ and $0.43^{\prime \prime}$ in R.A. and decl., respectively. We thus expect the offsets between our radio positions and the CTIO positions to have an rms value of approximately $\sqrt{0.4^{2}+0.4^{2}}=0.6^{\prime \prime}$ in each coordinate, neglecting any offsets between the coordinate frames. Figure 2 shows the offsets between the radio and optical positions, which indicates there is a possible R.A. offset in the ATHDF-S and CTIO image coordinates of $\leqslant 0.5$ arcsec. For probable matches closer than 2 arcsec, the mean offset in R.A., $\delta$ R.A., is -0.11 arcsec and the rms is 0.56 arcsec, where $\delta$ R.A. $=$ R.A.ATHDF-S - R.A.CTIO. Similarly, the mean offset in decl. is -0.03 arcsec, with an rms of 0.60 arcsec. We correct for the coordinate shifts between the ATHDF-S and CTIO images by shifting the CTIO positions by these mean offsets in R.A. and decl. before the final matching. The mean offset in R.A. and decl. after shifting the CTIO coordinates is -0.02 and 0.00 arcsec, respectively, with rms values of 0.56 and 0.64 arcsec (Figure 3). This agrees well with the above predicted rms of $0.6^{\prime \prime}$ in each coordinate. Our matching radius of 1.8 arcsec is equivalent to about $2.2 \sigma$, and so should include $\sim 97 \%$ of the true identifications.

The $I$-band CTIO image was examined at all radio positions. In 11 cases the optical counterpart is close to a bright star, so 

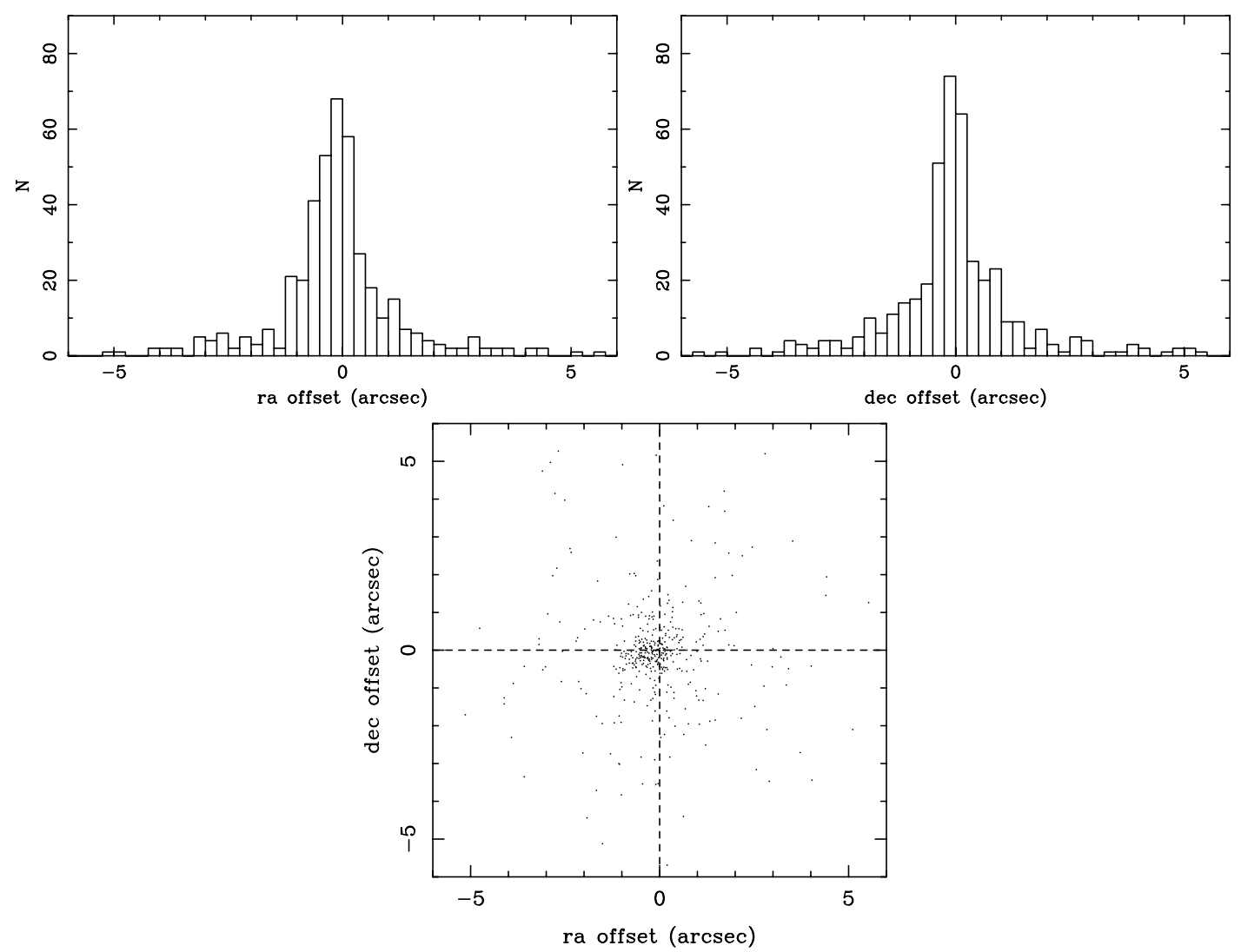

Figure 2. Radio-to-optical position offsets, uncorrected for offset between CTIO and ATHDF-S images. Bottom panel: distribution of the offsets between the radio and optical positions for all ATHDF-S and CTIO sources closer than 6 arcsec. Top panel: distribution of the offset in R.A. and decl. for the CTIO sources closer than 6 arcsec.

the photometry for these sources is not reliable. These instances are flagged in the catalog. For radio sources which comprise a multiple component radio source (see Paper II) the optical counterpart may be positioned between the two or more sources, i.e. at the radio centroid. The two cases where this occurred (multiple sources ATHDF-S_J223232.4-603542 and ATHDFS_J223443.9-602739) are shown in Figure 4.

The number of ATHDF-S sources with a CTIO optical counterpart is 306. Eight of the radio sources lie outside of the CTIO image area, so out of a possible 465 radio sources, $66 \%$ have optical counterparts in the CTIO images. We expect 44 chance coincidences using the CTIO source density of $119,740 \mathrm{deg}^{-2}$ and a total search area of $\left(\pi * 1.8^{\prime 2}\right) \times 473$. To derive another estimate of the number of chance coincidences we shifted the radio source positions north by 0.5 arcmin and re-applied the matching program. From this method we find 55 chance coincidences, which is consistent with our first estimation. We therefore conclude that up to $50 / 315$, or $16 \%$, of our CTIO matches could be spurious.

The results of cross-matching the full ATHDF-S catalog against the CTIO imaging are presented in Table 1. A description of Table 1 is as follows:

Column 1: ATHDF-S source name.

Column 2: CTIO flag. "YES" indicates there is a CTIO counterpart within the 1.8 arcsec matching radius. "NO" means there is no CTIO counterpart within the 1.8 arcsec matching radius. " $\mathrm{S}$ " indicates there is a nearby bright star which may affect the photometry. "OUT" indicates that the source lies outside of the CTIO images.
Column 3: CTIO source ID, corresponding to the source number from the CTIO catalog (P. Palunas et al. 2008, submitted).

Column 4: $U$ magnitude.

Column 5: rms error in $U$ magnitude.

Column 6: $B$ magnitude.

Column 7: rms error in $B$ magnitude.

Column 8: $V$ magnitude.

Column 9: rms error in $V$ magnitude.

Column 10: $R$ magnitude.

Column 11: rms error in $R$ magnitude.

Column 12: I magnitude.

Column 13: rms error in I magnitude.

Column 14: CTIO photometric redshift.

Column 15: CTIO photometric redshift uncertainty.

\subsection{Hubble Space Telescope Images}

Images of the HDF-S were obtained by all three instruments on board the HST in 1998: the Wide Field Planetary Camera 2 (WFPC2), the Near-Infrared Camera and Multi-Object Spectrograph (NICMOS), and the Space Telescope Imaging Spectrograph (STIS). The HST observing strategy is described in Williams et al. (2000).

\subsubsection{Main Fields}

The main deep field WFPC2 observations were centered on R.A. = 2232 56.22, and decl. = -603302.69 (J2000). The WFPC2 observations consist of exposures in four broadband filters: F300W, F450W, F606W, and F814W, which have central 

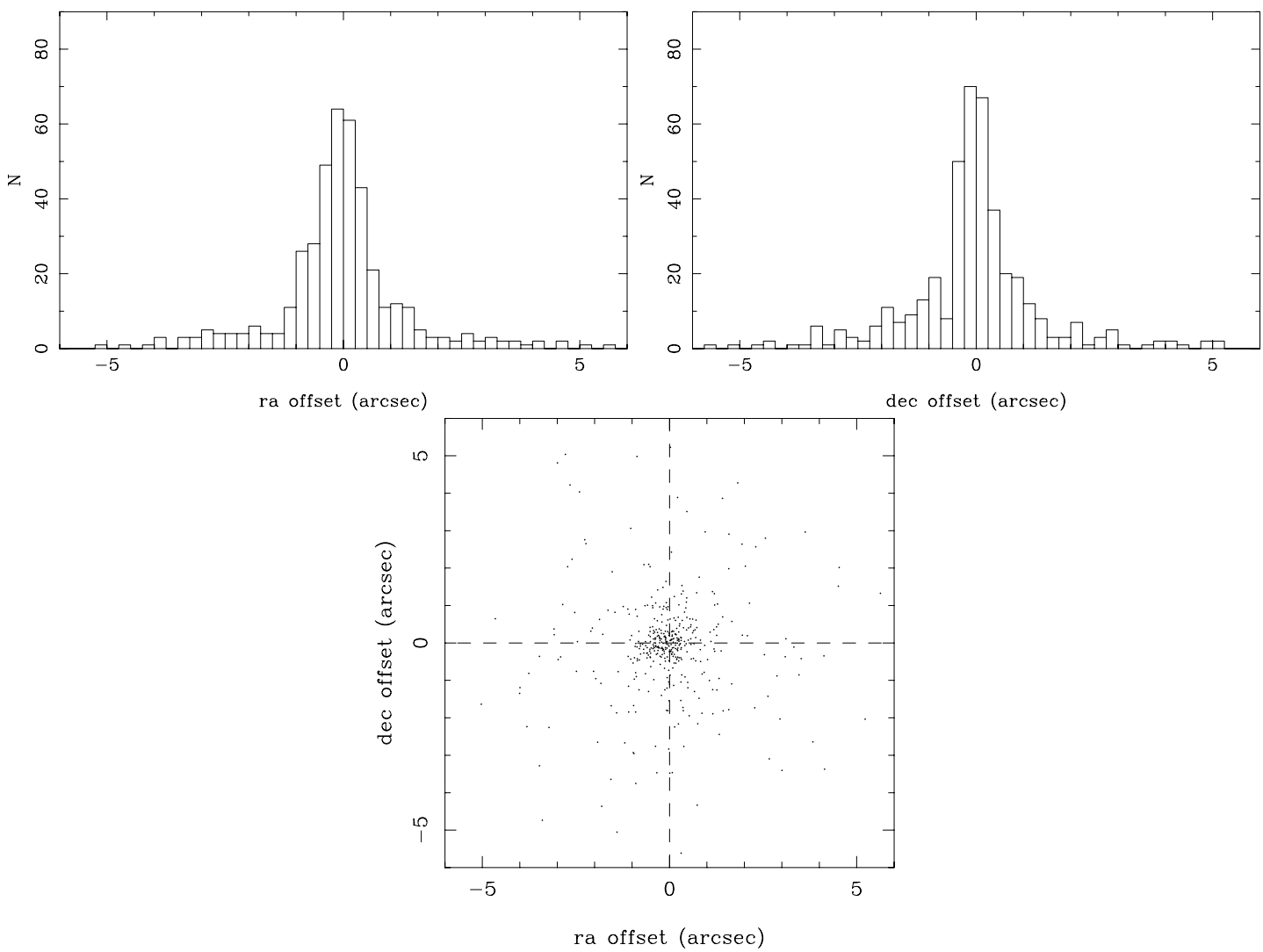

dec offset $(\operatorname{arcsec})$ Figure 3. Radio-to-optical position offsets, with CTIO coordinates shifted to correct for offset between CTIO and ATHDF-S images. Bottom panel: distribution of the CTIO sources closer than 6 arcsec.
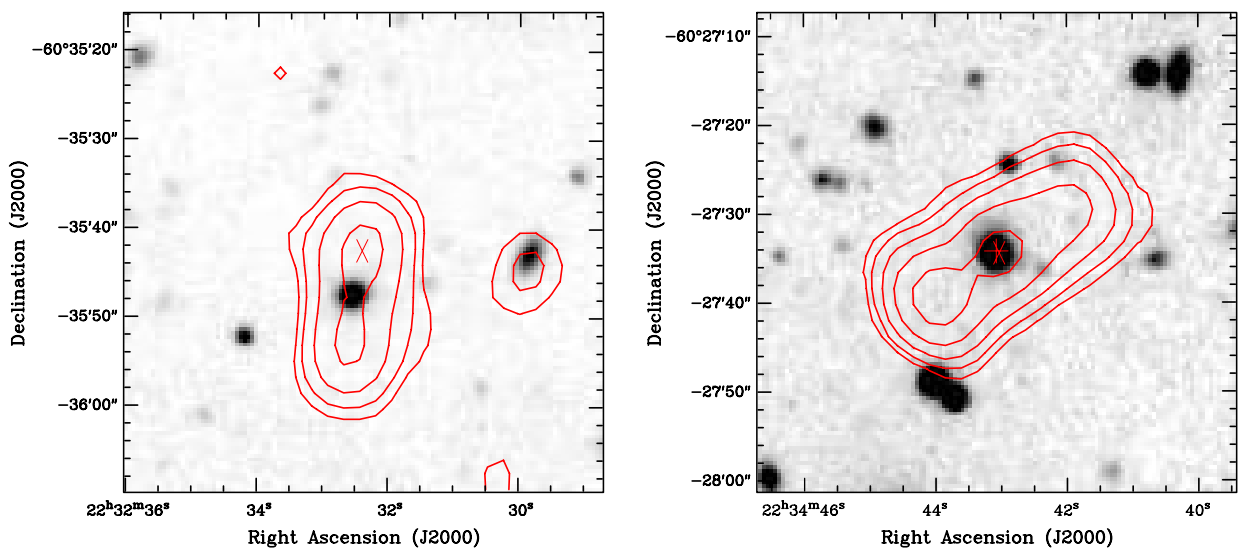

Figure 4. Postage stamp images for radio sources with multiple components. The optical counterparts are clearly positioned between the two or more radio source components. The gray-scale images are CTIO $I$-band images and the contours are $1.4 \mathrm{GHz}$ flux densities, set at 5, 10, 20, 50, 100, and 200 $\sigma$. Left: multiple radio source ATHDF-S_J223232.4-603542. Right: multiple radio source ATHDF-S_J223443.9-602739.

(A color version of this figure is available in the online journal)

wavelengths of approximately 2940, 4520, 5940, and $7920 \AA$, respectively. These filters are the $H S T$ analogs of the well-known Johnson $U, B, V$, and Cousins $I$ filters. The $10 \sigma$ limiting $A B$ magnitude of the WFPC2 observations are 26.8, 27.7, 28.3, and 27.7 for the F300W, F450W, F606W, and F814W filters, respectively (Casertano et al. 2000).

The NICMOS instrument was used to obtain deep near infrared (IR) images. Imaging was performed in three NICMOS filters (Williams et al. 2000): F110W, F160W and F222W. These filters correspond roughly to $J(\mathrm{~F} 110 \mathrm{~W}), H(\mathrm{~F} 160 \mathrm{~W})$, and $K$
$(\mathrm{F} 222 \mathrm{~W})$. The NICMOS field is centered at R.A. $=223251.75$, and decl. $=-603848.20$ (J2000). The sensitivity, while not uniform over the whole image, reaches a limiting F160W $(\mathrm{H})$ $A B$ magnitude of $\equiv 28.7$ and $\mathrm{F} 222 \mathrm{~W}(\mathrm{~K}) A B$ magnitude of $\equiv 24.8$ (Yahata et al. 2000).

The STIS observations were centered on the STIS QSO (Sealey et al. 1998) at R.A. = 2233 37.5883, and decl. = -60 3329.128 (J2000). The imaging was performed mainly with the 50CCD detector in a filterless mode which provides a wide (2500 ̊ FWHM) bandpass centered at approximately 
Table 1

CTIO Properties of the Cataloged ATHDF-S Sources

\begin{tabular}{|c|c|c|c|c|c|c|c|c|c|c|c|c|c|c|}
\hline ATHDF-S name & $\begin{array}{c}\text { CTIO } \\
\text { flag }\end{array}$ & CTIO ID & $U$ mag & $\begin{array}{l}U \text { rms } \\
\text { error }\end{array}$ & $B$ mag & $\begin{array}{l}B \mathrm{rms} \\
\text { error }\end{array}$ & $V$ mag & $\begin{array}{l}V \mathrm{rms} \\
\text { error }\end{array}$ & $R$ mag & $\begin{array}{c}R \mathrm{rms} \\
\text { error }\end{array}$ & $I \mathrm{mag}$ & $\begin{array}{l}I \mathrm{rms} \\
\text { error }\end{array}$ & CTIO phot $z$ & $\begin{array}{c}\text { CTIO phot } z \\
\text { uncertainty }\end{array}$ \\
\hline ATHDFS_J223314.6-605543 & YES & 2689 & 18.11 & 01 & 5 & 00 & 17.42 & 0.00 & 16.98 & 0.00 & 16 & 0.01 & 0 . & 0.05 \\
\hline ATHDFS_J223316.9-605533 & YES & 3290 & 17.13 & 0.01 & 16.88 & 0.00 & 15.96 & 0.00 & 15.37 & 0.00 & 14.72 & 0.00 & 0.17 & 0.11 \\
\hline ATHDFS_J223353.9-605452 & YES & 1648 & 22.99 & 0.16 & 24.05 & 0.18 & 23.00 & 0.15 & 22.41 & 0.12 & 21.23 & 0.19 & 0.98 & 0.25 \\
\hline ATHDFS_J223334.6-605455 & NO & $\ldots$ & $\ldots$ & $\ldots$ & $\ldots$ & . & $\ldots$ & $\ldots$ & $\ldots$ & $\ldots$ & $\ldots$ & $\ldots$ & $\ldots$ & $\ldots$ \\
\hline ATHDFS_J223214.8-605430 & YES & 2073 & 23.10 & 0.16 & 23.74 & 0.12 & 22.67 & 0.08 & 22.28 & 0.09 & 21.58 & 0.22 & 0.27 & 0.18 \\
\hline ATHDFS_J223448.4-605417 & YES & 2430 & 23.48 & 0.50 & 24.58 & 0.61 & 24.31 & 0.82 & 22.89 & 0.42 & 22.11 & 0.94 & 0.71 & 0.35 \\
\hline ATHDFS_J223317.5-605416A & NO & $\ldots$ & $\ldots$ & $\ldots$ & $\ldots$ & $\ldots$ & $\ldots$ & $\ldots$ & $\ldots$ & .. & $\ldots$ & $\ldots$ & $\ldots$ & $\ldots$ \\
\hline ATHDFS_J223317.5-605416B & NO & $\ldots$ & $\ldots$ & $\ldots$ & $\ldots$ & $\ldots$ & $\ldots$ & $\ldots$ & $\ldots$ & $\ldots$ & $\ldots$ & $\ldots$ & $\ldots$ & $\ldots$ \\
\hline ATHDFS_J223156.0-605417 & NO & $\ldots$ & $\ldots$ & $\ldots$ & $\ldots$ & $\ldots$ & $\ldots$ & $\ldots$ & $\ldots$ & $\ldots$ & $\ldots$ & $\ldots$ & $\ldots$ & $\ldots$ \\
\hline ATHDFS_J223204.8-605414 & YES & 2735 & 20.14 & 0.02 & 20.52 & 0.01 & 19.50 & 0.01 & 18.94 & 0.01 & 18.28 & 0.02 & 0.21 & 0.15 \\
\hline
\end{tabular}

(This table is available in its entirety in a machine-readable form in the online journal. A portion is shown here for guidance regarding its form and content)

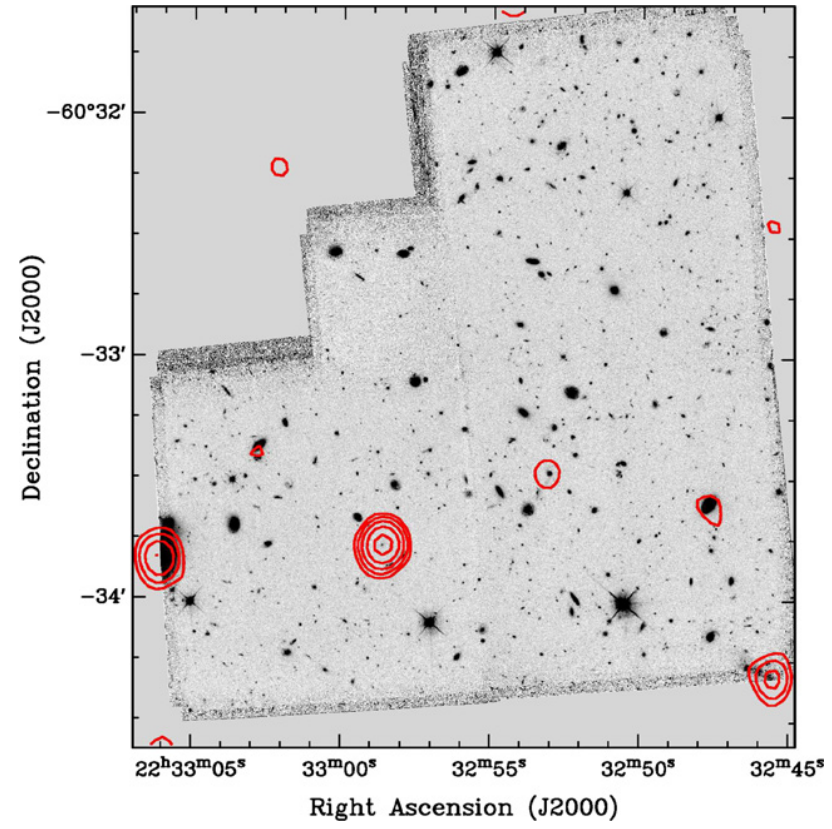

Figure 5. HDF-S main WFPC2 field-F814W image. The contours are $1.4 \mathrm{GHz}$ flux densities, set at 5, 10, 20, 50,100, and $200 \sigma$.

(A color version of this figure is available in the online journal)

5800 Å. Rough color information was obtained by observations with the F28X50LP filter which is a long-pass filter beginning at approximately $5500 \AA$. These images cover a square region $0.83 \mathrm{arcmin}^{2}$ in total with a resolution of 0.05 per pixel. The filterless 50CCD images roughly correspond to $V+I$ and reach a depth of 29.4 $A B$ magnitudes (Gardner et al. 2000).

Figures 5 to 7 show the full HST deep images overlaid with ATHDF-S $1.4 \mathrm{GHz}$ contours. Four of the ATHDF-S radio sources lie within the WFPC2 field, one is in the NICMOS field, and two lie in the STIS field (Tables 2-4). All four sources within WFPC have a counterpart within 1.5 arcsec, and the others have counterparts within 0.5 arcsec. Using the WFPC 2 source density of 2657 objects over $5.07 \mathrm{arcmin}^{2}$, we expect only one of these alignments, at most, is due to chance. The gray-scale postage stamp images of each source are presented in Figure 8 .

\subsubsection{Flanking Fields}

In addition to the main deep field exposures, a series of shallower observations were carried out over 27 orbits of the

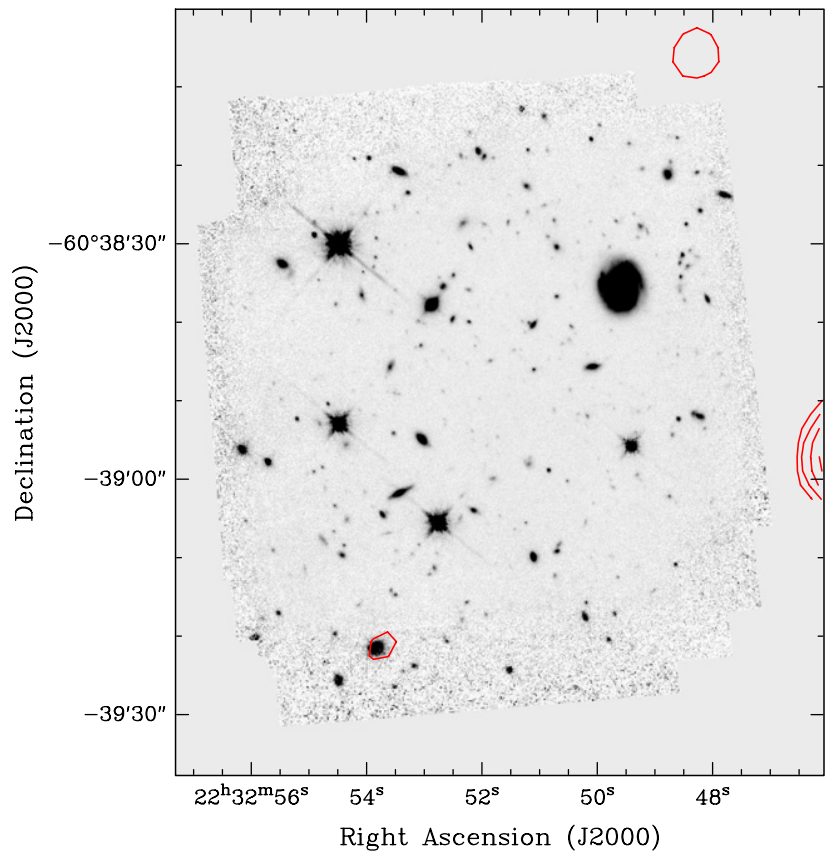

Figure 6. HDF-S main NICMOS field-F160W image. The contours are 1.4 $\mathrm{GHz}$ flux densities, set at 5, 10, 20,50,100, and $200 \sigma$.

(A color version of this figure is available in the online journal)

$H S T$. Nine two-orbit WFPC2 pointings were used to observe a contiguous area of sky between the deep STIS, WFPC2, and NICMOS fields, overlapping both the STIS and NICMOS deep fields (Williams et al. 2000). Each of the WFPC2 flanking field exposures has simultaneous STIS and NICMOS exposures. The WFPC2 observations were performed in the $I$-band (F814W), while the STIS images were with the filterless 50CCD configuration and NICMOS images were in the $H$-band (F160W). While sensitivity varies slightly between the images, the average $5 \sigma$ limiting $A B$ magnitude for the flanking field images is $I$ $(\mathrm{F} 814 \mathrm{~W})=26.02, H(\mathrm{~F} 160 \mathrm{~W})=26.32$, and $V+I(50 \mathrm{CCD})=$ 28.24 for the WFPC2, NICMOS, and STIS images, respectively (Lucas et al. 2003).

A deep nine-orbit STIS filterless 50CCD image of the main NICMOS field was also obtained as part of the flanking fields program. This was in order to obtain rough color information on NICMOS objects. The STIS image reaches a $5 \sigma A B$ magnitude of $V+I=29.09$ (Lucas et al. 2003). Simultaneous exposures by WFPC 2 achieve $V(\mathrm{~F} 606 \mathrm{~W})=27.79$ and $I(\mathrm{~F} 814 \mathrm{~W})=27.15$, 
Table 2

Summary of WFPC2 Counterparts

\begin{tabular}{lcccccccrc}
\hline \hline ATHDF-S name & $\begin{array}{c}\text { F300 } \\
\text { magnitude }\end{array}$ & $\begin{array}{c}\text { F300 } \\
\text { rms error }\end{array}$ & $\begin{array}{c}\text { F450 } \\
\text { magnitude }\end{array}$ & $\begin{array}{c}\text { F450 } \\
\text { rms error }\end{array}$ & $\begin{array}{c}\text { F606 } \\
\text { magnitude }\end{array}$ & $\begin{array}{c}\text { F606 } \\
\text { rms error }\end{array}$ & $\begin{array}{c}\text { F814 } \\
\text { magnitude }\end{array}$ & $\begin{array}{c}\text { F814 } \\
\text { rms error }\end{array}$ & $\begin{array}{c}\text { Morphological } \\
\text { information }\end{array}$ \\
\hline ATHDF-S_J223258.6-603346 & 27.53 & 0.68 & 28.48 & 0.77 & 27.05 & 0.13 & 25.12 & 0.04 & No info \\
ATHDF-S_J223247.6-603337 & 22.70 & 0.02 & 21.69 & 0.00 & 20.55 & 0.00 & 19.55 & 0.00 & Late-type spiral \\
ATHDF-S_J223253.1-603329 & 25.00 & 0.07 & 24.31 & 0.02 & 23.69 & 0.01 & 22.56 & 0.00 & No info \\
ATHDF-S_J223302.8-603323 & 25.45 & 0.19 & 23.60 & 0.02 & 23.36 & 0.01 & 22.79 & 0.01 & Barred spiral \\
\hline
\end{tabular}

Table 3

Summary of NICMOS Counterparts

\begin{tabular}{lccccccc}
\hline \hline ATHDF-S name & $\begin{array}{c}\text { F110 } \\
\text { magnitude }\end{array}$ & $\begin{array}{c}\text { F110 } \\
\text { rms error }\end{array}$ & $\begin{array}{c}\text { F160 } \\
\text { magnitude }\end{array}$ & $\begin{array}{c}\text { F160 } \\
\text { rms error }\end{array}$ & $\begin{array}{c}\text { F222 } \\
\text { magnitude }\end{array}$ & $\begin{array}{c}\text { F222 } \\
\text { rms error }\end{array}$ & $\begin{array}{c}\text { Morphological } \\
\text { information }\end{array}$ \\
\hline ATHDF-S_J223253.7-603921 & 21.20 & 0.05 & 19.97 & 0.02 & 19.38 & 0.07 & No info \\
\hline
\end{tabular}

Table 4

Summary of STIS Counterparts

\begin{tabular}{lccccccccc}
\hline \hline ATHDF-S name & $\begin{array}{c}\text { 50ccd } \\
\text { magnitude }\end{array}$ & $\begin{array}{c}\text { 50ccd } \\
\text { rms error }\end{array}$ & $\begin{array}{c}\text { lp } \\
\text { magnitude }\end{array}$ & $\begin{array}{c}\text { lp } \\
\text { rms error }\end{array}$ & $\begin{array}{c}\text { NUV } \\
\text { magnitude }\end{array}$ & $\begin{array}{c}\text { NUV } \\
\text { rms error }\end{array}$ & $\begin{array}{c}\text { FUV } \\
\text { magnitude }\end{array}$ & $\begin{array}{c}\text { FUV } \\
\text { rms error }\end{array}$ & $\begin{array}{c}\text { Morphological } \\
\text { information }\end{array}$ \\
\hline ATHDF-S_J223337.5-603329 & 17.83 & 0.0 & 17.48 & 0.00 & $\ldots$ & $\ldots$ & 29.13 & 0.00 & $\begin{array}{c}\text { Bright point source } \\
\text { (STIS QSO) }\end{array}$ \\
ATHDF-S_J223339.4-603306 & 24.76 & 0.01 & 24.46 & 0.04 & 41.00 & 0.00 & 37.33 & 0.00 & $\begin{array}{c}\text { Irregular and disturbed, } \\
\text { Possible merging system }\end{array}$
\end{tabular}

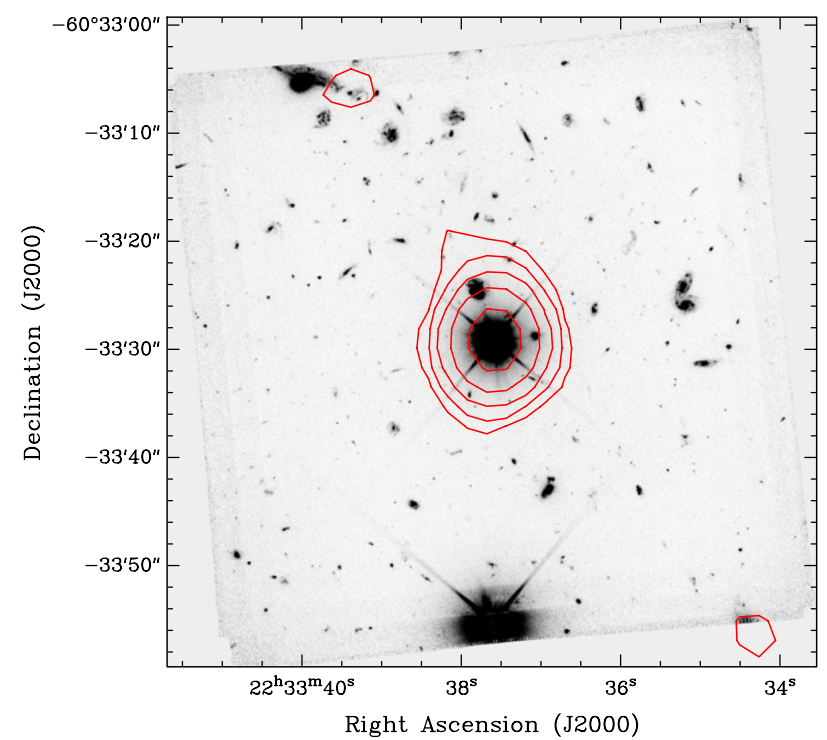

Figure 7. HDF-S main STIS field-50CCD image. The contours are $1.4 \mathrm{GHz}$ flux densities, set at 5, 10, 20, 50, 100, and 200 $\sigma$.

(A color version of this figure is available in the online journal)

and the simultaneous NICMOS imaging depth is $J(\mathrm{~F} 110 \mathrm{~W})=$ 26.94 and $H(\mathrm{~F} 160 \mathrm{~W})=26.66$ (Lucas et al. 2003).

We cross-matched the positions of our ATHDF-S radio sources with the full flanking field catalogs (Lucas et al. 2003). Flanking field objects were considered probable counterparts if the positional offset was less than three times the radio positional uncertainty. The largest offset accepted as a match is for ATHDF-S_J223307.1-603448, where the WFPC2 counterpart is radially offset from the radio position by $1.96^{\prime \prime}$. In Table 5 we summarize the properties of the counterparts found in the STIS, NICMOS, and WFPC2 flanking fields, respectively. The grayscale $H S T$ postage stamp images with overlaid radio contours for all flanking field counterparts are presented in Figures 9-11.

\subsubsection{Analysis of HST Counterparts and Optically Faint Microjansky Sources}

In Section 2.2, we found that $66 \%$ of our ATHDF-S sources are detected by CTIO imaging. In the main WFPC2 field, the HST imaging achieves sensitivities of 4.2 mag deeper than CTIO in the F814W (pseudo- $I$ ) band. In the flanking fields, the $H S T$ goes 2.5 mag deeper in $I$. Thus, we expect the HST imaging to detect more of our radio sources. The HST imaging can also be used to confirm that the CTIO imaging is sensitive to $I=23.5$.

Table 6 lists the CTIO and HST I magnitudes of the ATHDF$\mathrm{S}$ sources which lie in the WFPC2 deep and flanking fields. There are 35 radio sources within this region, and of these, 22 have CTIO counterparts. Five of the 13 sources without CTIO counterparts (i.e. $I$ mag $>23.5$ ) are identified in the WFPC2 imaging $(\mathrm{F} 814 \mathrm{~W}<26.0)$. If the sample within the WFPC2 deep and flanking fields is representative of the full ATHDF-S radio sample, then $67 \%$ of our radio sources are detected to $I$ mag $=$ 23.5 (CTIO limit) and $79 \%$ to $I$ mag $~ 26.0$ (WFPC2 limit). This is consistent with the HDF-N and small science aperture (SSA) 13 fields, where a sub-mJy radio source identification rate of $80 \%$ was achieved for a limit of $I$ mag $=25.0$ (Richards et al. 1999).

This implies that there is a sizable proportion of sub-mJy radio sources with very faint optical counterparts $\left(I_{\mathrm{AB}}>26\right)$. We use the I mag limit to calculate the lower limit to the radio-to-optical ratio (see Section 5.2) of these sources. Table 7 lists the $1.4 \mathrm{GHz}$ flux density and radio-to-optical ratio $\left(R_{I}\right)$ limit for these eight sources. We find that all of these optically unidentified sources have relatively high radio-to-optical ratios $\left(R_{I}>2.5\right)$. Four sources have $R_{I}>3$, which meets the criteria for radio-loud AGNs discussed in Section 5.2. Redshifts are required before we can firmly rule out star-formation processes as the source of the radio emission. However, we do note that these sources may be similar to the optically faint microjansky sources identified in radio observations of the HDF-N and SSA 13 fields (Richards et al. 1999). 


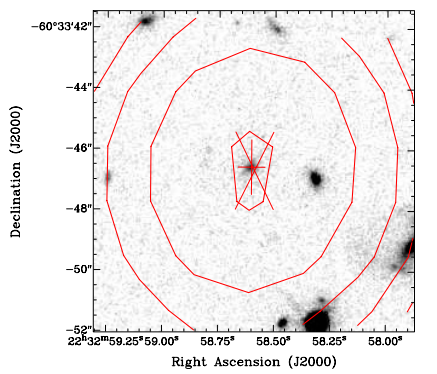

A T H D F S_J $223258.5-603346$

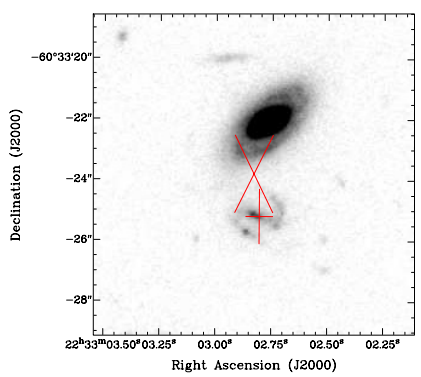

A T H D F S _J $223302.8-603323$

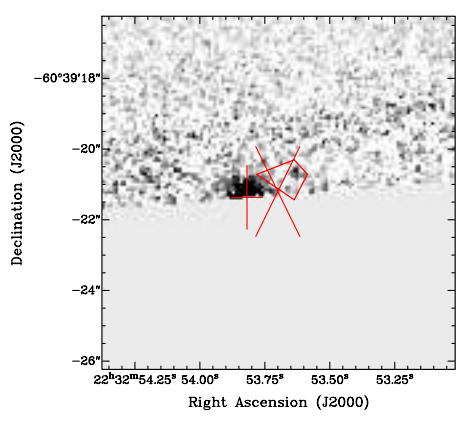

A T H D F S _J $223253.7-603921$

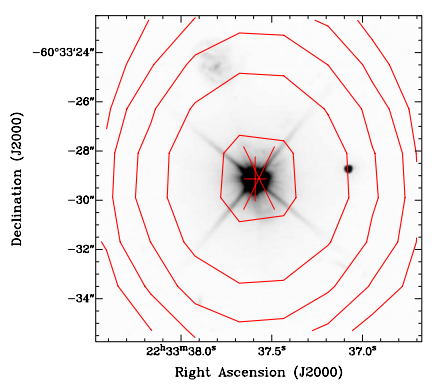

A T H D F S_J $223337.5-603329$
WFPC2 814W
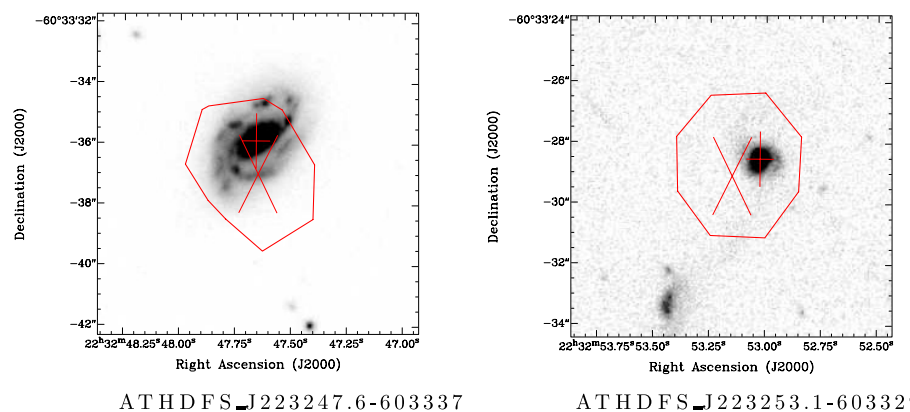

A T H D F S J $223253.1-603329$

NICMOS F160W

STIS 50CCD

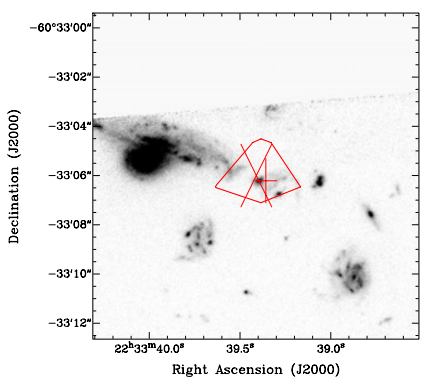

A T H D F S _J $223339.4-603306$

Figure 8. Gray-scale postage stamp images of the HST deep field counterparts to ATHDF-S radio sources. The crosses mark the radio position and the plus signs

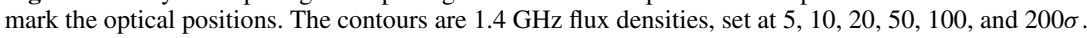

(A color version of this figure is available in the online journal)

Because of their faintness, not much is known about optically faint microjansky sources, but Richards et al. (1999) propose several possibilities for the nature of these radio sources: (1) luminous dust-enshrouded starbursts at high redshifts of $z=$ 1-3, (2) extremely high redshift AGNs $(z>6)$, or (3) luminous obscured AGNs at $z \gtrsim 2$. Submillimeter observations have shown that a significant fraction of these sources (at least $30 \%$ ) are dusty starbursts at $1<z<3$ (Barger et al. 2000;
Chapman et al. 2001, 2003). A large fraction ( 50\%) of the population also has detectable X-ray emission (Alexander et al. 2001), and the majority of these X-ray-detected sources have obscured AGN activity. A stacking analysis performed on the optically faint microjansky sources not detected by current $\mathrm{X}$-ray observations found that their average X-ray properties are consistent with an object like Arp220 lying at $z \sim 1.5$ (Alexander et al. 2001). Recent high-resolution radio images 
Table 5

Summary of HST Flanking Field Counterparts

\begin{tabular}{|c|c|c|c|c|c|c|}
\hline \multicolumn{7}{|c|}{ WFPC2 flanking observations } \\
\hline ATHDF-S name & $\begin{array}{l}\text { Flanking field } \\
\text { ID }\end{array}$ & $\begin{array}{c}\text { F606 } \\
\text { magnitude }\end{array}$ & $\begin{array}{c}\text { F606 } \\
\text { rms error }\end{array}$ & $\begin{array}{c}\text { F814 } \\
\text { magnitude }\end{array}$ & $\begin{array}{c}\text { F814 } \\
\text { rms error }\end{array}$ & $\begin{array}{l}\text { Morphological } \\
\text { information }\end{array}$ \\
\hline ATHDF-S_J223207.9-603928 & WdpVI & 26.46 & 0.21 & 25.61 & 0.15 & No info \\
\hline ATHDF-S_J223253.7-603921 & W9 & $\ldots$ & $\ldots$ & 21.12 & 0.01 & Possible merging system \\
\hline ATHDF-S_J223245.6-603857 & W9 & $\ldots$ & $\ldots$ & 23.91 & 0.04 & No info \\
\hline ATHDF-S_J223248.2-603805 & W9 & $\ldots$ & $\ldots$ & 20.90 & 0.01 & Edge-on disk galaxy \\
\hline ATHDF-S_J223254.5-603748 & W9 & $\ldots$ & $\ldots$ & 18.44 & 0.00 & Barred spiral \\
\hline ATHDF-S_J223202.5-603714 & WdpVI & 25.86 & 0.11 & 24.17 & 0.04 & Possible merging system \\
\hline ATHDF-S_J223253.0-603539 & W6 & $\ldots$ & $\ldots$ & 23.45 & 0.04 & No info \\
\hline ATHDF-S_J223245.3-603537 & W6 & $\ldots$ & $\ldots$ & 24.04 & 0.06 & No info \\
\hline ATHDF-S_J223338.8-603523 & W8 & $\ldots$ & $\ldots$ & 18.99 & 0.00 & Early-type spiral \\
\hline ATHDF-S_J223344.9-603515 & W8 & $\ldots$ & $\ldots$ & 19.51 & 0.00 & Spiral galaxy in merging system \\
\hline ATHDF-S_J223350.5-603503 & W8 & $\ldots$ & $\ldots$ & 26.24 & 0.19 & No info \\
\hline ATHDF-S_J223307.1-603448 & W3 & $\ldots$ & $\ldots$ & 24.83 & 0.08 & No info \\
\hline ATHDF-S_J223243.3-603443 & W2 & $\ldots$ & $\ldots$ & 19.32 & 0.00 & Merging system \\
\hline ATHDF-S_J223245.5-603419 & W2 & $\ldots$ & $\ldots$ & 21.10 & 0.01 & Possible merger \\
\hline ATHDF-S_J223311.9-603417 & W3 & $\ldots$ & $\ldots$ & 23.93 & 0.05 & No info \\
\hline ATHDF-S_J223306.0-603350 & W3 & $\ldots$ & $\ldots$ & 17.25 & 0.00 & Face-on spiral \\
\hline ATHDF-S_J223243.4-603352 & W2 & $\ldots$ & $\ldots$ & 19.87 & 0.00 & Point-like, quasar \\
\hline ATHDF-S_J223247.6-603337 & W2 & $\ldots$ & $\ldots$ & 19.64 & 0.01 & Spiral \\
\hline ATHDF-S_J223337.5-603329 & W8 & $\ldots$ & $\ldots$ & 17.70 & 0.00 & Bright point source (STIS QSO) \\
\hline ATHDF-S_J223339.4-603306 & W8 & $\ldots$ & $\ldots$ & 24.81 & 0.10 & No info \\
\hline ATHDF-S_J223308.6-603251 & W1 & $\ldots$ & $\ldots$ & 21.03 & 0.01 & Spheroidal \\
\hline ATHDF-S_J223317.7-603235 & W4 & $\ldots$ & $\ldots$ & 20.83 & 0.01 & Spheroidal \\
\hline ATHDF-S_J223331.6-603222 & W7 & $\ldots$ & $\ldots$ & 20.38 & 0.01 & Merging system, triple nuclei \\
\hline ATHDF-S_J223303.1-603132 & W1 & & $\cdots$ & 24.05 & 0.05 & No info \\
\hline \multicolumn{7}{|c|}{ NICMOS flanking observations } \\
\hline ATHDF-S name & $\begin{array}{c}\text { Flanking field } \\
\text { ID }\end{array}$ & $\begin{array}{c}\text { F111 } \\
\text { magnitude }\end{array}$ & $\begin{array}{c}\text { F111 } \\
\text { rms error }\end{array}$ & $\begin{array}{c}\text { F160 } \\
\text { magnitude }\end{array}$ & $\begin{array}{c}\text { F160 } \\
\text { rms error }\end{array}$ & $\begin{array}{l}\text { Morphological } \\
\text { information }\end{array}$ \\
\hline ATHDF-S_J223250.6-604336 & N9 & $\ldots$ & $\ldots$ & 22.06 & 0.02 & Possible merging system \\
\hline ATHDF-S_J223337.5-604006 & N8 & $\ldots$ & $\ldots$ & 19.66 & 0.00 & Merging system \\
\hline ATHDF-S_J223334.4-603804 & N7 & $\ldots$ & $\ldots$ & 22.30 & 0.09 & No info (at edge of image) \\
\hline ATHDF-S_J223210.3-604433 & $\mathrm{dpJH}$ & 20.45 & 0.03 & 19.83 & 0.01 & No info (at edge of image) \\
\hline \multicolumn{7}{|c|}{ STIS flanking observations } \\
\hline ATHDF-S name & $\begin{array}{l}\text { Flanking field } \\
\text { ID }\end{array}$ & $\begin{array}{c}50 \mathrm{ccd} \\
\text { magnitude }\end{array}$ & $\begin{array}{l}50 \mathrm{ccd} \\
\text { rms error }\end{array}$ & & & $\begin{array}{l}\text { Morphological } \\
\text { information }\end{array}$ \\
\hline ATHDF-S_J223425.0-603452 & S8 & 23.18 & 0.01 & & & Disturbed, merging system \\
\hline ATHDF-S_J223327.6-603414 & $\mathrm{S} 2$ & 28.17 & 0.18 & & & No info \\
\hline
\end{tabular}

suggest that optically faint microjansky sources are examples of dusty high redshift starbursts, and some of these seem to be composite sources with an embedded AGN (Muxlow et al. 2005).

\section{SPECTROSCOPIC DATA}

\subsection{Spectroscopy in the Literature}

The AAO undertook a public domain redshift survey of the HDF-S using the upgraded low dispersion survey spectrograph (LDSS++). The HDF-S LDSS++ targets were selected from deep Anglo-Australian Telescope (AAT) Prime Focus Imaging to $R<24$ of a $9 \times 3$ arcmin field covering the main WFPC2 and STIS HST fields (Glazebrook et al. 2006). A total of 225 targets were observed simultaneously. The spectra obtained have $8 \AA$ spectral resolution and cover the wavelength range from $5300 \AA$ to $10700 \AA$. Four radio sources have high quality (two or more lines) LDSS++ spectra, and we summarize the LDSS++ counterparts in Table 8 . The spectra were examined and typed using the same criteria and nomenclature as the $2 \mathrm{dF}$ spectroscopy.

Spectroscopy was also obtained at the Very Large Telescope (VLT) using the FORS2 spectrograph in the multi-object spec- troscopy (MOS) mode. A total of 194 galaxies in the main HDF-S and flanking fields were targeted during 2000 and 2002. Reliable redshifts, determined from multiple spectral features or the [O II] $3727 \AA$ doublet, were obtained for 97 targets (Sawicki \& Mallén-Ornelas 2003). Approximately half of all galaxies brighter than $\mathrm{F} 814 \mathrm{~W}(\mathrm{AB})=24$ in the HDF-S proper were observed, and redshifts were obtained for $76 \%$. We cross-matched the ATHDF-S radio sources against the Sawicki \& MallénOrnelas (2003) redshift catalog. A total of five counterparts were found and Table 9 summarizes the results.

\section{2. $2 d F$ Optical Spectroscopy}

The public domain spectroscopic follow-up of galaxies in the HDF-S and the surrounding region has yielded about 400 redshifts (Vanzella et al. 2002, Sawicki \& Mallén-Ornelas 2003, Glazebrook et al. 2006). Only six of our radio sources have spectroscopic redshifts from the literature, however. The main factor limiting the spectroscopic completeness is that these redshift surveys were concentrated on the central WFPC2 field and the surrounding HST flanking fields, whereas our radio sources are from a region 40 arcmin in diameter. Hence, we undertook wide-field spectroscopic observations. 


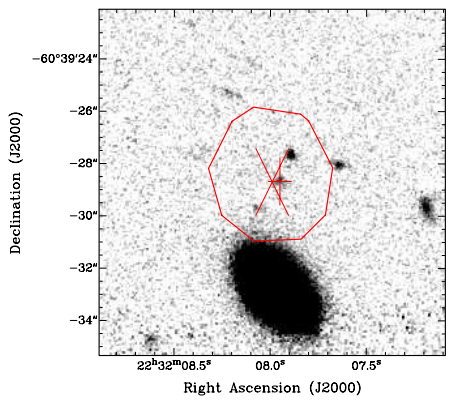

A T H D F S_J 223207.9-603928

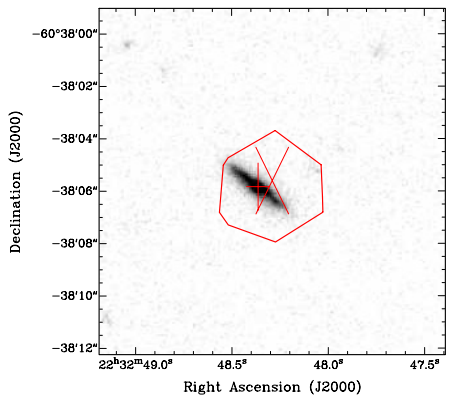

A T H D F S_J $223248.2-603805$

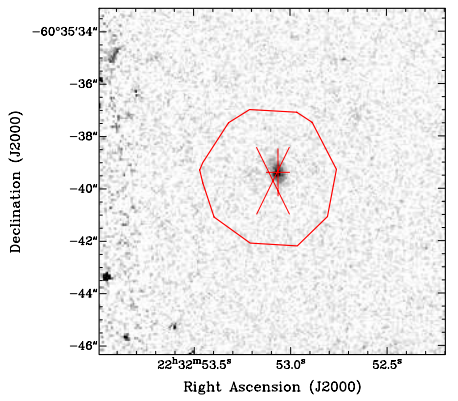

A T H D F S_J 223253.0-603539

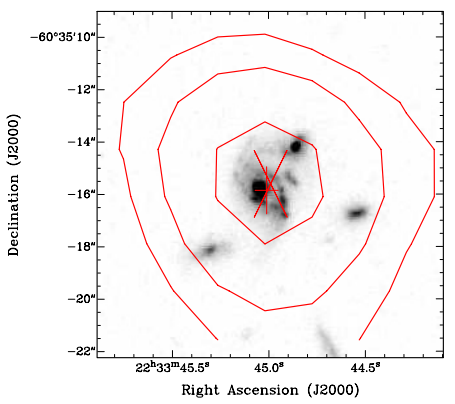

A T H D F S_J $223344.9-603515$

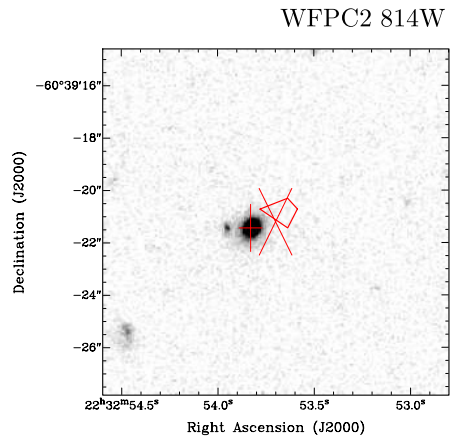

A T H D F S _J $223253.7-603921$

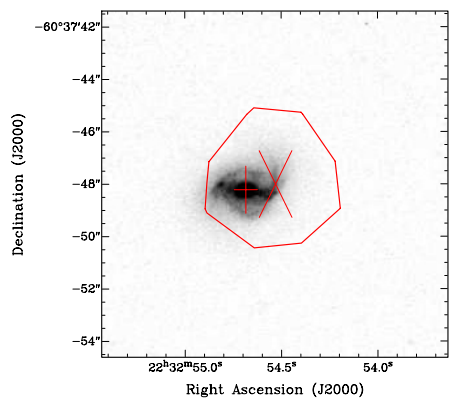

A T H D F S _J $223254.5-603748$

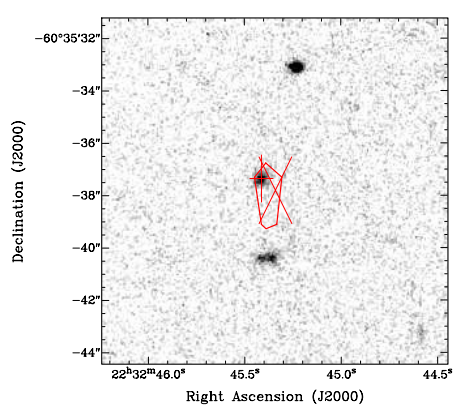

A T H D F S _J $223245.3-603537$

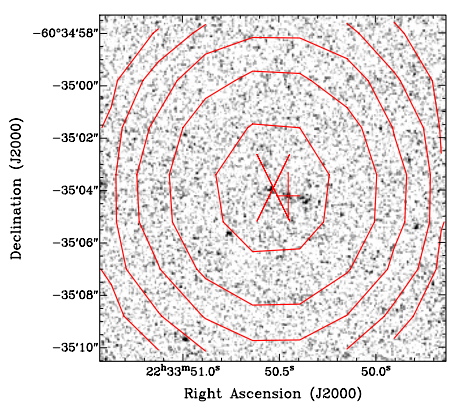

A T H D F S_J $223350.5-603503$

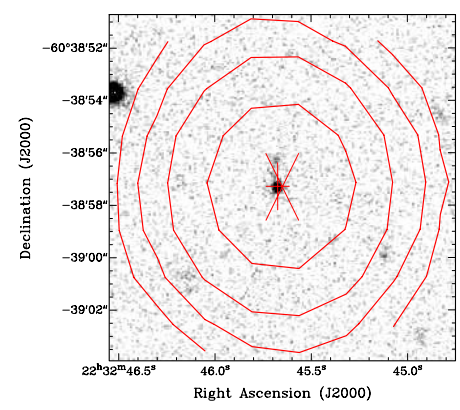

A T H D F S_J $223245,6-603857$

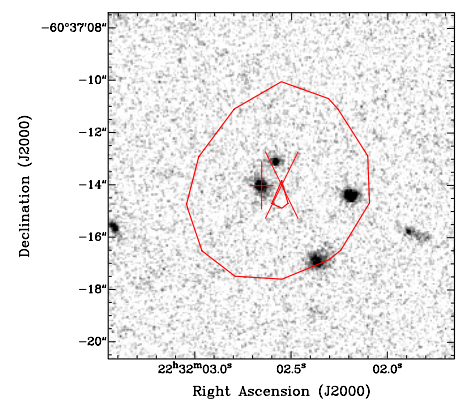

A T H D F S_J $223202.5-603714$

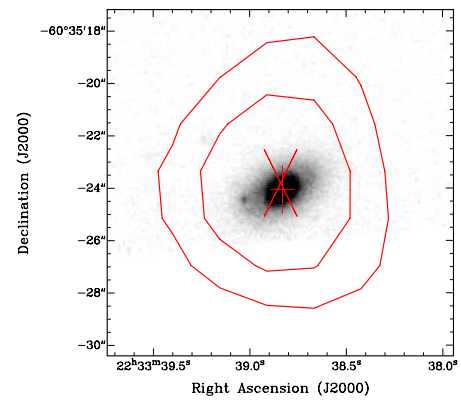

A T H D F S_J $223338.8-603523$

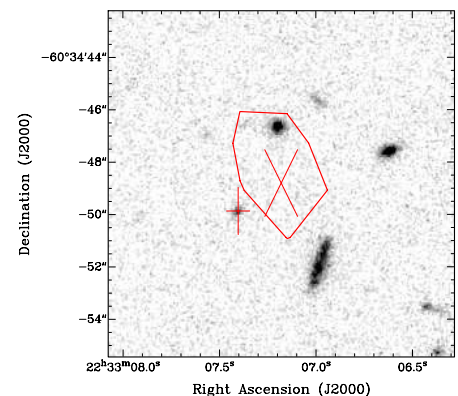

A T H D F S_J $223307.1-603448$

Figure 9. Gray-scale postage stamp images of the HST WFPC2 flanking field counterparts to ATHDF-S radio sources. The crosses mark the radio position and the

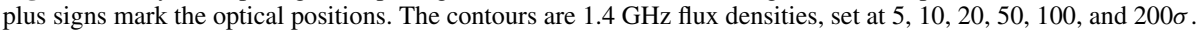

(A color version of this figure is available in the online journal)

We obtained spectra of the ATHDF-S radio sources over two service nights in 2001 July and 2003 October using the multi-fiber $2 \mathrm{dF}$ instrument of the AAT. The $2 \mathrm{dF}$ allows the simultaneous acquisition of up to 400 spectra over a 2 deg diameter region of sky. We employed the 2dFGRS (Colless et al. 2001) observing strategy, which is to use a 300 line $\mathrm{mm}^{-1}$ grating at a central wavelength of $5800 \AA$. This yielded lowresolution (9 $\AA$ ) spectra over the wavelength range 3800 $8000 \AA$. Our data consisted of six consecutive 20 min exposures, giving a total of $2 \mathrm{~h}$ on source. 


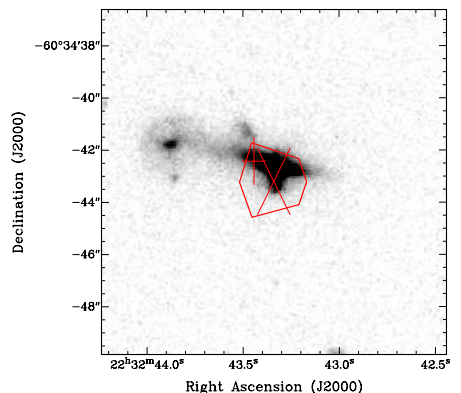

A T H D F S_J $223243.3-603443$

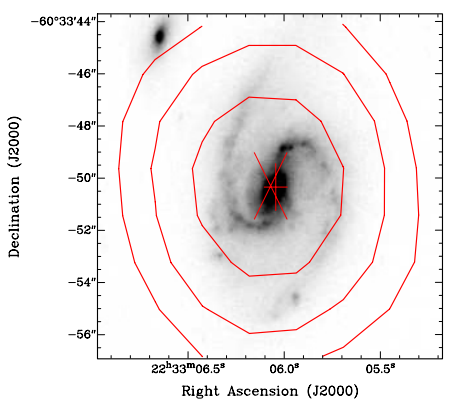

A T H D F S_J $223306.0-603350$

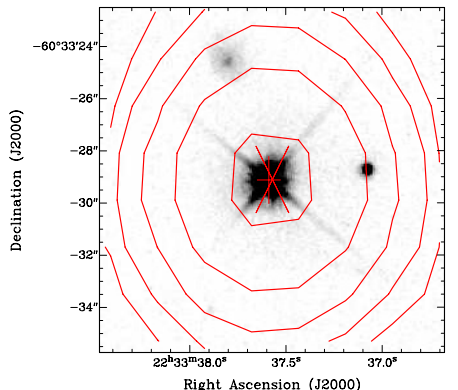

A T H D F S_J $223337.5-603329$

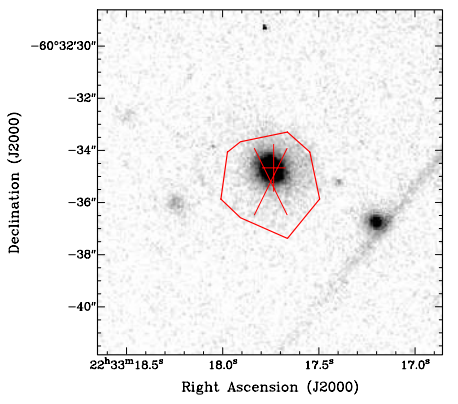

A T H D F S_J $223317.7-603235$

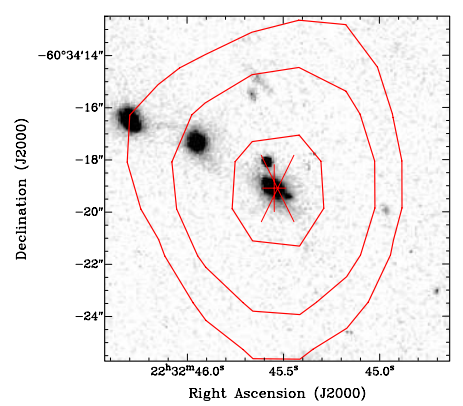

A T H D F S_J $223245.5-603419$

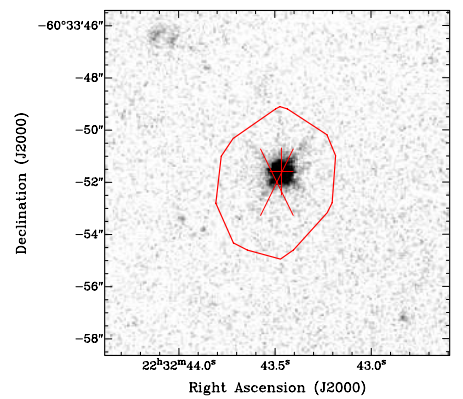

A T H D F S_J $223243.4-603352$

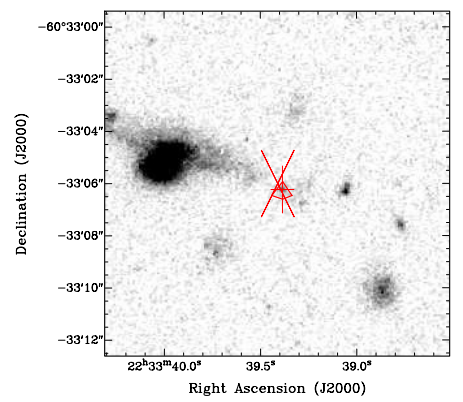

A T H D F S_J 223339.4-603306

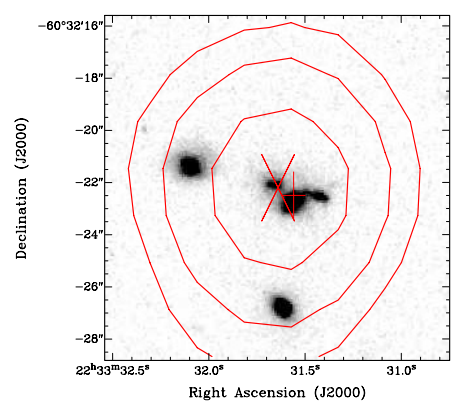

A T H D F S_J $223331.6-603222$

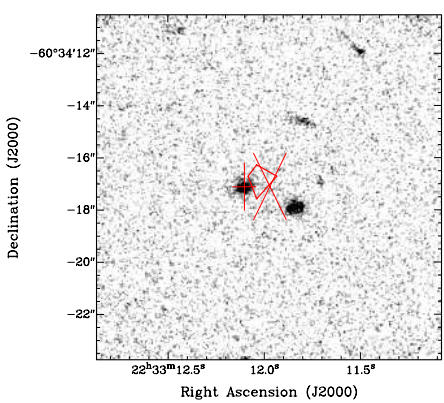

A T H D F S _J $223311.9-603417$

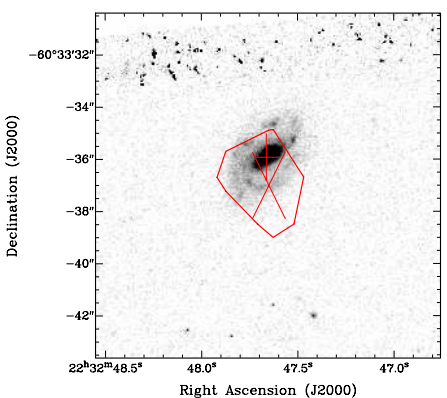

A T H D F __J $223247.6-603337$

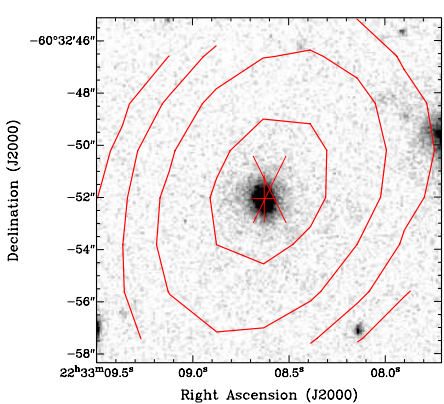

A T H D F S _J $223308.6-603251$

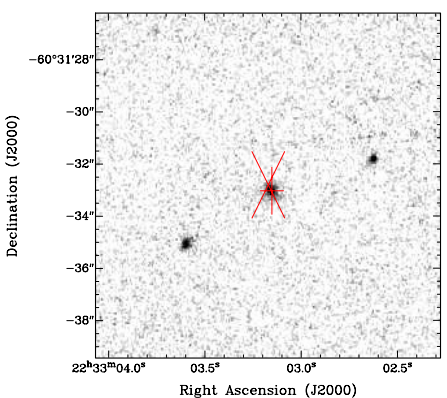

A T H D F S _J $223303.1-603132$

Figure 10. More gray-scale postage stamp images of the HST WFPC2 flanking field counterparts to ATHDF-S radio sources. The crosses mark the radio position and the plus signs mark the optical positions. The contours are $1.4 \mathrm{GHz}$ flux densities, set at 5, 10,20,50,100, and 200 $\sigma$.

(A color version of this figure is available in the online journal)

The spectra were reduced using the $2 d f d r$ reduction package, developed by the AAO specifically for $2 \mathrm{dF}$ spectra data. We were unable to place a fiber on all targets due to crowding, so higher priority was given to our radio sources with bright (B $<23$ ) counterparts. A total of 268 sources from the $1.4 \mathrm{GHz}$ ATHDF-S catalog were targeted. Redshifts were determined by visually inspecting the spectra. Each spectrum was assigned an index, $Q$, that signifies the quality of the redshift determination. A value of $Q=3$ indicates that three or more lines were identified, so the redshift is very well determined. $Q=2,1$, or 0 , indicates two, one or no lines identified respectively. Redshifts were obtained for 98 out of the 268 targeted sources. In Figure 12, we plot $B$ magnitude against $1.4 \mathrm{GHz}$ flux density for the ATHDF-S radio sources. From this we can see that the 


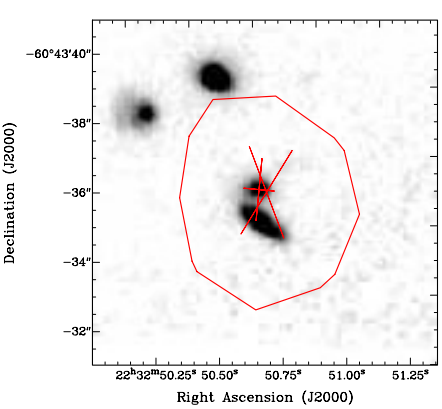

A T H D F S_J $223250.6-604336$

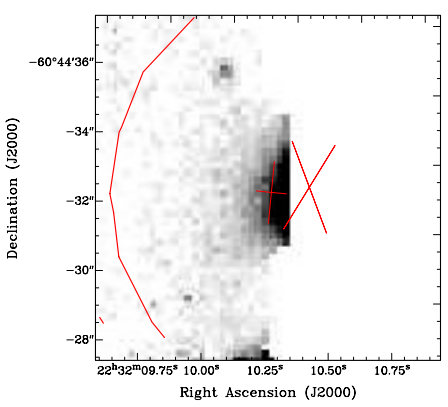

AT H D F S_J $223210.3-604433$

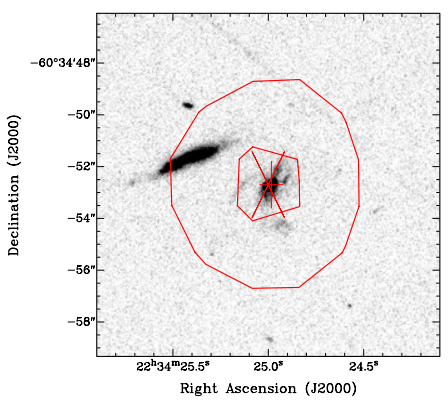

A T H D F S_J $223425.0-603452$

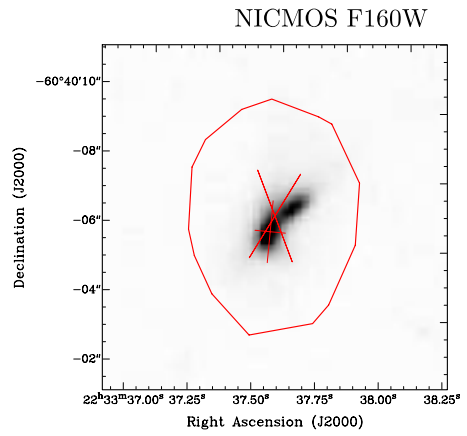

A T H D F S_J $223337.5-604006$

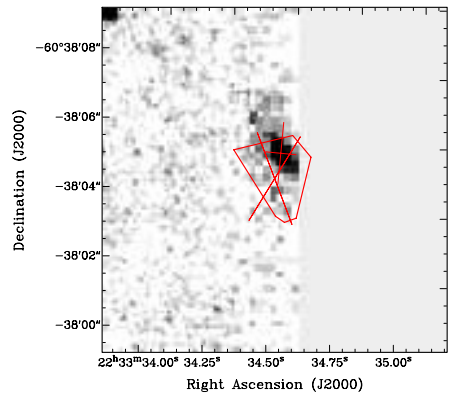

A T H D F S _J $223334.4-603804$

STIS 50CCD

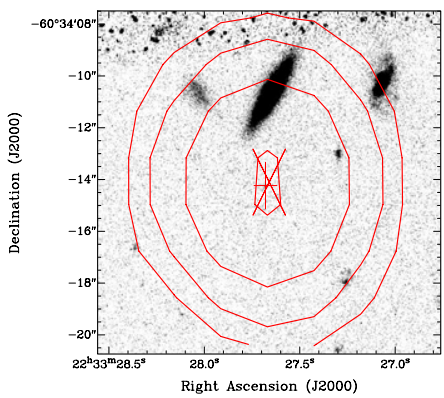

A T H D F S _J $223327.6-603414$

Figure 11. Gray-scale postage stamp images of the HST NICMOS and STIS flanking field counterparts to ATHDF-S radio sources. The crosses mark the radio position and the plus signs mark the optical positions. The contours are $1.4 \mathrm{GHz}$ flux densities, set at 5, 10, 20, 50, 100, and 200 $\sigma$.

(A color version of this figure is available in the online journal)

$2 \mathrm{dF}$ success rate is almost $100 \%$ for all targeted sources brighter than about $B=22$. Redshifts are much harder to obtain for the fainter sources. In the $B>23$ cases, the spectra are low $\mathrm{S} / \mathrm{N}$ and the redshift determination is only possible by the detection of bright emission lines.

The spectra were also used to classify our radio sources. We divided the spectra into five broad classes:

(i) galaxies with absorption line spectra $(22,22 \%)$,

(ii) star-forming galaxies $(36,37 \%)$,

(iii) Seyfert galaxies $(6,6 \%)$,

(iv) broadline AGNs (7, 7\%),

(v) unclassified objects $(27,28 \%)$.

The unclassified objects have at least one identifiable line in their spectrum, but insufficient $\mathrm{S} / \mathrm{N}$ or not enough observed lines to make a classification possible.

The Seyferts were determined by [O III] $5007 / \mathrm{H} \beta$ and [N II]6583/H $\alpha$ diagnostic line ratios. Although the $2 \mathrm{dF}$ spectrograph is fiber fed, these line pairs are close in wavelength and thus poor flux calibration will not affect these line ratios significantly. The line ratios of all our star-forming and Seyfert objects are plotted in Figure 13 along with the Kewley et al. (2001) classification line. The six Seyferts lie above and to the right of the maximum starburst line.

In Table 10 we present a summary of the $2 \mathrm{dF}$ spectroscopy of the ATHDF-S sources. Information is provided for the sources with $2 \mathrm{dF}$ redshifts. A description of Table 10 is as follows:

Column 1: ATHDF-S source name.

Column 2: $2 \mathrm{dF}$ spectroscopic redshift.

Column 3: quality of $2 \mathrm{dF}$ spectroscopy, as described above.

Column 4: spectral classification, as described above. "abs" are galaxies with absorption lines, "sf" are star-forming galaxies, "sy" indicates Seyferts, "BL" marks broadline AGNs, and "unc" means unclassified.

Column 5: $\mathrm{H} \beta$ line flux, in CCD counts.

Column 6: O III[5007] line flux, in CCD counts.

Column 7: $\mathrm{H} \alpha$ line flux, in CCD counts.

Column 8: N II[6584] line flux, in CCD counts.

Column 9: comments on spectra, including which lines are observed. 
Table 6

The $I$ Magnitudes of ATHDF-S Sources in the HST WFPC2 Deep and Flanking Fields

\begin{tabular}{|c|c|c|c|}
\hline ATHDF-S name & $\begin{array}{c}\text { CTIO I } \\
\text { magnitude }\end{array}$ & $\begin{array}{c}H S T \text { F812W } \\
\text { magnitude }\end{array}$ & $\begin{array}{c}S_{1.4 \mathrm{GHz}} \\
(\mathrm{mJy})\end{array}$ \\
\hline ATHDF-S_J223207.9-603928 & & 25.61 & 0.098 \\
\hline ATHDF-S_J223253.8-603921 & 20.68 & 21.12 & 0.052 \\
\hline ATHDF-S_J223245.6-603857 & 23.39 & 23.91 & 0.843 \\
\hline ATHDF-S_J223248.2-603805 & 20.49 & 20.90 & 0.076 \\
\hline ATHDF-S_J223254.5-603748 & 17.91 & 18.44 & 0.092 \\
\hline ATHDF-S_J223202.5-603714 & 23.60 & 24.17 & 0.148 \\
\hline ATHDF-S_J223253.0-603539 & 23.19 & 23.45 & 0.090 \\
\hline ATHDF-S_J223245.3-603537 & 23.77 & 24.04 & 0.051 \\
\hline ATHDF-S_J223338.8-603523 & 18.61 & 18.99 & 0.185 \\
\hline ATHDF-S_J223344.9-603515 & 18.69 & 19.51 & 0.344 \\
\hline ATHDF-S_J223350.5-603503 & 23.08 & 26.24 & 1.252 \\
\hline ATHDF-S_J223307.1-603448 & & 24.83 & 0.103 \\
\hline ATHDF-S_J223243.3-603443 & 18.87 & 19.32 & 0.063 \\
\hline ATHDF-S_J223245.5-603419 & 20.33 & 21.10 & 0.265 \\
\hline ATHDF-S_J223311.9-603417 & 23.09 & 23.93 & 0.059 \\
\hline ATHDF-S_J223306.0-603350 & 16.58 & 17.25 & 0.452 \\
\hline ATHDF-S_J223258.6-603346 & $\cdots$ & 25.12 & 1.010 \\
\hline ATHDF-S_J223243.4-603352 & 19.45 & 19.87 & 0.098 \\
\hline ATHDF-S_J223247.6-603337 & 19.16 & 19.55 & 0.075 \\
\hline ATHDF-S_J223337.5-603329 & 16.51 & 17.70 & 1.126 \\
\hline ATHDF-S_J223253.1-603329 & 22.29 & 22.56 & 0.113 \\
\hline ATHDF-S_J223303-603324 & 22.31 & 22.79 & 0.051 \\
\hline ATHDF-S_J223339.4-603306 & 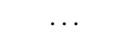 & 24.81 & 0.058 \\
\hline ATHDF-S_J223308.6-603251 & 20.45 & 21.03 & 0.821 \\
\hline ATHDF-S_J223317.7-603235 & 19.57 & 20.83 & 0.070 \\
\hline ATHDF-S_J223331.6-603222 & 19.90 & 20.38 & 0.395 \\
\hline ATHDF-S_J223303.1-603132 & $\ldots$ & 24.05 & 0.052 \\
\hline
\end{tabular}

Notes. The eight sources not detected by CTIO and HST are listed separately in Table 7.

Table 7

ATHDF-S Sources in the HST WFPC2 Deep and Flanking Fields which Remain Undetected to $I \sim 26.0$

\begin{tabular}{lcc}
\hline \hline ATHDF-S name & $S_{1.4 \mathrm{GHz}}(\mathrm{mJy})$ & Radio-to-optical ratio $R_{I}$ \\
\hline ATHDF-S_J223258.7-603903 & 0.058 & $>2.60$ \\
ATHDF-S_J223205.9-603857 & 0.254 & $>3.24$ \\
ATHDF-S_J223327.6-603414 & 0.456 & $>3.50$ \\
ATHDF-S_J223327.9-603304A & 0.221 & $>3.18$ \\
ATHDF-S_J223327.9-603304B & 0.059 & $>2.61$ \\
ATHDF-S_J223323.2-603249 & 0.457 & $>3.50$ \\
ATHDF-S_J223335.3-603234 & 0.054 & $>2.47$ \\
ATHDF-S_J223302.1-603213 & 0.063 & $>2.64$ \\
\hline
\end{tabular}

\section{PHOTOMETRIC REDSHIFTS}

The majority of our radio sources do not have spectral data, but CTIO imaging provides up to five-band photometry of $67 \%$ of the radio sources with which to calculate photometric redshifts. Photometric redshifts were determined using the galaxy template technique by two independent groups: Teplitz et al. (2001) and ourselves. Teplitz et al. (2001) used a template set which consisted of Coleman et al. (1980) spectra augmented with a single bluer synthetic starburst (Bruzual \& Charlot 1993). They calculated photometric redshifts for objects with a clear detection $(>3 \sigma)$ in $B V R$ and either $u$ or $I$. We adopted the template set of Coleman et al. (1980) spectra plus two starburst galaxy spectra. The two new starburst galaxy spectra were formed by adopting starbursts SB1 and SB2 of Kinney et al. (1996), which have different intrinsic color excess, and

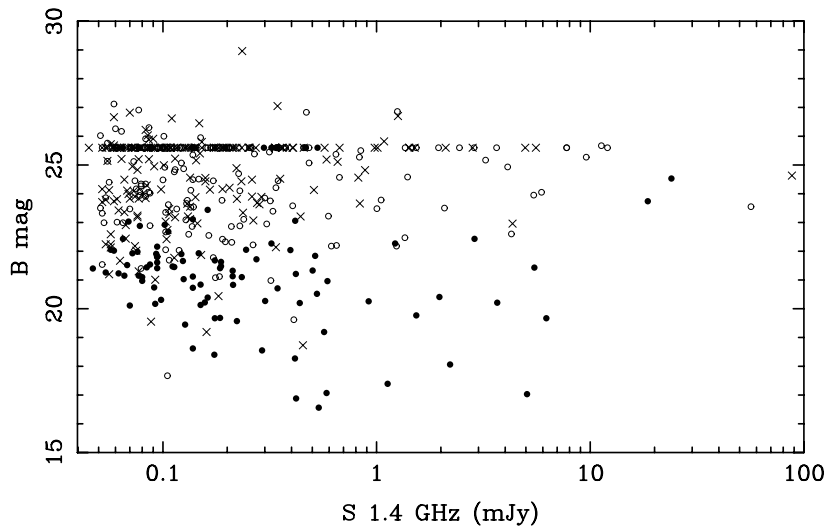

Figure 12. $B$-band magnitude versus $1.4 \mathrm{GHz}$ flux density for all ATHDF$S$ radio sources. The solid circles are sources with redshifts successfully obtained from $2 \mathrm{dF}$. The empty circles are $2 \mathrm{dF}$ targeted sources, but no redshift was determined. The crosses mark sources which were not targeted in the $2 \mathrm{dF}$ observations. Radio sources without optical counterparts are placed at $B=25.6$, the nominal $B$-band limit of the CTIO observations. We note that in two cases with $B<20$ no redshift was recorded. The first case is ATHDF-S_J223222.4-602532, which is near a star, so the $B$ magnitude of 17.67 is probably overestimated. We suspect that the fiber was incorrectly placed for the second case (ATHDF-S_J223414.7-604753).

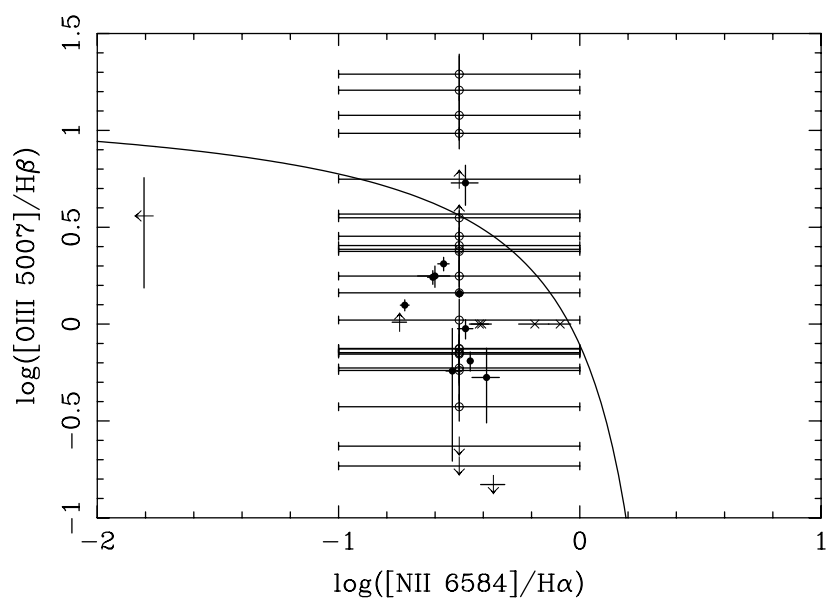

Figure 13. Diagnostic line ratio $[\mathrm{N}$ II $] 6583 / \mathrm{H} \alpha$ against $[\mathrm{O}$ III $] 5007 / \mathrm{H} \beta$ for the 42 narrowline emitting galaxies in our $2 \mathrm{dF}$ sample. The solid circles indicate sources where all four lines are detected. The open circles mark high redshift galaxies where the $\mathrm{H} \alpha$ and $\mathrm{N}$ II lines are out of the wavelength coverage. For these sources the uncertainty in [N II] $6583 / \mathrm{H} \alpha$ is marked by large error bars. The crosses mark sources with no detectable $\mathrm{H} \beta$ or [O III] 5007 emission. The arrows indicate upper and lower limits. The solid curve is the theoretical maximum starburst line from Kewley et al. (2001). Star-forming galaxies lie below this line, while AGNs are found above this line.

extrapolating to ultraviolet and near-IR wavelengths using the recipe from Fernández-Soto et al. (1999).

Teplitz et al. (2001) performed a Monte Carlo simulation to estimate the accuracy of their photometric redshifts. Model galaxy spectra (Bruzual \& Charlot 1993) of varying metallicity and age were redshifted and random photometric error added. The redshift error from applying the photometric redshift method to the simulated spectra is typically $15-20 \%$ for $z<1$ (Teplitz et al. 2001). For galaxies with $z>1$ the typical residuals in the redshift error are $\Delta z>0.35$. Also, the failures $(\Delta z>0.33)$ for $z<1$ simulated galaxies are less than $10 \%$, compared with greater than $40 \%$ for $z>1.33$ objects. The failures in the Teplitz et al. (2001) photometric redshift catalog are likely to 
Table 8

Summary of LDSS++ Spectra (Glazebrook et al. 2006) of ATHDF-S Radio Sources

\begin{tabular}{lcccc}
\hline \hline ATHDF-S name & $\begin{array}{c}\text { LDSS++ } \\
\text { number }\end{array}$ & Redshift & Spectral type & Comments \\
\hline ATHDF-S_J223241.4-603025 & 216 & 0.4250 & abs & O II, Balmer-, HK, H $\beta+$ \\
ATHDF-S_J223247.6-603337 & 196 & 0.5803 & abs & O II, HK, H $\delta$-, G, H $\gamma-$ \\
ATHDF-S_J223306.0-603350 & 133 & 0.1733 & sf & $\mathrm{H} \beta+$, O III+, Mgb, 5268, H $\alpha+$, N II+, S II+ \\
ATHDF-S_J223245.5-603419 & 204 & 0.4606 & sf & H $\beta+$, O III(4959+5007)+ \\
\hline
\end{tabular}

Table 9

Summary of FORS2 Spectra (Sawicki \& Mallén-Ornelas 2003) of ATHDF-S Radio Sources

\begin{tabular}{lcc}
\hline \hline ATHDF-S name & $\begin{array}{c}\text { Sawicki \& Mallén-Ornelas (2003) } \\
\text { ID }\end{array}$ & Redshift \\
& Comments \\
\hline ATHDF-S_J223254.5-603748 & 90549 & 0.2668 \\
ATHDF-S_J223243.3-603443 & 20277 & 0.4233 \\
ATHDF-S_J223245.5-603419 & 20462 & 0.4608 \\
ATHDF-S_J223247.6-603337 & 672 & 0.5807 \\
ATHDF-S_J223302.8-603323 & 894 & 0.4642 \\
\hline
\end{tabular}

be more numerous, since real data contain a larger variety of galaxies, such as AGNs. Nevertheless, one can conclude that their photometric redshifts are quite reliable to $z=1$. The reason for the failure at $z>1$ is the limitations of the CTIO filter set. The most obvious spectral features for template fitting are the $4000 \AA$ and $912 \AA$ breaks, which fall out of the $u B V R I$ passbands. The $4000 \AA$ is shifted to near-IR for $z>1$, while the $912 \AA$ is out of the $u$ passband until $z \sim 2.5$. The CTIO photometric redshifts are listed in Table 11.

We expect our photometric redshifts to have similar accuracy, since similar data and techniques are used. A photometric redshift was estimated for the $3071.4 \mathrm{GHz}$ radio sources with a CTIO counterpart from the CTIO imaging. The photometric redshifts are restricted to $0<z<1$, as this is the redshift space effectively probed by the $u B V R I$ filter set. Photometric redshifts of $z=0$ and $z=1$ were rejected since the photometric redshift code obviously did not find a fit within the desired range. This occurred for 43 of the sources, so in total we derive 264 photometric redshifts. We list these in Table 11.

To investigate the accuracy of our photometric redshifts we compared them with the spectroscopic ones obtained on $2 \mathrm{dF}$. Of the 98 objects with $2 \mathrm{dF}$ redshifts, 85 have photometric redshifts. We show a comparison of the $2 \mathrm{dF}$ spectroscopic versus photometric redshifts in the left panel of Figure 14. We find that only six ATHDF-S sources have estimated photometric redshifts which are failures $\left(\delta z /\left(1+z_{\text {spec }}\right)>0.2\right)$, indicating a high photometric redshift success rate of 79/85 (93\%). Four of these sources are broadline emitting quasars which are not well represented by our galaxy templates. The 79 successful photometric redshifts have a median residual $\delta z /\left(1+z_{\text {spec }}\right)$ of $0.008 \pm 0.064$. In comparison, there are photometric redshifts from Teplitz et al. (2001) for 87 of the sources with $2 \mathrm{dF}$ redshifts. A comparison of Teplitz et al. (2001) and $2 \mathrm{dF}$ redshifts is shown in the right panel of Figure 14. The success rate $\left(\delta z /\left(1+z_{\text {spec }}\right)<0.2\right)$ of the Teplitz et al. (2001) photometric redshifts is $72 / 87$ (83\%). We note that there are eight sources with a spectroscopic redshift $z<1$ which are erroneously given photometric redshifts $z>1$ by Teplitz et al. (2001). Also, even though Teplitz et al. (2001) allow photometric redshifts greater than $z=1$, they successfully estimate the redshift of only $1 / 6$ of the broadline emitting objects at $z>1$. We therefore deem our photometric redshifts to be more reliable than Teplitz et al. (2001) and use only our photometric redshifts in further analysis.
The photometric redshifts are accurate for the $2 \mathrm{dF}$ sample. However, is this likely to be true for the full ATHDF-S sample? The sources with $2 \mathrm{dF}$ redshifts are the optically brightest of our sources (see Figure 12), so the previous comparison between photometric and spectroscopic redshifts is not from a random selection of the full ATHDF-S sample. To estimate the contamination in the photometric redshifts we can combine the results from Teplitz et al. (2001) with ours. Figure 15 shows the comparison of the 209 sources which also have photometric redshifts from Teplitz et al. (2001). In the cases where Teplitz et al. (2001) $z<1$, which is $185 / 209(89 \%)$, there is good agreement, and the median $\delta z /\left(1+z_{\text {phot }}\right)$ is $-0.046 \pm 0.097$. The two photometric redshift samples are independent fits, yet they agree $\left(\delta z /\left(1+z_{\text {phot }}\right)<0.2\right)$ for $\sim 87 \%$ of the sources with Teplitz et al. (2001) redshifts. By design, our photometric redshifts will be wrong for sources that actually lie at $z>1$, but from Figure 15 we see that sources which are possibly at $z>1$ (from Teplitz et al. 2001) have a flat distribution in our photometric redshifts, and hence are not biased to a particular redshift. It is not clear whether the Teplitz et al. (2001) redshifts are overestimated or our redshifts underestimated for the discordant cases. However, it is likely that our results are correct for a significant portion of these discordant cases since the Teplitz et al. (2001) results have a $40 \%$ failure rate for $z>1$.

The photometric redshifts of our ATHDF-S radio sources are listed in Table 11. A description of the table is as follows:

Column 1: ATHDF-S source name.

Column 2: Teplitz et al. (2001) photometric redshift.

Column 3: photometric redshift from this work.

Column 4: best-fit spectral template, as described above.

\section{DISCUSSION}

\subsection{Optical Properties of the Sub-mJy Sample}

The magnitude distributions of our matched radio sources, at all five CTIO optical bands, are shown in Figure 16. The $R$ magnitude distribution shows that the number of sources declines between $R=22$ and $R=24$, and there are similar decreases in the other passbands. We argue that this is a property of the CTIO-identified ATHDF-S radio sources, rather than being caused by incompleteness in the optical imaging, since the drop-off in number occurs well above the limiting magnitude of 
Table 10

Summary of 2dF Spectral Properties for ATHDF-S Sources with 2dF Redshifts

\begin{tabular}{|c|c|c|c|c|c|c|c|c|}
\hline ATHDF-S name & $\begin{array}{c}2 \mathrm{dF} \\
\text { redshift }\end{array}$ & $\begin{array}{c}2 \mathrm{dF} \\
\text { quality }\end{array}$ & $\begin{array}{l}\text { Spectral } \\
\text { type }\end{array}$ & $\begin{array}{c}\mathrm{H} \beta \\
\text { line flux }\end{array}$ & $\begin{array}{l}\text { O III [5007] } \\
\text { line flux }\end{array}$ & $\begin{array}{c}\mathrm{H} \alpha \\
\text { line flux }\end{array}$ & $\begin{array}{c}\text { N II [6584] } \\
\text { line flux }\end{array}$ & Comments \\
\hline ATHDFS_J223356.6-601949 & 0.2856 & 3 & abs & $\ldots$ & $\ldots$ & $\ldots$ & $\ldots$ & OII, HK, G, Mg \\
\hline ATHDFS_J223358.9-602258 & 2.519 & 3 & BL & $\ldots$ & $\ldots$ & $\ldots$ & $\ldots$ & La, OIV], CIV and CIII] \\
\hline ATHDFS_J223134.5-602457 & 0.5636 & 2 & unc & $\ldots$ & $\ldots$ & $\ldots$ & $\ldots$ & OII, HK \\
\hline ATHDFS_J223141.1-602506 & 0.2485 & 3 & sf & $1180 \pm 230$ & $680 \pm 210$ & $\ldots$ & $\ldots$ & OII, HK, Hb, OIII \\
\hline ATHDFS_J223136.9-602610 & 0.5189 & 2 & unc & $\ldots$ & $\ldots$ & $\ldots$ & $\ldots$ & OII, H, K \\
\hline ATHDFS_J223335.3-602615 & 0.1726 & 3 & sf & $<70$ & $<70$ & $3860 \pm 230$ & $3200 \pm 280$ & OII, HK, Ha, N2 \\
\hline ATHDFS_J223400.9-602633 & 0.4314 & 3 & abs & $\ldots$ & $\ldots$ & $\ldots$ & $\ldots$ & $\mathrm{H}, \mathrm{K}, \mathrm{G}, \mathrm{Mg}$ \\
\hline ATHDFS_J223418.3-602715 & 0.3034 & 1 & unc & $\ldots$ & $\ldots$ & $\ldots$ & $\ldots$ & OII \\
\hline ATHDFS_J223311.5-602725 & 0.1084 & 3 & sf & $<130$ & $<130$ & $9050 \pm 470$ & $3470 \pm 260$ & OII, HK, G, weak Hb, weak OIII, Ha, N2, S2 \\
\hline ATHDFS_J223443.9-602739B & 0.4415 & 2 & abs & $\ldots$ & $\ldots$ & $\ldots$ & $\ldots$ & $\mathrm{HK}, \mathrm{G}$ \\
\hline ATHDFS_J223330.5-602849 & 0.4149 & 3 & sf & $1730 \pm 130$ & $6120 \pm 280$ & $\ldots$ & $\ldots$ & OII, Hb, OIII \\
\hline ATHDFS_J223515.2-602856 & 0.4424 & 3 & sf & $350 \pm 90$ & $890 \pm 170$ & $\ldots$ & $\ldots$ & OII, HK, Hb, OIII \\
\hline ATHDFS_J223431.7-602859 & 0.4275 & 3 & sf & $640 \pm 130$ & $<150$ & - & $\ldots$ & OII, H, K, Hb \\
\hline ATHDFS_J223415.3-602925 & 0.1679 & 3 & sf & $<100$ & $<100$ & $4870 \pm 330$ & $3170 \pm 400$ & OII, HK, G, Hb, OIII, Ha, N2, S2 \\
\hline ATHDFS_J223108.1-602946 & 0.5646 & 1 & unc & $\ldots$ & $\ldots$ & $\ldots$ & $\ldots$ & OII \\
\hline ATHDFS_J223534.2-602948 & 0.1752 & 3 & sf & $<50$ & $<50$ & $5050 \pm 260$ & $1980 \pm 160$ & OII, Mg, Na, Ha, N2 \\
\hline ATHDFS_J223440.4-603017 & 0.3251 & 3 & abs & $\ldots$ & $\ldots$ & $\ldots$ & $\ldots$ & $\mathrm{HK}, \mathrm{Hb}$ abs, G, Mg, \\
\hline ATHDFS_J223120.1-603025 & 0.4239 & 2 & unc & $\ldots$ & $\ldots$ & $\ldots$ & $\ldots$ & OII, $\mathrm{H}$ \\
\hline ATHDFS_J223304.8-603031 & 0.4282 & 3 & unc & $\ldots$ & $\ldots$ & $\ldots$ & $\ldots$ & OII, Hb, OIII \\
\hline ATHDFS_J223504.6-603109 & 0.0587 & 3 & sf & $6250 \pm 460$ & $12800 \pm 400$ & $35700 \pm 800$ & $9730 \pm 480$ & OII, HK, G, Hb, OIII, Ha, N2, S2 \\
\hline ATHDFS_J223519.4-603157A & 0.4081 & 3 & sf & $2030 \pm 220$ & $1510 \pm 230$ & $\ldots$ & $\ldots$ & OII, Hb, OIII \\
\hline ATHDFS_J223519.4-603157B & 0.4067 & 3 & sf & $800 \pm 80$ & $570 \pm 70$ & $\ldots$ & $\ldots$ & OII, HB, OIII \\
\hline ATHDFS_J223343.5-603211 & 0.5302 & 3 & sf & $370 \pm 110$ & $878 \pm 150$ & $\ldots$ & $\ldots$ & OII, HK, Hb, OIII \\
\hline ATHDFS_J223504.4-603221 & 0.4265 & 1 & unc & $\ldots$ & $\ldots$ & $\ldots$ & $\ldots$ & OIII \\
\hline ATHDFS_J223331.6-603222 & 0.4652 & 3 & sf & $650 \pm 120$ & $1150 \pm 100$ & $\ldots$ & $\ldots$ & OII, Hb, OIII \\
\hline ATHDFS_J223509.4-603235 & 0.1200 & 3 & abs & $\ldots$ & $\ldots$ & $\ldots$ & $\ldots$ & $\mathrm{H}, \mathrm{K}, \mathrm{Hb}, \mathrm{OIII}, \mathrm{Mg}, \mathrm{Na}$ \\
\hline ATHDFS_J223142.5-603238 & 0.3445 & 2 & abs & $\ldots$ & $\ldots$ & $\ldots$ & $\ldots$ & $\mathrm{H}, \mathrm{K}, \mathrm{Mg}$ \\
\hline ATHDFS_J223337.5-603329 & 2.238 & 3 & BL & $\ldots$ & $\ldots$ & $\ldots$ & $\ldots$ & STIS QSO z $=2.238$ \\
\hline ATHDFS_J223513.7-603333 & 0.4070 & 2 & sf & $810 \pm 150$ & $<150$ & $\ldots$ & $\ldots$ & OII, HB \\
\hline ATHDFS_J223225.0-603338 & 0.2998 & 2 & sf & $\ldots$ & $1210 \pm 120$ & - & $\ldots$ & OII, OIII \\
\hline ATHDFS_J223243.4-603352 & 1.566 & 3 & BL & $\ldots$ & $\ldots$ & $\ldots$ & $\ldots$ & $\mathrm{CIV}, \mathrm{CIII}]$ and $\mathrm{MgII}$ \\
\hline ATHDFS_J223401.0-603424 & 0.2832 & 2 & abs & $\ldots$ & $\ldots$ & $\ldots$ & $\ldots$ & $\mathrm{HK}, \mathrm{Mg}, \mathrm{G}$ \\
\hline ATHDFS_J223442.8-603433 & 0.0668 & 3 & sf & $680 \pm 390$ & $2460 \pm 110$ & $1920 \pm 140$ & $<30$ & OII, Hb, OIII, Ha \\
\hline ATHDFS_J223212.3-603448 & 0.1823 & 2 & unc & $\ldots$ & $\ldots$ & $\ldots$ & $\ldots$ & OII, OIII \\
\hline ATHDFS_J223438.6-603450 & 0.3354 & 3 & abs & $\ldots$ & $\ldots$ & $\ldots$ & $\ldots$ & $\mathrm{HK}, \mathrm{G}, \mathrm{Hb}$ abs, $\mathrm{Mg}$ \\
\hline ATHDFS_J223541.5-603454 & 0.6142 & 1 & unc & $\ldots$ & $\ldots$ & $\ldots$ & $\ldots$ & OII \\
\hline ATHDFS_J223344.9-603515 & 0.5273 & 3 & sf & $2510 \pm 230$ & $940 \pm 120$ & $\ldots$ & $\ldots$ & OII, Hb, OIII \\
\hline ATHDFS_J223208.3-603519 & 0.2841 & 3 & sy & $600 \pm 160$ & $11700 \pm 400$ & $\ldots$ & $\ldots$ & OII, HK, OIII \\
\hline ATHDFS_J223338.8-603523 & 0.2250 & 3 & abs & $\ldots$ & $\ldots$ & $\ldots$ & $\ldots$ & $\mathrm{H}, \mathrm{K}, \mathrm{G}, \mathrm{OIII}$ \\
\hline ATHDFS_J223046.1-603525 & 0.3002 & 3 & $\mathrm{sf}$ & $2200 \pm 210$ & $6250 \pm 260$ & $\ldots$ & $\ldots$ & OII, Hb, OIII \\
\hline ATHDFS_J223232.4-603542A & 0.4338 & 2 & abs & $\ldots$ & $\ldots$ & $\ldots$ & $\ldots$ & $\mathrm{HK}, \mathrm{G}$ \\
\hline ATHDFS_J223232.4-603542B & 0.4338 & 2 & abs & $\ldots$ & $\ldots$ & $\cdots$ & $\ldots$ & HK, G \\
\hline ATHDFS_J223525.7-603544 & 0.0586 & 3 & sf & $3320 \pm 380$ & $5880 \pm 310$ & $19700 \pm 700$ & $4940 \pm 730$ & OII, Hb, OIII, Ha, N2, S2 \\
\hline ATHDFS_J223104.8-603549 & 0.5693 & 1 & unc & $\ldots$ & $\ldots$ & $\ldots$ & $\ldots$ & OII \\
\hline ATHDFS_J223421.8-603603 & 0.3499 & 3 & sf & $3840 \pm 250$ & $2280 \pm 340$ & - & $\ldots$ & OII, Hb, OIII \\
\hline ATHDFS_J223158.5-603614 & 0.4213 & 2 & unc & $\ldots$ & $\ldots$ & $\ldots$ & $\ldots$ & OII, K \\
\hline ATHDFS_J223451.7-603632 & 0.3291 & 3 & sf & $900 \pm 110$ & $640 \pm 190$ & $\ldots$ & $\ldots$ & OII, H, K, Hb, OIII \\
\hline ATHDFS_J223406.7-603637 & 0.0991 & 3 & sf & $3750 \pm 300$ & $3550 \pm 290$ & $21200 \pm 400$ & $7120 \pm 500$ & OII, HK, Hb, OIII, Na, Ha, N2, S2 \\
\hline ATHDFS_J223400.2-603653 & 0.0992 & 3 & sf & $7070 \pm 480$ & $4560 \pm 410$ & $64600 \pm 820$ & $22700 \pm 560$ & OII, HK, Hb, OIII, Na, Ha, N2, S2 \\
\hline ATHDFS_J223417.1-603724 & 0.2403 & 3 & abs & $\ldots$ & $\ldots$ & $\ldots$ & $\ldots$ & OII, HK, G, Mg, some OIII \\
\hline ATHDFS_J223437.7-603726 & 0.4137 & 3 & sf & $4420 \pm 160$ & $3310 \pm 310$ & $\ldots$ & $\ldots$ & OII, Hb, OIII \\
\hline ATHDFS_J223254.5-603748 & 0.1798 & 3 & sy & $840 \pm 190$ & $4500 \pm 260$ & $5420 \pm 210$ & $1820 \pm 220$ & OII, H, K, Hb, OIII, Ha, N2 \\
\hline ATHDFS_J223418.6-603808 & 0.4279 & 3 & sy & $2010 \pm 140$ & $19400 \pm 200$ & - & $\ldots$ & OII, Hb, OIII \\
\hline ATHDFS_J223450.4-603844 & 0.4180 & 2 & abs & $\ldots$ & $\ldots$ & $\ldots$ & $\ldots$ & $\mathrm{K}, \mathrm{G}$ \\
\hline ATHDFS_J223153.7-603853 & 0.7500 & 1 & unc & $\ldots$ & $\ldots$ & $\ldots$ & $\ldots$ & OII \\
\hline ATHDFS_J223144.4-603858 & 0.3102 & 3 & abs & $\ldots$ & $\ldots$ & $\ldots$ & $\ldots$ & $\mathrm{H}, \mathrm{K}, \mathrm{G}, \mathrm{Hb}, \mathrm{Mg}$ \\
\hline ATHDFS_J223123.1-603903 & 0.2782 & 1 & unc & $\ldots$ & $\ldots$ & $\ldots$ & $\ldots$ & OII \\
\hline ATHDFS_J223222.7-603924 & 0.4215 & 2 & unc & $\ldots$ & $\ldots$ & $\ldots$ & $\ldots$ & OII, K, G \\
\hline ATHDFS_J223220.6-603931 & 0.2825 & 3 & abs & $\ldots$ & $\ldots$ & $\ldots$ & $\ldots$ & $\mathrm{HK}, \mathrm{G}, \mathrm{Na}, \mathrm{Mg}$ \\
\hline ATHDFS_J223337.5-604006 & 0.425 & 3 & sf & $530 \pm 110$ & $1290 \pm 140$ & $\ldots$ & $\ldots$ & OII, Hb, OIII \\
\hline ATHDFS_J223448.3-604042 & 0.1463 & 3 & sf & $<4000$ & $4090 \pm 280$ & $18600 \pm 500$ & $3330 \pm 210$ & OII, HK, OIII, Ha \\
\hline ATHDFS_J223255.4-604151 & 1.233 & 2 & BL & $\ldots$ & $\ldots$ & $\ldots$ & $\ldots$ & MgII, CIII] \\
\hline ATHDFS_J223359.8-604155 & 0.0582 & 3 & abs & $\ldots$ & $\ldots$ & $\ldots$ & $\ldots$ & OII, H, K, G, Mg, Ha, N2 \\
\hline ATHDFS_J223437.2-604214 & 0.4645 & 2 & unc & $\ldots$ & $\ldots$ & $\ldots$ & $\ldots$ & OII, HK \\
\hline ATHDFS_J223338.8-604216 & 0.3113 & 3 & sf & $5690 \pm 200$ & $8250 \pm 350$ & $\ldots$ & - & OII, HK, Hb, OIII \\
\hline
\end{tabular}


Table 10

(Continued)

\begin{tabular}{|c|c|c|c|c|c|c|c|c|}
\hline ATHDF-S name & $\begin{array}{c}2 \mathrm{dF} \\
\text { redshift }\end{array}$ & $\begin{array}{c}2 \mathrm{dF} \\
\text { quality }\end{array}$ & $\begin{array}{l}\text { Spectral } \\
\text { type }\end{array}$ & $\begin{array}{c}\mathrm{H} \beta \\
\text { line flux }\end{array}$ & $\begin{array}{l}\text { O III [5007] } \\
\text { line flux }\end{array}$ & $\begin{array}{c}\mathrm{H} \alpha \\
\text { line flux }\end{array}$ & $\begin{array}{l}\text { N II [6584] } \\
\text { line flux }\end{array}$ & Comments \\
\hline ATHDFS_J223312.4-604227 & 0.5244 & 1 & unc & $\ldots$ & $\ldots$ & $\ldots$ & $\ldots$ & OII \\
\hline ATHDFS_J223207.9-604305 & 0.7570 & 2 & unc & $\ldots$ & $\ldots$ & $\ldots$ & $\ldots$ & OII, HK \\
\hline ATHDFS_J223240.2-604306 & 0.2982 & 2 & sy & $<2000$ & $11200 \pm 600$ & $\ldots$ & - & OII, Hb, OIII, HK \\
\hline ATHDFS_J223430.8-604310 & 0.3394 & 2 & sf & $<200$ & $740 \pm 180$ & - & $\ldots$ & OII, OIII \\
\hline ATHDFS_J223440.4-604309 & 0.3124 & 2 & unc & $\ldots$ & $\ldots$ & $\ldots$ & $\ldots$ & OII, H, K \\
\hline ATHDFS_J223522.2-604326 & 0.1772 & 3 & sf & $4830 \pm 360$ & $2560 \pm 1050$ & $5600 \pm 490$ & $2300 \pm 220$ & OII, Hb, Ha, N2, HK \\
\hline ATHDFS_J223126.2-604337 & 0.0864 & 3 & sf & $5500 \pm 350$ & $9600 \pm 480$ & $27100 \pm 500$ & $6660 \pm 300$ & OII, OIII, $\mathrm{Hb}, \mathrm{Ha}, \mathrm{N} 2, \mathrm{~S} 2$ \\
\hline ATHDFS_J223313.9-604359 & 0.1758 & 3 & sf & $1010 \pm 130$ & $<150$ & $7530 \pm 270$ & $3300 \pm 360$ & OII, H, K, G, Hb, Ha, N2, S2 \\
\hline ATHDFS_J223319.1-604428 & 0.6465 & 2 & BL & $\ldots$ & $\ldots$ & $\ldots$ & $\ldots$ & \\
\hline ATHDFS_J223121.4-604448 & 0.6023 & 1 & unc & $\ldots$ & $\ldots$ & $\ldots$ & $\ldots$ & OII \\
\hline ATHDFS_J223443.3-604452 & 3.1 & 2 & BL & $\ldots$ & $\ldots$ & $\ldots$ & $\ldots$ & \\
\hline ATHDFS_J223342.9-604524 & 0.2454 & 3 & sy & $480 \pm 240$ & $7740 \pm 350$ & $\ldots$ & - & OII, OIII, HK, weak Hb \\
\hline ATHDFS_J223536.8-604632 & 0.1775 & 3 & sf & $3790 \pm 480$ & $2170 \pm 1400$ & $25900 \pm 500$ & $7670 \pm 420$ & $\mathrm{Hb}, \mathrm{Ha}$, low OIII, N2, OII \\
\hline ATHDFS_J223527.8-604639A & 0.4649 & 2 & abs & $\ldots$ & $\ldots$ & $\ldots$ & $\ldots$ & $\mathrm{H}, \mathrm{K}$ \\
\hline ATHDFS_J223333.2-604642 & 0.1393 & 3 & abs & $\ldots$ & $\ldots$ & $\ldots$ & $\ldots$ & abs $\mathrm{HK}, \mathrm{Na}$ \\
\hline ATHDFS_J223228.5-604642 & 0.6370 & 1 & unc & $\ldots$ & $\ldots$ & $\ldots$ & $\ldots$ & $\mathrm{H}, \mathrm{K}, \mathrm{G}$ \\
\hline ATHDFS_J223417.5-604749 & 0.2015 & 3 & sf & $600 \pm 130$ & $420 \pm 150$ & - & $\ldots$ & OII, H, K, Hb, OIII, Ha, N2 \\
\hline ATHDFS_J223217.6-604751 & 0.7103 & 1 & unc & $\ldots$ & $\ldots$ & $\ldots$ & $\ldots$ & OII \\
\hline ATHDFS_J223243.0-604758 & 0.5810 & 2 & unc & $\ldots$ & $\ldots$ & $\ldots$ & $\ldots$ & $\mathrm{Hb}$, OIII but lines in red skylines \\
\hline ATHDFS_J223452.0-604834 & 0.2461 & 2 & unc & $\ldots$ & $\ldots$ & $\ldots$ & $\ldots$ & OII, no $\mathrm{Hb}$ or OIII \\
\hline ATHDFS_J223337.8-604833 & 0.5121 & 1 & unc & $\ldots$ & $\ldots$ & $\ldots$ & $\ldots$ & OII \\
\hline ATHDFS_J223413.1-604909 & 0.2653 & 3 & abs & $\ldots$ & $\ldots$ & $\ldots$ & $\ldots$ & $\mathrm{OII}, \mathrm{HK}, \mathrm{Hb}$ \\
\hline ATHDFS_J223226.9-605029 & 0.4015 & 1 & unc & $\ldots$ & $\ldots$ & $\ldots$ & $\ldots$ & OII \\
\hline ATHDFS_J223139.6-605039 & 0.2834 & 3 & abs & $\ldots$ & $\cdots$ & $\ldots$ & $\ldots$ & $\mathrm{H}, \mathrm{K}, \mathrm{G}, \mathrm{Hb}, \mathrm{Mg}$ \\
\hline ATHDFS_J223352.5-605210 & 0.2540 & 1 & unc & $\ldots$ & $\ldots$ & $\ldots$ & $\ldots$ & OII \\
\hline ATHDFS_J223454.9-605211 & 0.0557 & 3 & sf & $47500 \pm 1100$ & $68300 \pm 800$ & $317400 \pm 2200$ & $100600 \pm 1700$ & OII, Hb, OIII, Ha, N2, S2 \\
\hline ATHDFS_J223145.4-605311 & 0.3140 & 3 & abs & $\ldots$ & $\ldots$ & $\ldots$ & $\ldots$ & strong $\mathrm{HK}, \mathrm{Hb}$ abs, $\mathrm{Mg}, \mathrm{Na}$ \\
\hline ATHDFS_J223151.1-605328 & 0.3114 & 3 & sy & $1630 \pm 420$ & $19500 \pm 400$ & - & $\ldots$ & OII, OIII, $\mathrm{Hb}, \mathrm{HK}, \mathrm{Na}$ \\
\hline ATHDFS_J223204.8-605414 & 0.2817 & 3 & sf & $1010 \pm 200$ & $1060 \pm 210$ & $\ldots$ & $\ldots$ & OII, H, K, Hb, OIII \\
\hline ATHDFS_J223214.8-605430 & 1.073 & 2 & BL & $\ldots$ & $\ldots$ & $\ldots$ & $\ldots$ & MgII, OII and CII \\
\hline ATHDFS_J223316.9-605533 & 0.0553 & 3 & abs & $\ldots$ & $\ldots$ & $\ldots$ & $\ldots$ & strong abs $\mathrm{HK}, \mathrm{Hb}, \mathrm{Mg}, \mathrm{Na}$ \\
\hline ATHDFS_J223314.6-605543 & 0.0563 & 3 & sf & $3170 \pm 140$ & $3970 \pm 190$ & $20300 \pm 500$ & $3820 \pm 130$ & OII, H, K, Hb, OIII, Ha, N2, S2 \\
\hline ATHDFS_J223303.6-605751 & 0.2202 & 2 & unc & $\ldots$ & $\ldots$ & $\ldots$ & $\ldots$ & OII, no $\mathrm{Hb}$ or OIII \\
\hline
\end{tabular}

(This table is also available in a machine-readable form in the online journal)
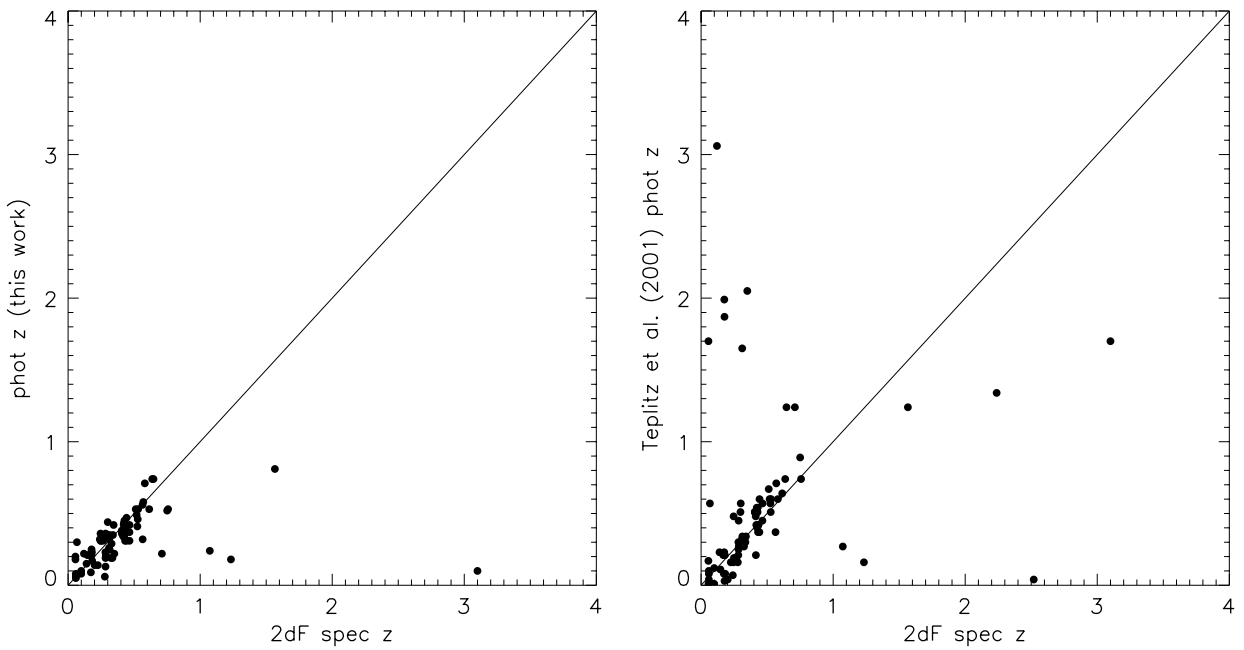

Figure 14. Left: comparison of $2 \mathrm{dF}$ spectroscopic and our photometric redshifts for the 85 ATHDF-S sources that have both. Right: comparison of $2 \mathrm{dF}$ spectroscopic and Teplitz et al. (2001) photometric redshifts for the 88 ATHDF-S sources that have both.

the optical data. The mean magnitude of the optical counterparts to our radio sources is $22.7,23.0,22.2,21.3$, and 20.4 for the $U-, B-, V-, R-$, and $I$-bands, respectively, whereas the limiting magnitude of the CTIO images are 24.0, 25.6, 25.0, 25.0, and 23.5 for the same passbands.
In Figure 17, we plot the $I$-band magnitude of the sample against $1.4 \mathrm{GHz}$ flux density. The lower flux density sources seem to have fewer bright $(I$ mag $<19)$ optical counterparts. The median $I$ magnitude for sources with $S_{1.4 \mathrm{GHz}}>0.5 \mathrm{mJy}$ is 0.8 mag brighter than the median $I$ magnitude of the faintest 
Table 11

Photometric Redshifts of ATHDF-S Radio Sources

\begin{tabular}{|c|c|c|c|}
\hline ATHDF-S name & Teplitz et al. (2001) redshift & Redshift (this work) & Spectral type \\
\hline ATHDFS_J223316.9-605533 & 0.17 & 0.20 & Sbc \\
\hline ATHDFS_J223353.9-605452 & 0.98 & 0.75 & Scd \\
\hline ATHDFS_J223214.8-605430 & 0.27 & 0.24 & Scd \\
\hline ATHDFS_J223448.4-605417 & 0.71 & 0.80 & Scd \\
\hline ATHDFS_J223204.8-605414 & 0.21 & 0.33 & Scd \\
\hline ATHDFS_J223230.3-605352 & 0.82 & 0.85 & $\mathrm{Sbc}$ \\
\hline ATHDFS_J223151.1-605328 & 0.32 & 0.25 & E/S0 \\
\hline ATHDFS_J223145.4-605311 & 0.32 & 0.25 & E/S0 \\
\hline ATHDFS_J223453.4-605259 & 0.74 & 0.74 & E/S0 \\
\hline ATHDFS_J223203.0-605242B & 0.34 & 0.27 & E/S0 \\
\hline ATHDFS_J223458.6-605225 & 0.71 & 0.71 & E/S0 \\
\hline ATHDFS_J223345.4-605227 & 1.44 & 0.48 & Irr \\
\hline ATHDFS_J223454.9-605211 & 1.70 & 0.18 & Scd \\
\hline ATHDFS_J223241.8-605209 & 0.10 & 0.11 & E/S0 \\
\hline ATHDFS_J223223.3-605137 & 0.51 & 0.37 & E/S0 \\
\hline ATHDFS_J223323.8-605141 & 0.51 & 0.68 & Scd \\
\hline ATHDFS_J223420.1-605138 & 0.82 & 0.84 & $\mathrm{Sbc}$ \\
\hline ATHDFS_J223427.0-605132 & 0.51 & 0.38 & Scd \\
\hline ATHDFS_J223238.8-605113 & 0.78 & 0.88 & Scd \\
\hline ATHDFS_J223139.6-605039 & 0.25 & 0.19 & E/S0 \\
\hline ATHDFS_J223142.8-605024 & 0.51 & 0.33 & E/S0 \\
\hline ATHDFS_J223238.5-605018 & 0.57 & 0.59 & E/S0 \\
\hline ATHDFS_J223411.6-604931 & 0.02 & 0.24 & Sbc \\
\hline ATHDFS_J223221.3-604929 & 0.51 & 0.52 & E/S0 \\
\hline ATHDFS_J223223.6-604923 & 0.34 & 0.52 & Sbc \\
\hline ATHDFS_J223301.3-604926 & 0.42 & 0.63 & Sbc \\
\hline ATHDFS_J223414.3-604923 & 0.42 & 0.32 & E/S0 \\
\hline ATHDFS_J223524.8-604918A & 0.78 & 0.74 & Sbc \\
\hline ATHDFS_J223524.8-604918B & $\ldots$ & 0.92 & Sbc \\
\hline ATHDFS_J223413.1-604909 & 0.19 & 0.31 & Scd \\
\hline ATHDFS_J223107.4-604855 & $\ldots$ & 0.31 & E/S0 \\
\hline ATHDFS_J223400.5-604903B & $\ldots$ & 0.77 & Irr \\
\hline ATHDFS_J223359.4-604901 & & 0.59 & Irr \\
\hline ATHDFS_J223543.9-604838 & 0.45 & 0.37 & E/S0 \\
\hline ATHDFS_J223235.6-604844 & ... & 0.61 & $\mathrm{E} / \mathrm{S} 0$ \\
\hline ATHDFS_J223452.0-604834 & 0.48 & 0.31 & Sbc \\
\hline ATHDFS_J223337.8-604833 & 0.67 & 0.53 & Sbc \\
\hline ATHDFS_J223255.7-604823 & 0.78 & 0.77 & Sbc \\
\hline ATHDFS_J223250.5-604814 & 0.74 & 0.59 & Scd \\
\hline ATHDFS_J223243.0-604758 & 0.60 & 0.71 & Scd \\
\hline ATHDFS_J223414.7-604753 & 0.03 & 0.26 & Sbc \\
\hline ATHDFS_J223217.6-604751 & 1.24 & 0.22 & SB1 \\
\hline ATHDFS_J223417.5-604749 & 0.04 & 0.14 & Scd \\
\hline ATHDFS_J223226.8-604745 & 0.71 & 0.64 & Sbc \\
\hline ATHDFS_J223353.3-604723 & 2.05 & ... & \\
\hline ATHDFS_J223444.0-604710 & $\ldots$ & $0 .-$ & SB1 \\
\hline ATHDFS_J223315.8-604707 & $\ldots$ & 0.55 & E/S0 \\
\hline ATHDFS_J223527.8-604639A & $\ldots$ & 0.31 & E/S0 \\
\hline ATHDFS_J223231.6-604654 & $\ldots$ & 0.92 & Sbc \\
\hline ATHDFS_J223254.0-604650 & $\ldots$ & 0.59 & $\mathrm{Sbc}$ \\
\hline ATHDFS_J223305.3-604647 & 0.34 & 0.26 & E/S0 \\
\hline ATHDFS_J223118.9-604644 & 1.15 & 0.86 & Scd \\
\hline ATHDFS_J223333.2-604642 & 0.23 & 0.15 & E/S0 \\
\hline ATHDFS_J223228.5-604642 & 0.74 & 0.74 & Sbc \\
\hline ATHDFS_J223536.8-604632 & 0.03 & 0.25 & $\mathrm{Sbc}$ \\
\hline ATHDFS_J223330.4-604624 & 0.45 & 0.34 & E/S0 \\
\hline ATHDFS_J223310.2-604601 & $\ldots$ & 0.47 & Sbc \\
\hline ATHDFS_J223342.9-604524 & 0.16 & 0.36 & $\mathrm{Sbc}$ \\
\hline ATHDFS_J223324.0-604516 & 0.42 & 0.32 & $\mathrm{E} / \mathrm{SO}$ \\
\hline ATHDFS_J223320.1-604457 & & 0.31 & Scd \\
\hline ATHDFS_J223443.3-604452 & 1.70 & 0.10 & SB1 \\
\hline ATHDFS_J223311.5-604449 & 1.06 & 0.51 & SB2 \\
\hline ATHDFS_J223319.1-604428 & 1.24 & 0.74 & SB2 \\
\hline ATHDFS_J223210.3-604433 & 0.71 & 0.69 & E/S0 \\
\hline ATHDFS_J223439.0-604435 & 0.48 & 0.66 & $\mathrm{Sbc}$ \\
\hline ATHDFS_J223244.1-604437 & 0.32 & 0.44 & SB1 \\
\hline
\end{tabular}


Table 11

(Continued)

\begin{tabular}{|c|c|c|c|}
\hline ATHDF-S name & Teplitz et al. (2001) redshift & Redshift (this work) & Spectral type \\
\hline ATHDFS_J223147.2-604415 & 0.48 & 0.44 & E/S0 \\
\hline ATHDFS_J223326.3-604416 & $\ldots$ & 0.17 & E/S0 \\
\hline ATHDFS_J223422.4-604412 & 0.89 & $\ldots$ & $\ldots$ \\
\hline ATHDFS_J223226.8-604408 & 0.86 & 0.86 & $\mathrm{Sbc}$ \\
\hline ATHDFS_J223313.9-604359 & 1.99 & 0.23 & Scd \\
\hline ATHDFS_J223101.3-604351 & 1.49 & 0.04 & Sbc \\
\hline ATHDFS_J223147.4-604338 & 0.60 & 0.29 & SB2 \\
\hline ATHDFS_J223126.2-604337 & 0.01 & $\ldots$ & $\ldots$ \\
\hline ATHDFS_J223534.3-604328 & 0.64 & 0.53 & SB1 \\
\hline ATHDFS_J223250.6-604336 & 1.11 & $\ldots$ & $\ldots$ \\
\hline ATHDFS_J223522.2-604326 & 1.87 & 0.21 & Scd \\
\hline ATHDFS_J223440.4-604309 & 0.34 & 0.35 & Scd \\
\hline ATHDFS_J223430.8-604310 & 0.34 & 0.35 & Scd \\
\hline ATHDFS_J223240.2-604306 & 0.51 & 0.33 & Scd \\
\hline ATHDFS_J223207.9-604305 & 0.74 & 0.53 & Scd \\
\hline ATHDFS_J223130.4-604232 & $\ldots$ & 0.67 & E/S0 \\
\hline ATHDFS_J223239.0-604230 & 0.03 & 0.05 & $\mathrm{E} / \mathrm{S} 0$ \\
\hline ATHDFS_J223312.4-604227 & 0.57 & 0.41 & $\mathrm{Sbc}$ \\
\hline ATHDFS_J223437.2-604214 & 0.45 & 0.37 & E/S0 \\
\hline ATHDFS_J223338.8-604216 & 1.65 & 0.32 & Irr \\
\hline ATHDFS_J223523.6-604207 & 0.48 & 0.39 & E/S0 \\
\hline ATHDFS_J223135.8-604207 & 0.40 & 0.32 & E/S0 \\
\hline ATHDFS_J223359.8-604155 & 0.10 & 0.08 & Sbc \\
\hline ATHDFS_J223255.4-604151 & 0.16 & 0.18 & SB2 \\
\hline ATHDFS_J223158.4-604142 & 0.82 & 0.79 & Sbc \\
\hline ATHDFS_J223339.5-604131 & $\ldots$ & 0.33 & $\mathrm{Sbc}$ \\
\hline ATHDFS_J223224.5-604113 & 0.60 & 0.64 & E/S0 \\
\hline ATHDFS_J223448.3-604042 & 0.11 & 0.21 & Scd \\
\hline ATHDFS_J223430.1-604043 & 0.60 & 0.51 & Sbc \\
\hline ATHDFS_J223337.5-604006 & 0.40 & 0.34 & Scd \\
\hline ATHDFS_J223438.0-603951 & 0.42 & 0.38 & E/S0 \\
\hline ATHDFS_J223047.9-603933 & 0.57 & 0.44 & Sbc \\
\hline ATHDFS_J223220.6-603931 & 0.30 & 0.23 & E/S0 \\
\hline ATHDFS_J223529.2-603927 & 0.54 & 0.58 & E/S0 \\
\hline ATHDFS_J223400.2-603930 & 0.71 & 0.68 & Scd \\
\hline ATHDFS_J223222.7-603924 & 0.54 & 0.43 & Scd \\
\hline ATHDFS_J223253.7-603921 & 0.54 & 0.73 & Sbc \\
\hline ATHDFS_J223331.3-603914 & 0.48 & 0.38 & E/S0 \\
\hline ATHDFS_J223123.1-603903 & 0.16 & 0.06 & Sbc \\
\hline ATHDFS_J223245.6-603857 & $\ldots$ & 0.75 & Irr \\
\hline ATHDFS_J223144.4-603858 & 0.32 & 0.26 & E/S0 \\
\hline ATHDFS_J223153.7-603853 & 0.89 & 0.52 & Irr \\
\hline ATHDFS_J223450.4-603844 & 0.42 & 0.34 & E/S0 \\
\hline ATHDFS_J223307.1-603846 & 0.64 & 0.55 & Sbc \\
\hline ATHDFS_J223457.3-603840 & 0.64 & 0.52 & $\mathrm{Sbc}$ \\
\hline ATHDFS_J223232.3-603842 & $\ldots$ & 0.23 & E/SO \\
\hline ATHDFS_J223304.6-603835 & 0.71 & 0.59 & $\mathrm{Sbc}$ \\
\hline ATHDFS_J223138.7-603818 & $\ldots$ & 0.62 & $\mathrm{Sbc}$ \\
\hline ATHDFS_J223248.2-603805 & 0.54 & 0.40 & Sbc \\
\hline ATHDFS_J223334.4-603804 & 0.54 & 0.56 & Irr \\
\hline ATHDFS_J223436.9-603754 & 1.29 & 0.97 & $\mathrm{Sbc}$ \\
\hline ATHDFS_J223254.5-603748 & 0.21 & 0.23 & Sbc \\
\hline ATHDFS_J223350.0-603741 & $\ldots$ & 0.61 & E/S0 \\
\hline ATHDFS_J223232.8-603737 & $\ldots$ & 0.77 & E/S0 \\
\hline ATHDFS_J223404.8-603732 & $\ldots$ & 0.98 & $\mathrm{Sbc}$ \\
\hline ATHDFS_J223437.7-603726 & 0.48 & 0.37 & Scd \\
\hline ATHDFS_J223417.1-603724 & 0.07 & 0.32 & $\mathrm{Sbc}$ \\
\hline ATHDFS_J223341.2-603724 & 0.82 & 0.88 & Scd \\
\hline ATHDFS_J223225.6-603717 & 0.54 & 0.45 & Scd \\
\hline ATHDFS_J223506.5-603700 & 0.34 & 0.05 & SB2 \\
\hline ATHDFS_J223400.2-603653 & 0.12 & 0.08 & Sbc \\
\hline ATHDFS_J223525.8-603652 & 0.86 & 0.88 & $\mathrm{Sbc}$ \\
\hline ATHDFS_J223404.3-603638 & 0.48 & 0.42 & E/S0 \\
\hline ATHDFS_J223406.7-603637 & 0.01 & 0.10 & Scd \\
\hline ATHDFS_J223341.9-603634 & $\ldots$ & 0.04 & Scd \\
\hline ATHDFS_J223451.7-603632 & 0.32 & 0.29 & $\mathrm{Sbc}$ \\
\hline
\end{tabular}


Table 11

(Continued)

\begin{tabular}{|c|c|c|c|}
\hline ATHDF-S name & Teplitz et al. (2001) redshift & Redshift (this work) & Spectral type \\
\hline ATHDFS_J223316.5-603627 & 0.60 & 0.29 & SB2 \\
\hline ATHDFS_J223526.2-603617 & 0.51 & 0.37 & $\mathrm{Sbc}$ \\
\hline ATHDFS_J223518.7-603619 & & 0.12 & SB1 \\
\hline ATHDFS_J223158.5-603614 & 0.51 & 0.42 & Scd \\
\hline ATHDFS_J223421.8-603603 & 2.05 & 0.22 & Scd \\
\hline ATHDFS_J223232.4-603542A & 0.42 & $\ldots$ & $\ldots$ \\
\hline ATHDFS_J223232.4-603542B & 0.42 & $\ldots$ & $\ldots$ \\
\hline ATHDFS_J223104.8-603549 & 0.71 & 0.58 & Scd \\
\hline ATHDFS_J223525.7-603544 & 0.04 & 0.06 & Scd \\
\hline ATHDFS_J223224.0-603537 & & 0.17 & Scd \\
\hline ATHDFS_J223253.0-603539 & 0.74 & 0.58 & Scd \\
\hline ATHDFS_J223245.3-603537 & & 0.51 & Scd \\
\hline ATHDFS_J223046.1-603525 & 0.57 & 0.44 & Sbc \\
\hline ATHDFS_J223338.8-603523 & 0.16 & 0.14 & E/S0 \\
\hline ATHDFS_J223208.3-603519 & 0.45 & 0.36 & Sbc \\
\hline ATHDFS_J223344.9-603515 & 0.51 & 0.46 & Scd \\
\hline ATHDFS_J223524.8-603509 & & 0.58 & Sbc \\
\hline ATHDFS_J223536.3-603506 & 0.89 & 0.96 & $\mathrm{Sbc}$ \\
\hline ATHDFS_J223549.5-603502 & 0.57 & 0.50 & $\mathrm{Sbc}$ \\
\hline ATHDFS_J223350.5-603503 & & 0.98 & E/S0 \\
\hline ATHDFS_J223541.5-603454 & 0.64 & 0.53 & Sbc \\
\hline ATHDFS_J223438.6-603450 & 0.30 & 0.19 & E/S0 \\
\hline ATHDFS_J223103.2-603453 & 0.57 & 0.58 & E/S0 \\
\hline ATHDFS_J223425.0-603452 & & 0.72 & SB2 \\
\hline ATHDFS_J223212.3-603448 & 0.08 & 0.17 & Scd \\
\hline ATHDFS_J223207.4-603445 & 0.37 & 0.30 & $\mathrm{E} / \mathrm{S} 0$ \\
\hline ATHDFS_J223243.3-603443 & 0.57 & 0.46 & Sbc \\
\hline ATHDFS_J223442.8-603433 & 0.57 & 0.30 & Scd \\
\hline ATHDFS_J223216.6-603434 & 1.15 & 0.83 & Scd \\
\hline ATHDFS_J223539.3-603424 & . & 0.87 & Sbc \\
\hline ATHDFS_J223153.2-603422 & 0.42 & 0.35 & E/S0 \\
\hline ATHDFS_J223401.0-603424 & & 0.13 & E/S0 \\
\hline ATHDFS_J223245.5-603419 & 0.54 & 0.39 & $\mathrm{Sbc}$ \\
\hline ATHDFS_J223311.9-603417 & 0.42 & 0.64 & $\mathrm{Sbc}$ \\
\hline ATHDFS_J223434.1-603410 & & 0.76 & Irr \\
\hline ATHDFS_J223053.2-603402 & 1.15 & 0.94 & Scd \\
\hline ATHDFS_J223415.0-603408 & 0.57 & 0.48 & Scd \\
\hline ATHDFS_J223409.7-603402 & 0.78 & 0.79 & Scd \\
\hline ATHDFS_J223512.7-603353 & 0.57 & 0.58 & E/S0 \\
\hline ATHDFS_J223306.0-603350 & 0.17 & 0.30 & Scd \\
\hline ATHDFS_J223329.7-603352 & & 0.11 & SB1 \\
\hline ATHDFS_J223243.4-603352 & 1.24 & 0.81 & SB1 \\
\hline ATHDFS_J223138.5-603344 & $\ldots$ & 0.24 & E/S0 \\
\hline ATHDFS_J223420.9-603336 & 1.11 & 0.10 & SB1 \\
\hline ATHDFS_J223225.0-603338 & 0.27 & 0.35 & Scd \\
\hline ATHDFS_J223513.7-603333 & 0.51 & 0.36 & $\mathrm{Sbc}$ \\
\hline ATHDFS_J223247.6-603337 & 0.67 & 0.56 & $\mathrm{Sbc}$ \\
\hline ATHDFS_J223337.5-603329 & 1.34 & $\ldots$ & $\ldots$ \\
\hline ATHDFS_J223253.1-603329 & 1.15 & 0.60 & Scd \\
\hline ATHDFS_J223121.6-603301 & 1.02 & $\ldots$ & $\ldots$ \\
\hline ATHDFS_J223308.6-603251 & 0.64 & 0.64 & E/S0 \\
\hline ATHDFS_J223456.8-603251 & $\ldots$ & 0.20 & SB1 \\
\hline ATHDFS_J223229.5-603243 & & 0.75 & Irr \\
\hline ATHDFS_J223509.4-603235 & 3.06 & 0.22 & Sbc \\
\hline ATHDFS_J223142.5-603238 & $\ldots$ & 0.42 & $\mathrm{Sbc}$ \\
\hline ATHDFS_J223317.7-603235 & $\ldots$ & 0.60 & E/S0 \\
\hline ATHDFS_J223429.9-603226 & 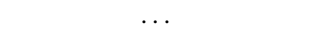 & 0.21 & E/S0 \\
\hline ATHDFS_J223331.6-603222 & 0.57 & 0.42 & Scd \\
\hline ATHDFS_J223504.4-603221 & 0.54 & 0.45 & $\mathrm{Sbc}$ \\
\hline ATHDFS_J223343.5-603211 & 0.60 & 0.53 & Sbc \\
\hline ATHDFS_J223519.4-603157A & 0.51 & 0.38 & Sbc \\
\hline ATHDFS_J223519.4-603157B & 0.51 & 0.38 & Scd \\
\hline ATHDFS_J223433.9-603150 & & 0.75 & Irr \\
\hline ATHDFS_J223449.7-603137 & 0.67 & 0.83 & Sbc \\
\hline ATHDFS_J223316.0-603127 & 0.60 & 0.52 & Sbc \\
\hline ATHDFS_J223548.9-603113 & 0.51 & 0.39 & E/S0 \\
\hline
\end{tabular}


Table 11

(Continued)

\begin{tabular}{|c|c|c|c|}
\hline ATHDF-S name & Teplitz et al. (2001) redshift & Redshift (this work) & Spectral type \\
\hline ATHDFS_J223201.4-603118 & 0.60 & 0.59 & E/S0 \\
\hline ATHDFS_J223351.7-603117 & 0.67 & 0.49 & Scd \\
\hline ATHDFS_J223504.6-603109 & 0.08 & 0.05 & $\mathrm{Sbc}$ \\
\hline ATHDFS_J223415.5-603108 & 1.20 & $\ldots$ & $\ldots$ \\
\hline ATHDFS_J223447.4-603050 & 0.89 & $\ldots$ & $\ldots$ \\
\hline ATHDFS_J223404.0-603037A & 1.29 & $\ldots$ & $\ldots$ \\
\hline ATHDFS_J223404.0-603037B & 0.48 & 0.17 & E/S0 \\
\hline ATHDFS_J223445.6-603032 & 1.34 & 0.98 & Scd \\
\hline ATHDFS_J223304.8-603031 & 0.54 & 0.40 & Scd \\
\hline ATHDFS_J223120.1-603025 & 0.51 & 0.37 & $\mathrm{Sbc}$ \\
\hline ATHDFS_J223241.4-603025 & 0.54 & 0.41 & Scd \\
\hline ATHDFS_J223440.4-603017 & 0.27 & 0.19 & E/S0 \\
\hline ATHDFS_J223216.6-603016 & 0.48 & 0.33 & Sbc \\
\hline ATHDFS_J223453.7-603008 & 0.45 & 0.35 & E/S0 \\
\hline ATHDFS_J223430.1-602959 & 0.01 & 0.28 & Irr \\
\hline ATHDFS_J223224.7-603005 & 1.44 & .. & $\cdots$ \\
\hline ATHDFS_J223058.4-602952 & 0.82 & 0.82 & $\mathrm{Sbc}$ \\
\hline ATHDFS_J223530.9-602951 & 1.20 & & . \\
\hline ATHDFS_J223534.2-602948 & 0.23 & 0.21 & $\mathrm{Sbc}$ \\
\hline ATHDFS_J223108.1-602946 & & 0.32 & E/S0 \\
\hline ATHDFS_J223316.8-602934 & 0.89 & 0.12 & Scd \\
\hline ATHDFS_J223513.7-602930 & 1.44 & 0.08 & Sbc \\
\hline ATHDFS_J223454.1-602926 & 0.57 & 0.56 & E/S0 \\
\hline ATHDFS_J223415.3-602925 & 0.21 & 0.21 & $\mathrm{Sbc}$ \\
\hline ATHDFS_J223149.1-602924 & 1.24 & 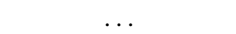 & $\ldots$ \\
\hline ATHDFS_J223157.5-602919 & 0.67 & 0.36 & $\mathrm{Sbc}$ \\
\hline ATHDFS_J223447.1-602915 & 0.42 & 0.31 & E/S0 \\
\hline ATHDFS_J223317.7-602916 & 1.54 & 0.04 & Irr \\
\hline ATHDFS_J223515.2-602856 & 0.60 & 0.47 & Sbc \\
\hline ATHDFS_J223420.1-602901 & .. & 0.87 & Sbc \\
\hline ATHDFS_J223445.3-602855 & 1.54 & & \\
\hline ATHDFS_J223431.7-602859 & 0.51 & 0.39 & Scd \\
\hline ATHDFS_J223212.1-602858 & 0.57 & 0.42 & $\mathrm{Sbc}$ \\
\hline ATHDFS_J223505.0-602853 & 0.48 & 0.45 & $\mathrm{E} / \mathrm{S} 0$ \\
\hline ATHDFS_J223236.2-602855 & 0.34 & 0.40 & SB2 \\
\hline ATHDFS_J223307.7-602853 & 1.44 & 0.54 & Scd \\
\hline ATHDFS_J223326.9-602850 & 0.02 & 0.02 & Scd \\
\hline ATHDFS_J223330.5-602849 & 0.21 & 0.35 & SB2 \\
\hline ATHDFS_J223138.5-602834 & $\ldots$ & 0.76 & Irr \\
\hline ATHDFS_J223307.0-602827 & $\ldots$ & 0.07 & SB2 \\
\hline ATHDFS_J223436.2-602821 & $\ldots$ & 0.89 & Scd \\
\hline ATHDFS_J223329.4-602811 & $\ldots$ & 0.24 & SB1 \\
\hline ATHDFS_J223413.3-602808 & $\ldots$ & 0.66 & Scd \\
\hline ATHDFS_J223114.1-602800 & $\ldots$ & 0.75 & Irr \\
\hline ATHDFS_J223133.4-602755 & 0.60 & 0.46 & $\mathrm{Sbc}$ \\
\hline ATHDFS_J223443.9-602739A & 0.37 & 0.31 & $\mathrm{E} / \mathrm{SO}$ \\
\hline ATHDFS_J223443.9-602739B & 0.37 & 0.31 & $\mathrm{E} / \mathrm{SO}$ \\
\hline ATHDFS_J223443.9-602739C & 0.37 & 0.31 & E/S0 \\
\hline ATHDFS_J223136.1-602731 & 0.48 & 0.35 & $\mathrm{Sbc}$ \\
\hline ATHDFS_J223142.7-602719 & 0.60 & 0.47 & Sbc \\
\hline ATHDFS_J223332.7-602723 & 0.64 & 0.71 & Scd \\
\hline ATHDFS_J223227.6-602719A & 0.98 & 0.74 & SB2 \\
\hline ATHDFS_J223312.3-602707 & $\ldots$ & 0.86 & E/S0 \\
\hline ATHDFS_J223432.3-602652 & .. & 0.80 & $\mathrm{Sbc}$ \\
\hline ATHDFS_J223506.2-602647 & 0.64 & 0.46 & Scd \\
\hline ATHDFS_J223342.2-602639 & 0.51 & 0.60 & Irr \\
\hline ATHDFS_J223221.4-602629 & 1.20 & 0.70 & Scd \\
\hline ATHDFS_J223400.9-602633 & 0.37 & 0.31 & E/S0 \\
\hline ATHDFS_J223212.4-602632 & 0.78 & 0.73 & $\mathrm{Sbc}$ \\
\hline ATHDFS_J223136.2-602627 & 0.60 & 0.51 & Sbc \\
\hline ATHDFS_J223432.6-602614 & 1.15 & $\ldots$ & $\ldots$ \\
\hline ATHDFS_J223335.3-602615 & 0.08 & 0.09 & E/S0 \\
\hline ATHDFS_J223136.9-602610 & 0.60 & 0.49 & $\mathrm{Sbc}$ \\
\hline ATHDFS_J223442.5-602601 & 0.40 & 0.40 & Irr \\
\hline ATHDFS_J223148.3-602554 & 0.57 & 0.43 & Scd \\
\hline ATHDFS_J223357.8-602548 & 0.01 & 0.03 & Scd \\
\hline
\end{tabular}


Table 11

(Continued)

\begin{tabular}{lccc}
\hline \hline ATHDF-S name & Teplitz et al. (2001) redshift & Redshift (this work) & Spectral type \\
\hline ATHDFS_J223222.4-602532 & $\ldots$ & 0.46 & E/S0 \\
ATHDFS_J223351.2-602519 & 0.60 & 0.44 & Sbc \\
ATHDFS_J223141.1-602506 & 0.19 & 0.31 & Scd \\
ATHDFS_J223134.5-602457 & 0.37 & 0.56 & Sbc \\
ATHDFS_J223317.1-602427 & $\ldots$ & 0.07 & SB2 \\
ATHDFS_J223444.9-602417 & $\ldots$ & 0.08 & Scd \\
ATHDFS_J223436.8-602426 & 0.54 & 0.38 & Scd \\
ATHDFS_J223245.3-602407 & $\ldots$ & 0.49 & Sbc \\
ATHDFS_J223343.4-602348 & 0.14 & 0.20 & SB2 \\
ATHDFS_J223331.6-602307 & $\ldots$ & 0.59 & E/S0 \\
ATHDFS_J223358.9-602258 & 0.04 & $\ldots$ & $\ldots$ \\
ATHDFS_J223300.5-602225 & 0.51 & 0.43 & Scd \\
ATHDFS_J223154.6-602211 & 0.82 & 0.97 & Sbc \\
ATHDFS_J223353.6-602136 & $\ldots$ & 0.91 & Sbc \\
ATHDFS_J223341.1-602054 & 1.02 & $\ldots$ & $\ldots$ \\
ATHDFS_J223431.4-602041 & $\ldots$. & 0.31 & E/S0 \\
ATHDFS_J223356.6-601949 & 0.27 & 0.21 & E/S0 \\
ATHDFS_J223316.0-601939 & 0.45 & 0.42 & E/S0 \\
\hline
\end{tabular}

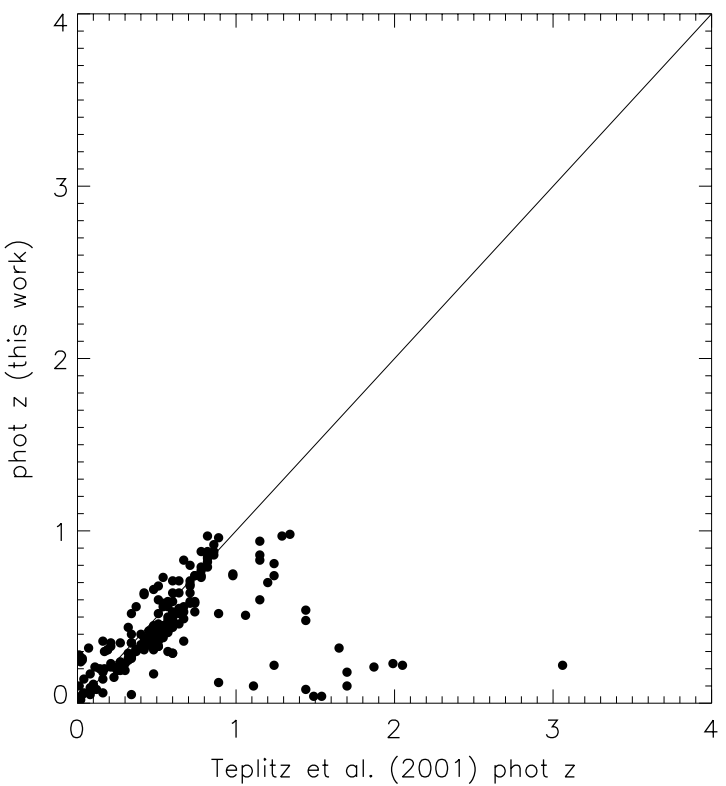

Figure 15. Comparison of Teplitz et al. (2001) and our photometric redshifts for the 209 ATHDF-S sources that have both

radio sources $\left(S_{1.4 \mathrm{GHz}}<0.1 \mathrm{mJy}\right)$. This trend of fainter radio sources having fainter optical hosts was also observed by Afonso et al. (2006) (but see Barger et al. 2007).

We plot the absolute $I$ magnitude versus redshift in Figure 18 for the 266 radio sources with redshift information. The magnitudes are $k$-corrected using the best-fit spectral type from the photometric redshift analysis. For six radio sources there was a $2 \mathrm{dF}$ spectroscopic redshift but no photometric type. The $k$-correction in these cases was determined from a starburst template for the $2 \mathrm{dF}$ emission line galaxies (two total) and an $\mathrm{E} / \mathrm{S} 0$ template for the $2 \mathrm{dF}$ absorption line galaxies (two total). A further two cases were high redshift $(z>2) 2 \mathrm{dF}$ broadline emitting quasars, which we $k$-corrected with a QSO composite template from Francis et al. (1991). The approximate $I$ magnitude limit of 23.5 is shown by the solid line in Figure 18. The host galaxies span a small range in absolute magnitude across the redshift range $0<z<1$. We find that the median absolute
$I$ magnitude for $z<0.5$ sources is -21.7 , while the median absolute $I$ magnitude for sources at $0.5<z<1.0$ is -22.3 . So there may be a trend toward these radio sources having brighter host galaxies at $0.5<z<1.0$, but this trend is very weak.

We show absolute $I$ magnitude versus $1.4 \mathrm{GHz}$ radio power for all radio sources with redshift information in Figure 19. The majority of the radio sources $(\sim 80 \%)$ lie in the magnitude range -20 to -24 . This corresponds to 1.6 dex in luminosity. In comparison, the radio powers have a wider range of luminosities (about 2 dex encompasses $80 \%$ of the sources). The higher redshift sources must have higher radio powers, but the median absolute $I$ magnitude does not change noticeably with redshift (Figure 18). So the optical properties of the host galaxies are not changing as fast as the radio power of the host galaxies (see Barger et al. 2007).

In Figure 20 we plot the restframe $B-I$ colors of the optically identified radio sources in our sample against $1.4 \mathrm{GHz}$ flux density and redshift. The solid, dotted, and dashed lines in Figure 20 show the restframe colors of a Coleman et al. (1980) E/S0, Sbc, and Irr galaxy, respectively. The host galaxies have colors that span the range between E/S0 to Irr, but have a median color that is Sbc like. We find little variation in the median color of the host galaxies with either flux density or redshift. Smolčić et al. (2008) also find no change in the rest frame colors of sub-mJy radio sources out to redshift $z=1.3$. This supports the suggestion that the host galaxies of faint radio sources are drawn from the same population as that of bright radio sources (see Barger et al. 2007).

\subsection{Radio-to-Optical Ratios}

The radio-to-optical ratio of a radio source can give clues to the nature of the radio emission and it has traditionally been used to determine the "radio loudness" of radio sources (Kellermann et al. 1989). While optically selected samples of quasars have been found to have a wide range of radio flux densities, and thus radio-to-optical ratios (Sramek \& Weedman 1980; Condon et al. 1981), it has been suggested that the distribution of this ratio is bimodal (e.g., Kellermann et al. 1989; Stocke et al. 1992). These authors find a dip in the distribution of $5 \mathrm{GHz}$ to $B$-band flux density ratios at $3<r=S_{5 \mathrm{GHz}} / S_{B-\text { band }}<30$, and formally classify radio sources as radio loud if they have $r>10$. 

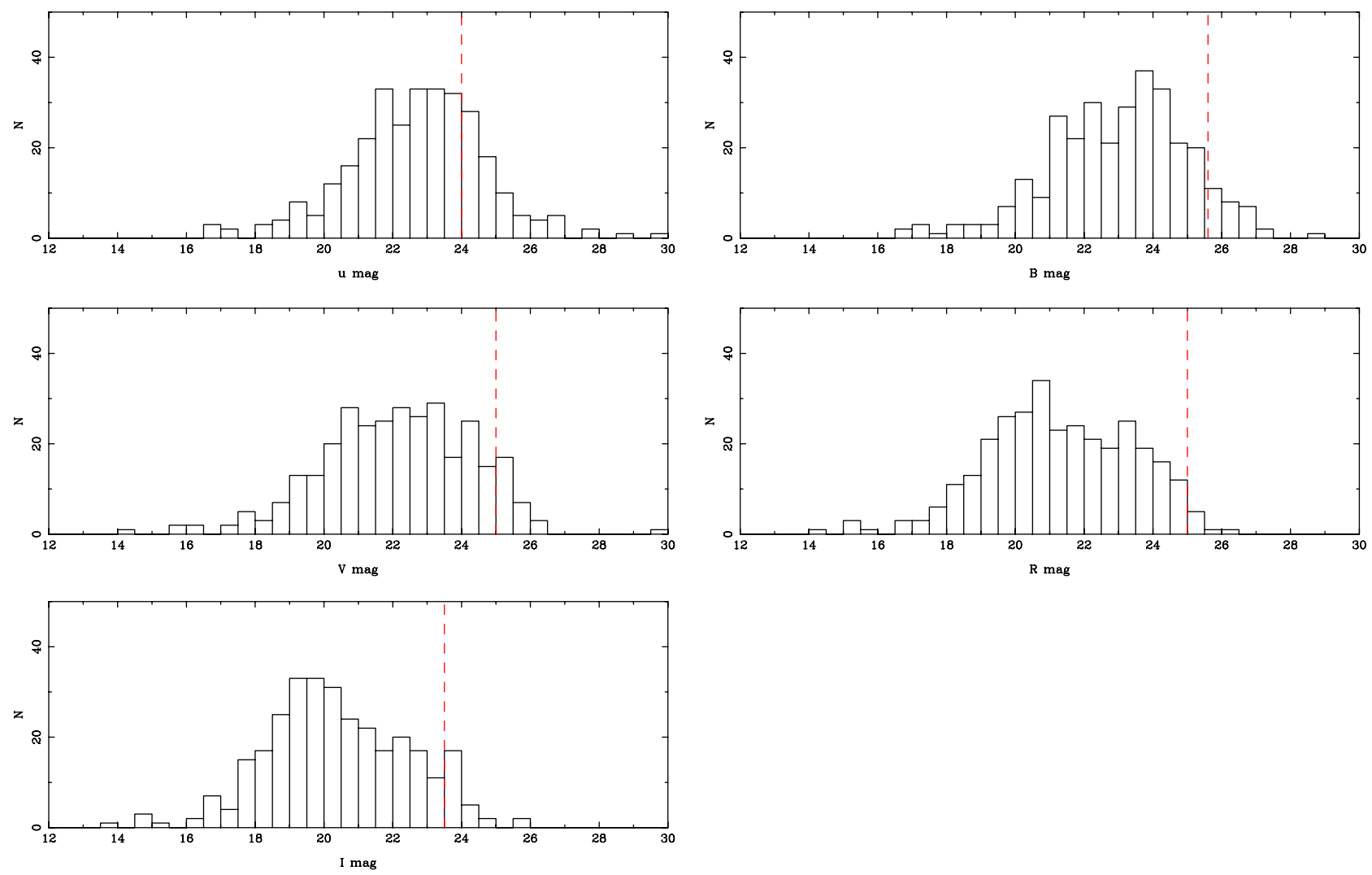

Figure 16. $u B V R I$ magnitude distribution of the 315 ATHDF-S sources with CTIO counterparts.

(A color version of this figure is available in the online journal)
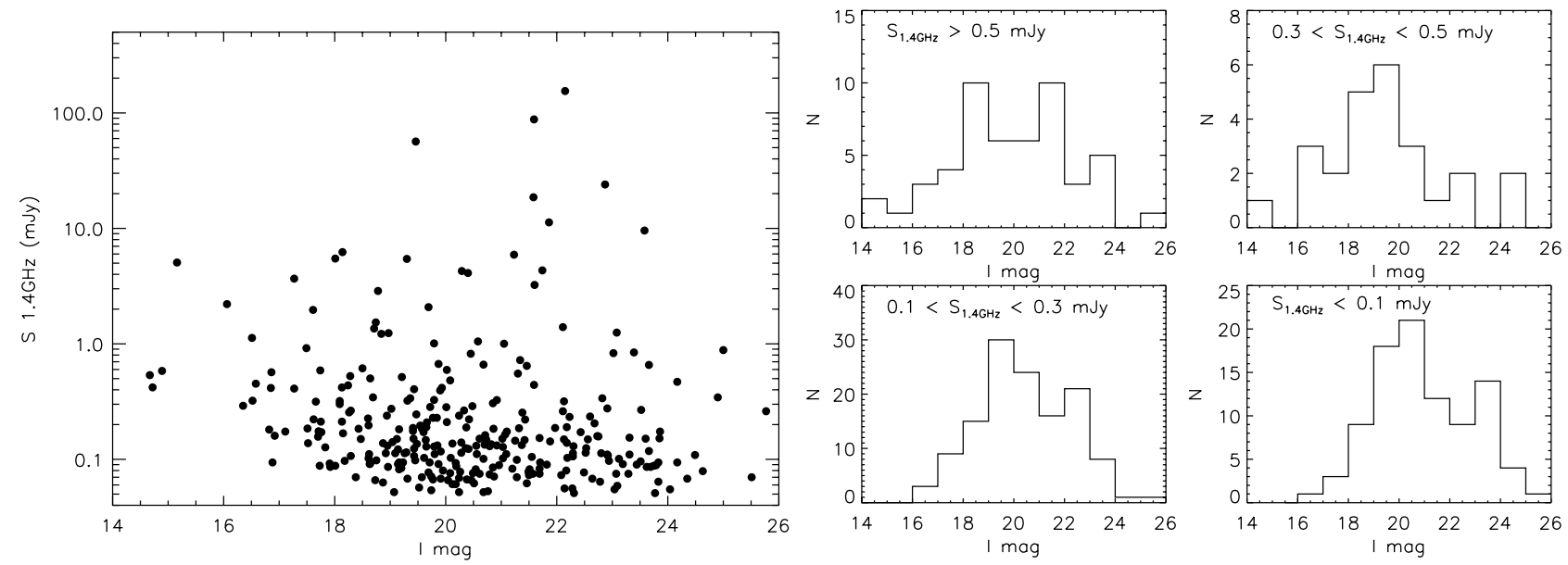

Figure 17. Left panel: the $1.4 \mathrm{GHz}$ flux density versus $I$ magnitude for the radio sources detected in the CTIO $I$-band image. Right panel: the $I$ magnitude distributions for the radio sources for flux density bins as shown.

The bimodality of radio loudness is still controversial, though, with White et al. (2000) claiming no bimodality is seen for a radio-selected sample of optically bright quasars from the FIRST Bright Quasar Survey. However, Ivezić et al. (2002) combined the FIRST survey with deeper SDSS data and found evidence for a bimodality with a minimum in the radio-to-optical ratio between 1 and 10. Ivezić et al. (2002) claim that the FIRST Bright Quasar Survey was biased to radio-intermediate sources with ratios between 1 and 10 because of the brighter $I \lesssim 18$ limit and hence no bimodality was observed. Moreover, a study of the nuclear region of local Seyferts has found that $60 \%$ of them meet the radio-loud $r>10$ criterion, suggesting that, after removing the effects of the diluting host galaxy, most Seyferts are not radio quiet (Ho \& Peng 2001).

We plot the observed radio-to- $I$-band flux density ratio against redshift in Figure 21. The $I$-band was selected as it is the reddest filter and hence covers the restframe optical regime to the highest redshift. Marked in Figure 21 are ratios from the radio-loud and radio-quiet QSOs from Elvis et al. (1994). We also overplot tracks from local starbursts Arp220 and M82, which have star-formation rates of several hundred and tens of $M_{\odot} \mathrm{yr}^{-1}$, respectively. The radio-to-optical ratio tracks for 


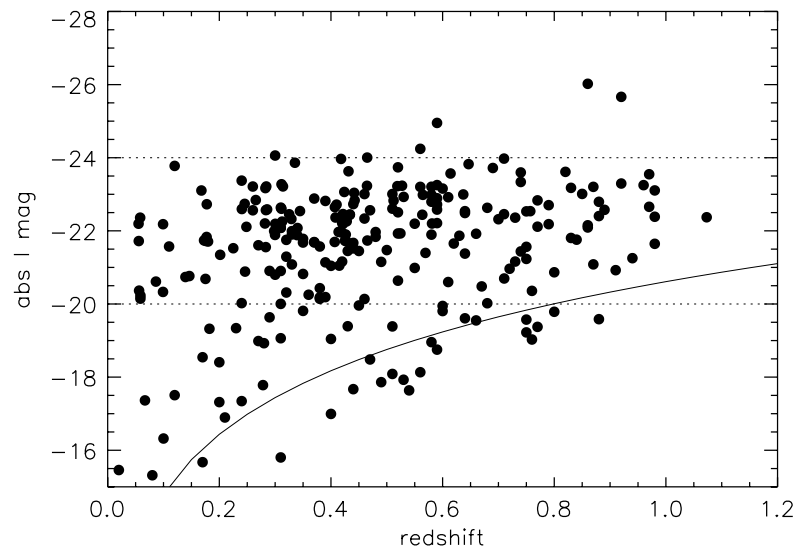

Figure 18. Absolute $I$-band magnitude versus redshift for the 266 radio sources with redshift information. The solid line approximates the limit of the optical survey $(I=23.5)$. The dotted lines show the range in magnitude which encompasses about $80 \%$ of the radio sources.

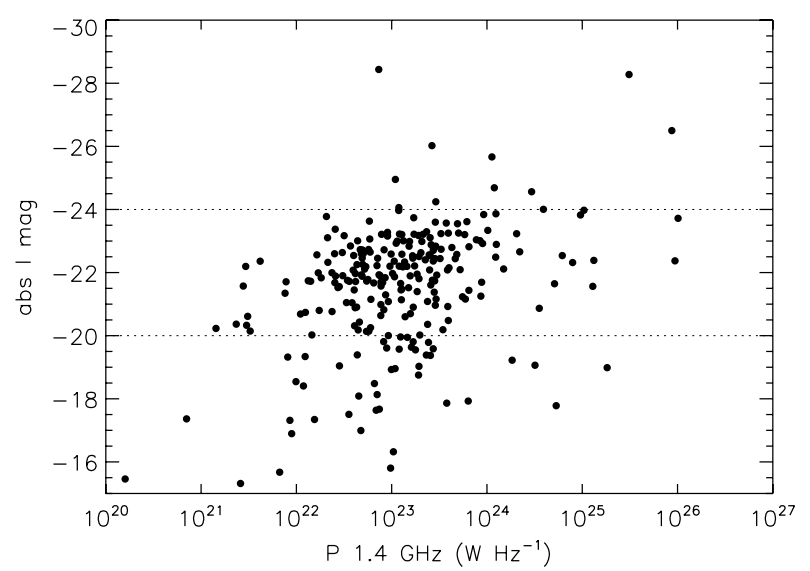

Figure 19. Absolute $I$-band magnitude versus radio power, $P 1.4 \mathrm{GHz}$, for the 266 radio sources with redshift information. The dotted lines show the range in magnitude which encompasses about $80 \%$ of the radio sources.

the starbursts cover the intermediate range of $1-10$. We find that the majority of the optically detected radio sources have radio-to-optical ratios in the range $1-100$, with a median of $S_{1.4 \mathrm{GHz}} / S_{I-\text { band }}=10.6$.

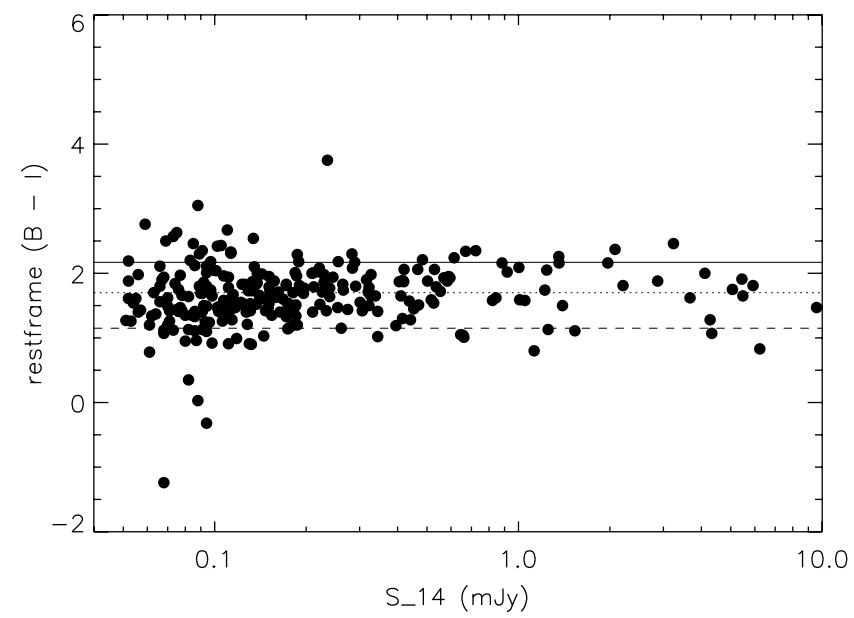

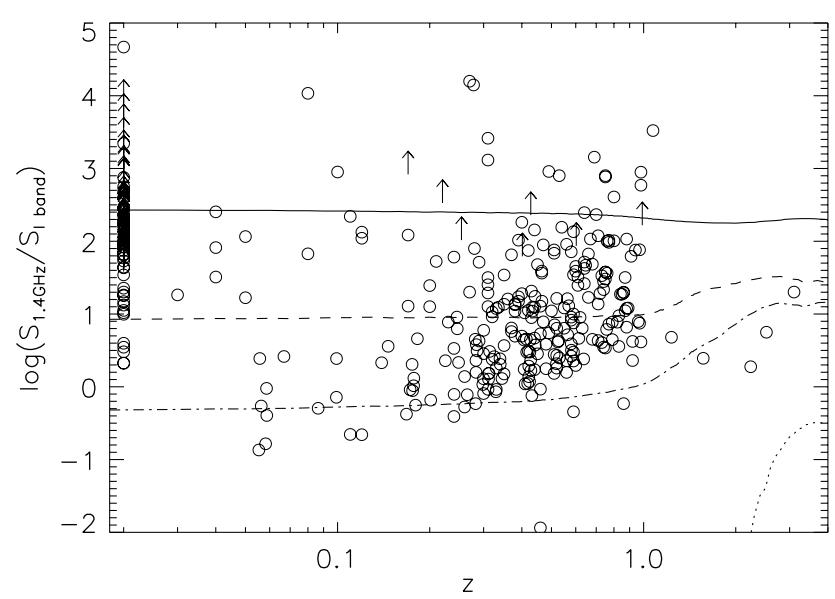

Figure 21. Radio-to-optical flux density ratio for sources in the ATHDF-S, plotted against redshift. The arrows mark lower limits of ATHDF-S sources not detected in the $I$-band imaging. Sources without a redshift are plotted at $z=0.02$. The tracks for a radio loud (black solid line) and radio quiet (dotted line) from Elvis et al. (1994) are plotted for comparison. Also plotted are the ratios from prototypical starbursts Arp220 (dashed line) and M82 (dot-dashed line).

Care must be taken when interpreting the radio-to-optical ratio. At high redshifts we observe objects at a different cosmological epoch, so they may have broadband spectral energy distribution (SED) properties which differ from local objects. For instance, there is evidence that high redshift starbursts and ultraluminous infrared galaxies (ULIRGs) have SEDs that differ from local analogs (Pope et al. 2006; Huynh et al. 2007b). Also, sources at earlier epochs may be more dust obscured, which would increase the radio-to-optical ratio. The tracks in Figure 21 are therefore a rough guide only. A further complicating factor is that AGNs have variable luminosities. The luminosity of an AGN can change on timescales of minutes (X-ray) to months (radio/optical/IR) (e.g. Hawkins 2002). The effect of variability in the radio should be reduced since the radio observations were taken over a period of 3 years and any variability is therefore diluted. The optical data, however, was obtained over a period of only 3 days.

Because of these caveats we choose a conservative approach to using the radio-to-optical ratio as a radio-loudness parameter.

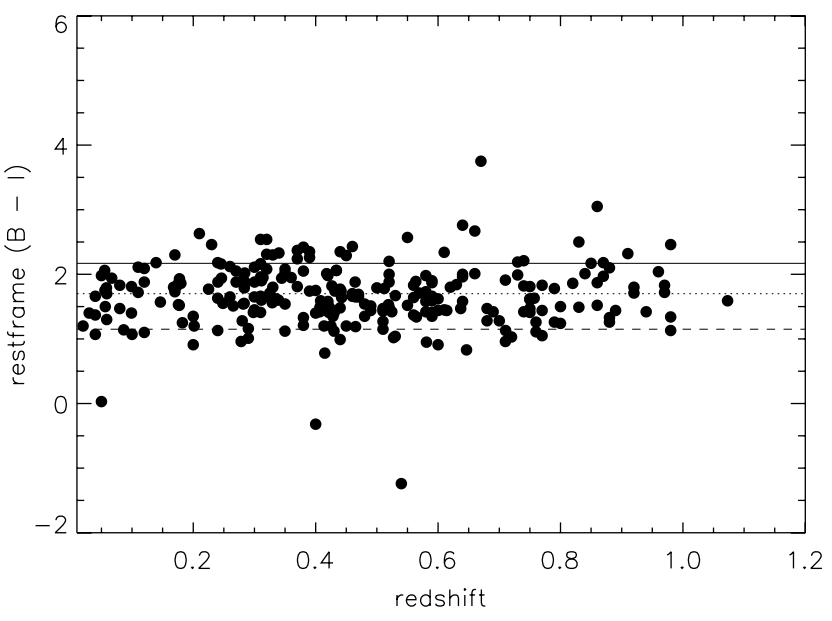

Figure 20. Left panel: the $1.4 \mathrm{GHz}$ flux density versus restframe $(B-I)$ color for the 266 radio sources with redshift information. The solid, dotted, and dashed lines show the restframe colors of an E/S0, Sbc and Irr galaxy (Coleman et al. 1980), respectively. Right panel: redshift versus restframe ( $B-I$ ) color for the 266 radio sources with redshift information. The lines are as for the left panel. 
Table 12

Summary of ATHDF-S Sources Deemed Radio Loud from the Radio-to-Optical Ratio

\begin{tabular}{|c|c|c|c|}
\hline ATHDF-S name & $S_{1.4 \mathrm{GHz}}$ & $I$ mag & $R_{I}$ \\
\hline ATHDF-S_J223353.9-605452 & 5.918 & 21.23 & 2.88 \\
\hline ATHDF-S_J223214.8-605430 & 18.601 & 21.58 & 3.52 \\
\hline ATHDF-S_J223448.4-605417 & 1.396 & 22.11 & 2.61 \\
\hline ATHDF-S_J223203.0-605242A & 87.888 & 21.59 & 4.20 \\
\hline ATHDF-S_J223107.4-604855 & 11.272 & 21.86 & 3.42 \\
\hline ATHDF-S_J223320.1-604457 & 0.344 & 24.90 & 3.12 \\
\hline ATHDF-S_J223210.3-604433 & 56.513 & 19.46 & 3.16 \\
\hline ATHDF-S_J223534.3-604328 & 0.657 & 23.66 & 2.90 \\
\hline ATHDF-S_J223355.6-604315 & 154.700 & 22.15 & 4.67 \\
\hline ATHDF-S_J223123.1-603903 & 23.988 & 22.87 & 4.15 \\
\hline ATHDF-S_J223245.6-603857 & 0.843 & 23.39 & 2.90 \\
\hline ATHDF-S_J223404.8-603732 & 3.238 & 21.60 & 2.77 \\
\hline ATHDF-S_J223343.7-603651 & 0.070 & 25.51 & 2.67 \\
\hline ATHDF-S_J223350.5-603503 & 1.252 & 23.08 & 2.95 \\
\hline ATHDF-S_J223420.9-603336 & 4.328 & 21.74 & 2.95 \\
\hline ATHDF-S_J223212.9-603234A & 2.816 & 22.02 & 2.88 \\
\hline ATHDF-S_J223212.9-603234B & 1.466 & 22.02 & 2.59 \\
\hline ATHDF-S_J223113.5-603147 & 0.831 & 23.02 & 2.75 \\
\hline ATHDF-S_J223329.1-602933 & 0.261 & 25.77 & 3.34 \\
\hline ATHDF-S_J223444.9-602417 & 9.581 & 23.58 & 4.03 \\
\hline ATHDF-S_J223245.3-602407 & 0.469 & 24.17 & 2.96 \\
\hline ATHDF-S_J223303.6-605751 & 0.508 & $>23.5$ & $>2.53$ \\
\hline ATHDF-S_J223410.5-605545A & 2.106 & $>23.5$ & $>3.14$ \\
\hline ATHDF-S_J223410.5-605545B & 2.443 & $>23.5$ & $>3.21$ \\
\hline ATHDF-S_J223308.5-605544 & 3.643 & $>23.5$ & $>3.38$ \\
\hline ATHDF-S_J223317.5-605416A & 7.754 & $>23.5$ & $>3.71$ \\
\hline ATHDF-S_J223317.5-605416B & 5.570 & $>23.5$ & $>3.57$ \\
\hline ATHDF-S_J223403.1-605101 & 12.043 & $>23.5$ & $>3.90$ \\
\hline ATHDF-S_J223454.0-604904 & 0.483 & $>23.5$ & $>2.50$ \\
\hline ATHDF-S_J223527.8-604639B & 1.423 & $>23.5$ & $>2.97$ \\
\hline ATHDF-S_J223319.1-604348 & 1.085 & $>23.5$ & $>2.86$ \\
\hline ATHDF-S_J223427.3-604258 & 0.571 & $>23.5$ & $>2.58$ \\
\hline ATHDF-S_J223417.8-604009 & 1.502 & $>23.5$ & $>3.00$ \\
\hline ATHDF-S_J223429.9-603629 & 0.671 & $>23.5$ & $>2.65$ \\
\hline ATHDF-S_J223224.0-603537 & 1.259 & $>23.5$ & $>2.92$ \\
\hline ATHDF-S_J223258.5-603346 & 1.010 & $>23.5$ & $>2.82$ \\
\hline ATHDF-S_J223509.5-603257 & 0.980 & $>23.5$ & $>2.81$ \\
\hline ATHDF-S_J223537.7-603013 & 2.860 & $>23.5$ & $>3.28$ \\
\hline ATHDF-S_J223236.5-603000 & 1.507 & $>23.5$ & $>3.00$ \\
\hline ATHDF-S_J223355.5-602956 & 1.534 & $>23.5$ & $>3.01$ \\
\hline ATHDF-S_J223140.6-602924 & 4.962 & $>23.5$ & $>3.52$ \\
\hline ATHDF-S_J223117.4-602850 & 7.756 & $>23.5$ & $>3.71$ \\
\hline ATHDF-S_J223306.6-602425 & 1.949 & $>23.5$ & $>3.11$ \\
\hline ATHDF-S_J223410.2-602324 & 0.582 & $>23.5$ & $>2.58$ \\
\hline
\end{tabular}

We class sources as radio-loud AGNs if they have $R_{I}=$ $\log \left(S_{1.4 \mathrm{GHz}} / S_{I \text {-band }}\right)>2.5$. A source exceeding this ratio always lies above the radio-loud QSO track for redshifts 05 (Figure 21). Using this criterion, we identify 44 sources as radio-loud AGNs, including the sources with only limits in $I$ magnitude. These sources are listed in Table 12.

One interesting source classified as radio loud is ATHDFS_J223343.7-603651 which has a $1.4 \mathrm{GHz}$ flux density of $0.070 \mathrm{mJy}$. This faint source is at the detection limit of the CTIO images, and we have no redshift information for it. In Paper III we quote a radio spectral index of $\alpha_{1.4 \mathrm{GHz}}^{2.5 \mathrm{GHz}}=-1.14 \pm 0.39$ for this source and identified it as an ultra-steep spectrum radio source. The radio-loud classification of this source is consistent with it being a high redshift radio galaxy.

The distribution of the radio-to-optical ratio of the $I$-bandidentified sources is shown in Figure 22. The $R_{I}$ distribution of sources brighter than $1 \mathrm{mJy}$ shows signs of bimodality, in accordance with Ivezić et al. (2002). Although the small number of sources makes it difficult to accurately determine the shape of the radio-to-optical ratio distribution, we find the number of radio bright sources peaks at $R_{I}>2$ and has a dip at $0<R_{I}<1$. If we then assume that most of the optically selected AGNs in the HDF-S are not detected in the radio, then these undetected optical AGNs have $R_{I}<0$ and the unobserved part of the $R_{I}$ distribution must rise for $R_{I}<0$. This is inconsistent with White et al. (2000), but this previous work only had shallow imaging ( $I \sim 18$ th mag compared to our $I=23.5$ limit). Our sample is radio selected, however, so a complete radio survey of a sample of optically selected AGNs is needed to confirm the radio-loud/radio-quiet bimodality of the AGNs. Sixty percent $(31 / 51)$ of these radio bright sources have ratios greater than $R_{I}>2.5$, meeting our conservative radio-loud criterion.

For the full sample, the radio-to-optical ratio shows no sign of bimodality and has a roughly Gaussian distribution with a peak at $R_{I}=0.5-1$ (Figure 22). However, we only have $I$-band detections for $66 \%$ of the sources, and deeper optical imaging is needed to determine the distribution at $R_{I}>2$. The faint radio sources are more radio quiet than bright radio sources (as expected). The $R_{I}$ distribution for the fainter radio sources also shows an extended tail at high ratios, which means we cannot rule out low-luminosity AGNs in this population. We posit that there may be a lack of sources with ratios in the region $0<R_{I}<1$ at higher radio flux densities, but starbursts and radio-intermediate AGNs increase in number as fainter radio flux densities are probed, so the gap in the $R_{I}$ distribution between 0 and 1 is filled (see Figure 23). We also note that there is a subsample of radio sources which are undetected in the $I$-band with high radio-to-optical ratios $\left(R_{I}>1.5\right)$, which are the optically faint microjansky sources which are discussed in Section 2.3.3. Their ratios imply that they are radio-loud AGNs or starbursts with large dust obscuration.

\subsection{Radio Power and Redshift Distribution}

Radio luminosities for the ATHDF-S sources are calculated with the redshifts presented in this paper. In Figure 24, we plot the $1.4 \mathrm{GHz}$ radio luminosity of the ATHDF-S radio sources against redshift for the spectroscopic and photometric redshift samples. The galaxies classed as star-forming from $2 \mathrm{dF}$ spectra have luminosities in the range $\log \left(P_{1.4 \mathrm{GHz}}\right) \sim 20.5-23.5 \mathrm{~W}$ $\mathrm{Hz}^{-1}$. The early-type galaxies have larger radio powers of $\log \left(P_{1.4 \mathrm{GHz}}\right) \sim 21.5-24.5 \mathrm{~W} \mathrm{~Hz}^{-1}$. This is expected as the radio emission from early-type galaxies is driven by a weak AGN, which is more radio powerful than star-formation processes. Also as expected, the broadline AGNs are the most powerful radio sources $\left(\log \left(P_{1.4 \mathrm{GHz}}\right) \sim 23-26 \mathrm{~W} \mathrm{~Hz}^{-1}\right)$. Consistent with Smolčić et al. (2008), we find significant overlap in the radio powers of the star-forming and early galaxy types, and even broadline AGNs can have similar radio powers to the most powerful starbursts. This means that radio power alone cannot be used as a discriminator of source types.

The redshift distribution of our sources with spectroscopic redshifts is shown in Figure 25. The median redshift of the whole spectroscopic sample is 0.34 . The star-forming galaxies are at similar redshifts to the early-type galaxies, with median redshifts of 0.28 and 0.31 for the star-forming and early-type samples, respectively. We note that there is a lack of both spectroscopic star-forming and early-type galaxies at $z>0.5$ because of our survey sensitivity limit. The solid line in Figure 24, which marks the radio power sensitivity of our survey, 

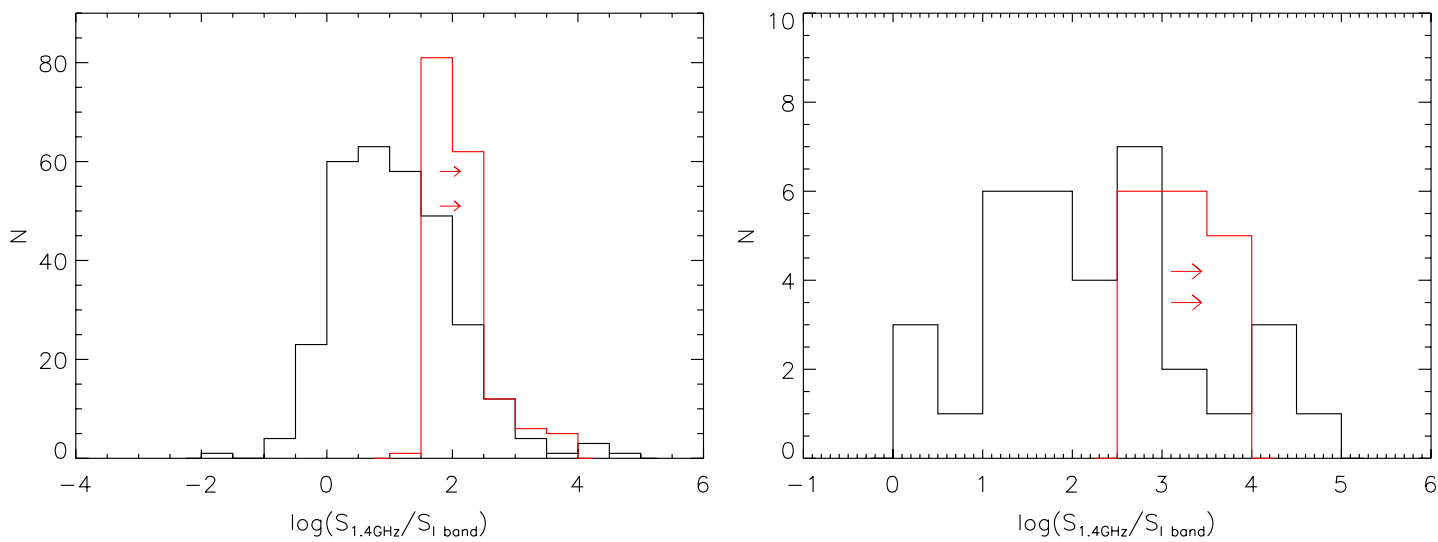

Figure 22. Radio-to-optical ratio $\left(R_{I}\right)$ distribution of the ATHDF-S sources with CTIO $I$-band counterparts (black solid line). The lower limit of $R_{I}$ for sources not detected in the $I$-band are determined using the nominal CTIO $I$-band limit of 23.5 mag. These lower limits are shown as the red histogram. Left: full ATHDF-S sample. Right: radio bright $\left(S_{1.4 \mathrm{GHz}}>1 \mathrm{mJy}\right)$ sample.

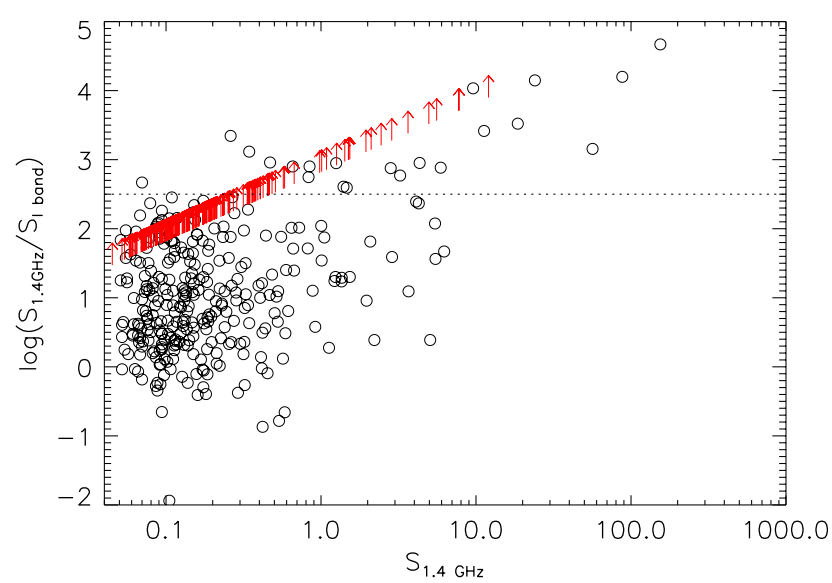

Figure 23. Radio-to-optical ratio $\left(R_{I}\right)$ plotted against $1.4 \mathrm{GHz}$ flux density. The dotted line marks our radio-loud criterion of $R_{I}>2.5$. The bright $\left(S_{1.4 \mathrm{GHz}}>\right.$ $1 \mathrm{mJy}$ ) radio sources have higher ratios than the faint sub-mJy radio sources, which have intermediate radio-to-optical ratios of $0<R_{I}<1$. There is sizeable number of radio sources at the $0.1 \mathrm{mJy}$ level with $R_{I} \gtrsim 2$, as indicated by the radio sources not detected in the $I$-band imaging (arrows), and some of these may be radio-loud AGNs.

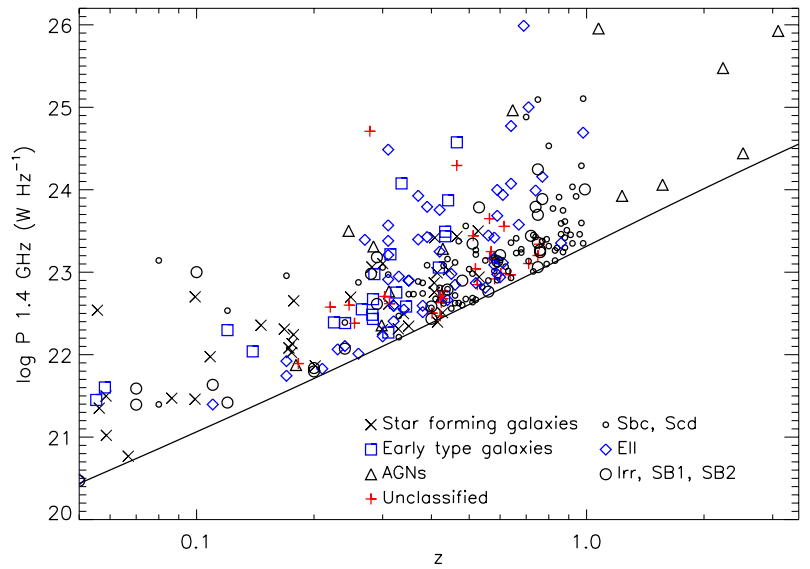

Figure 24. $1.4 \mathrm{GHz}$ luminosity $\left(\mathrm{W} \mathrm{Hz}^{-1}\right)$ of all our radio sources that have redshifts, as a function of redshift. The symbols are as shown in the legend. The left column of the legend is for sources with $2 \mathrm{dF}$ spectroscopic redshifts and the right column for sources with photometric redshifts. The solid line marks the detection limit of $0.050 \mathrm{mJy}$.

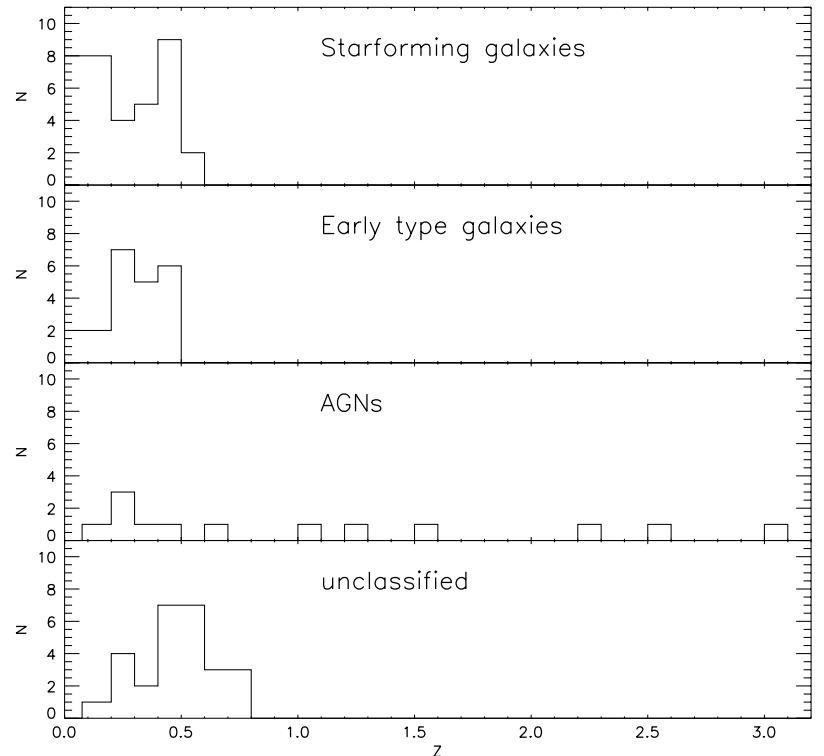

Figure 25. Redshift distribution of our radio sources with $2 \mathrm{dF}$ spectra. Different spectral classes are drawn separately. From top to bottom: star-forming galaxies, early-type galaxies, AGNs, and unclassified. See the text for description of classes.

shows how our radio survey becomes increasingly insensitive to sources with star-forming and early-type radio powers as higher redshifts are probed. The broadline AGNs are detected at both low and moderate redshifts, but only the most powerful $\left(\log \left(P_{1.4 \mathrm{GHz}}\right) \gtrsim 24\right)$ are detected at $z>1$. The highest redshift source is a broadline AGN estimated to be at $z=3.1$. Finally we note that the unclassified sources are at greater redshifts than the star-forming or early-type galaxies, with a median redshift of 0.46 . The greater distance to these sources make them on average fainter in the optical, and thus the spectroscopy of these sources is of lower quality, as expected.

In Figure 26 we show the photometric redshift distribution of our sources, sorted into best-fit template types. The late type galaxies, Sbc and Scd, show a Gaussian-like distribution with a peak at $z=0.35$ and an extended tail. The distribution of the early-type galaxies is similar, with a peak also at $z=0.35$. Again we argue that the decline in the number of sources in these two samples for $z>0.5$ is probably because of our survey 


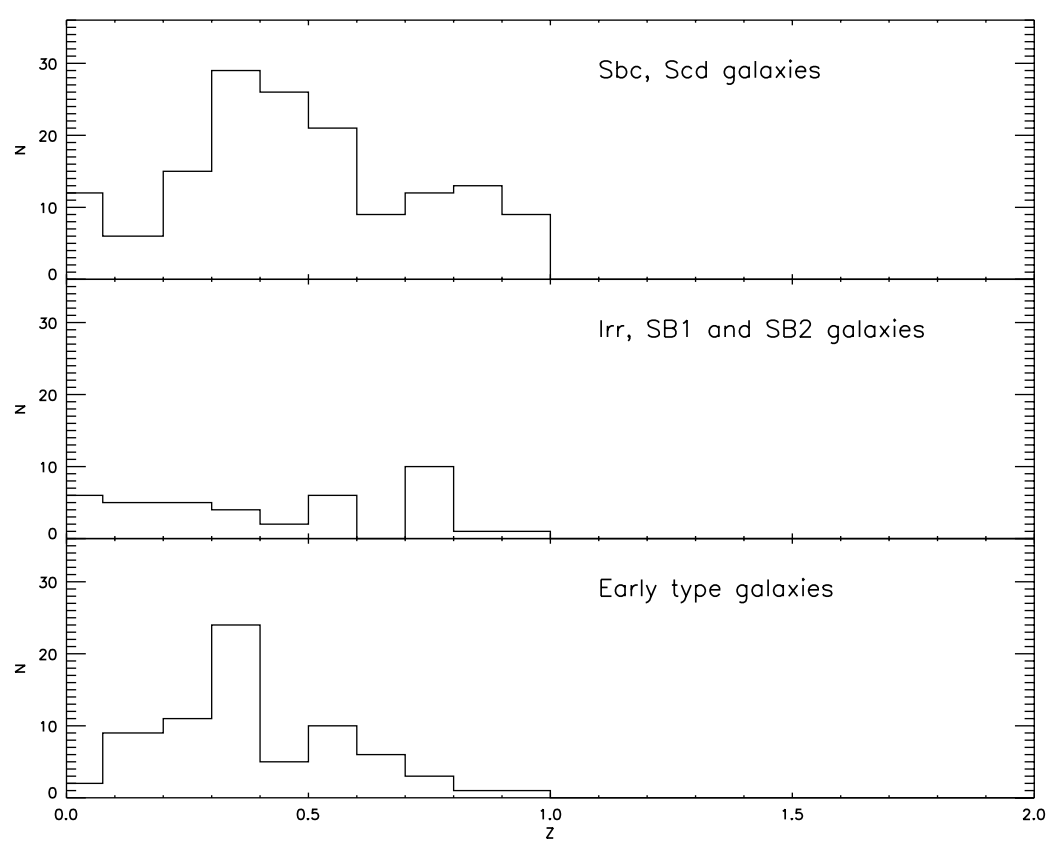

Figure 26. Redshift distribution of our radio sources with photometric redshifts. Samples with different best-fit templates are drawn separately. From top to bottom: late-type galaxies (Sbc and Scd), starbursts/irregulars, and early-type galaxies. See the text for description of classes.

sensitivity. The starbursts and irregulars appear to have a more uniform distribution than the early- or late-type samples.

\section{SUMMARY}

We have cross-matched the ATHDF-S radio sources with optical imaging from CTIO. We find 306/465 (66\%) of the radio sources which lie within the region covered by the CTIO imaging have counterparts to $I=23.5$.

The HST imaging was also used to search for optical counterparts to our radio sources. The HST imaging reaches a sensitivity approximately $2.5 \mathrm{mag}$ deeper than the CTIO imaging. This identifies a further $\sim 12 \%$ of sources in the HST region. Thus, the identification rate of sub-mJy radio sources to $\mathrm{I}=26.0$ is estimated to be $79 \%$. A sizable proportion ( $20 \%$ ) are fainter than this and remain optically unidentified. These optically faint microjansky sources, also found in other radio surveys, are thought to be a mix of starbursts at $1<z<3$ and high redshift obscured AGNs.

We have presented spectroscopic redshifts derived from $2 \mathrm{dF}$ spectra for 98 of the radio sources. Where the spectra is of sufficient quality, the sources have been classified as early-type, star-forming, Seyfert, or broadline AGNs. The spectroscopic sample consists of $22 \%$ early type, $37 \%$ late type, and $13 \%$ Seyferts or broadline AGNs. The median redshift of the sample is $z=0.34$ and a broadline $\mathrm{AGN}$ at $z=3.1$ has been discovered. Photometric redshifts from five-band CTIO photometry have also been calculated for 264 ATHDF-S sources. A total of $56 \%$ (266/473) of the radio sources have redshift information.

Using the available optical information, our main results are as follows:

The observed $I$-band magnitude of $S_{1.4 \mathrm{GHz}}>0.5 \mathrm{mJy}$ radio sources is $0.8 \mathrm{mag}$ brighter than the faintest radio sources $\left(S_{1.4 \mathrm{GHz}}<0.1 \mathrm{mJy}\right)$.

The median restframe $B-I$ color of the optical hosts is consistent with that of a Sbc galaxy.
There is little variation in the restframe optical colors of the host galaxies with redshift (to $z \sim 1$ ) or $1.4 \mathrm{GHz}$ flux density. This suggests that faint and bright radio sources have similar host galaxies and that the host galaxies do not evolve much with redshift. It is also further evidence that there is a significant fraction of low-luminosity or radioquiet AGNs in the sub-mJy radio population.

Using a conservative radio-to-optical flux density ratio, we conclude that 44 of the sources are radio loud and most likely powered by an AGN. We find that $61 \%$ (31/51) of bright radio sources $\left(S_{1.4 \mathrm{GHz}}>1 \mathrm{mJy}\right)$ have a radio-tooptical ratio that fits the radio-loud criterion.

The distribution of the radio-to-optical ratios of the bright $\left(S_{1.4 \mathrm{GHz}}\right)$ radio sources is consistent with a radioloud/radio-quiet bimodality. However, complete radio observations of an optically selected sample of AGNs are required to confirm the dichotomy. We find no evidence for the radio-loud/radio-quiet bimodality in the full ATHDF-S sample, which we believe is due to radiointermediate starbursts, and low-luminosity AGNs becoming more numerous at sub-mJy flux density levels.

The mid-IR wavelengths can be good discriminators of starformation versus AGN emission (Lacy et al. 2004; Stern et al. 2005). The HDF-S has been observed in the IR by the Spitzer Space Telescope. Future papers in this series will present the Spitzer identifications of the ATHDF-S radio sources to further investigate the nature of faint radio sources.

M.T.H. would like to thank Nick Seymour for useful discussions. A.F.S. acknowledges support from MEC Project AYA2006-14056 and a "Ramón y Cajal" research contract. The Australia Telescope Compact Array is part of the Australia Telescope, which is funded by the Commonwealth of Australia for operation as a National Facility managed by the CSIRO. 


\section{REFERENCES}

Afonso, J., Mobasher, B., Koekemoer, A., Norris, R. P., \& Cram, L. 2006, AJ, 131,1216

Alexander, D. M., Brandt, W. N., Hornschemeier, A. E., Garmire, G. P., Schneider, D. P., Bauer, F. E., \& Griffiths, R. E. 2001, AJ, 122, 2156

Barger, A. J., Cowie, L. L., \& Richards, E. A. 2000, AJ, 119, 2092

Barger, A. J., Cowie, L. L., \& Wang, W.-H. 2007, ApJ, 654, 764

Bertin, E., \& Arnouts, S. 1996, A\&AS, 117, 393

Bruzual, A. G., \& Charlot, S. 1993, ApJ, 405, 538

Casertano, S., et al. 2000, AJ, 120, 2747

Chapman, S. C., Richards, E. A., Lewis, G. F., Wilson, G., \& Barger, A. J. 2001, ApJ, 548, L147

Chapman, S. C., et al. 2003, ApJ, 585, 57

Coleman, G. D., Wu, C.-C., \& Weedman, D. W. 1980, ApJS, 43, 393

Colless, M., et al. 2001, MNRAS, 328, 1039

Condon, J. J. 1984, ApJ, 287, 461

Condon, J. J. 1989, ApJ, 338, 13

Condon, J. J., Odell, S. L., Puschell, J. J., \& Stein, W. A. 1981, ApJ, 246, 624

Croton, D. J., et al. 2006, MNRAS, 365, 11

Elvis, M., et al. 1994, ApJS, 95, 1

Fernández-Soto, A., Lanzetta, K. M., \& Yahil, A. 1999, ApJ, 513, 34

Francis, P. J., Hewett, P. C., Foltz, C. B., Chaffee, F. H., Weymann, R. J., \& Morris, S. L. 1991, ApJ, 373, 465

Gardner, J. P., et al. 2000, AJ, 119, 486

Glazebrook, K., Verma, A., Boyle, B., Oliver, S., Mann, R. G., \& Monbleau, D. 2006, AJ, 131, 2383

Hawkins, M. R. S. 2002, MNRAS, 329, 76

Ho, L. C., \& Peng, C. Y. 2001, ApJ, 555, 650

Huynh, M. T., Jackson, C. A., \& Norris, R. P. 2007a, AJ, 133, 1331

Huynh, M. T., Jackson, C. A., Norris, R. P., \& Prandoni, I. 2005, AJ, 130, 1373

Huynh, M. T., Pope, A., Frayer, D. T., \& Scott, D. 2007b, ApJ, 659, 305

Ivezić, Ž., et al. 2002, AJ, 124, 2364

Jarvis, M. J., \& Rawlings, S. 2004, New Astron. Rev., 48, 1173

Kellermann, K. I., Sramek, R., Schmidt, M., Shaffer, D. B., \& Green, R. 1989, AJ, 98, 1195
Kewley, L. J., Heisler, C. A., Dopita, M. A., \& Lumsden, S. 2001, ApJS, 132, 37 Kinney, A. L., Calzetti, D., Bohlin, R. C., McQuade, K., Storchi-Bergmann, T., \& Schmitt, H. R. 1996, ApJ, 467, 38

Kormendy, J., \& Richstone, D. 1995, ARA\&A, 33, 581

Labbé, I., et al. 2003, AJ, 125, 1107

Lacy, M., et al. 2004, ApJS, 154, 166

Landolt, A. U. 1992, AJ, 104, 340

Lucas, R. A., et al. 2003, AJ, 125, 398

Muxlow, T. W. B., et al. 2005, MNRAS, 358, 1159

Norris, R. P., et al. 2005, AJ, 130, 1358

Palunas, P., et al. 2000, ApJ, 541, 61

Pope, A., et al. 2006, MNRAS, 370, 1185

Richards, E. A., Fomalont, E. B., Kellermann, K. I., Windhorst, R. A., Partridge, R. B., Cowie, L. L., \& Barger, A. J. 1999, ApJ, 526, L73

Rowan-Robinson, M., Benn, C. R., Lawrence, A., McMahon, R. G., \& Broadhurst, T. J. 1993, MNRAS, 263, 123

Rudnick, G., et al. 2001, AJ, 122, 2205

Sawicki, M., \& Mallén-Ornelas, G. 2003, AJ, 126, 1208

Sealey, K. M., Drinkwater, M. J., \& Webb, J. K. 1998, ApJ, 499, L135

Seymour, N., McHardy, I. M., \& Gunn, K. F. 2004, MNRAS, 352, 131

Seymour, N., et al. 2008, MNRAS, 386, 1695

Simpson, C., et al. 2006, MNRAS, 372, 741

Smolčić, V., et al. 2008, ApJ, in press (arXiv:0803.0997)

Sramek, R. A., \& Weedman, D. W. 1980, ApJ, 238, 435

Stern, D., et al. 2005, ApJ, 631, 163

Stocke, J. T., Morris, S. L., Weymann, R. J., \& Foltz, C. B. 1992, ApJ, 396, 487

Teplitz, H. I., Hill, R. S., Malumuth, E. M., Collins, N. R., Gardner, J. P., Palunas, P., \& Woodgate, B. E. 2001, ApJ, 548, 127

Vanzella, E., et al. 2002, A\&A, 396, 847

Wall, J. V., Benn, C. R., Grueff, G., \& Vigotti, M. 1986, Highlights in Astronomy, 7, 345

White, R. L., et al. 2000, ApJS, 126, 133

Williams, R. E., et al. 2000, AJ, 120, 2735

Windhorst, R. A., Miley, G. K., Owen, F. N., Kron, R. G., \& Koo, D. C. 1985, ApJ, 289, 494

Yahata, N., Lanzetta, K. M., Chen, H., Fernández-Soto, A., Pascarelle, S. M., Yahil, A., \& Puetter, R. C. 2000, ApJ, 538, 493 